Issued by Sandia National Laboratories, operated for the United States Department of Energy by Sandia Corporation.

NOTICE: This report was prepared as an account of work sponsored by an agency of the United States Government. Neither the United States Government, nor any agency thereof, nor any of their employees, nor any of their contractors, subcontractors, or their employees, make any warranty, express or implied, or assume any legal liability or responsibility for the accuracy, completeness, or usefulness of any information, apparatus, product, or process disclosed, or represent that its use would not infringe privately owned rights. Reference herein to any specific commercial product, process, or service by trade name, trademark, manufacturer, or otherwise, does not necessarily constitute or imply its endorsement, recommendation, or favoring by the United States Government, any agency thereof, or any of their contractors or subcontractors. The views and opinions expressed herein do not necessarily state or reflect those of the United States Government, any agency thereof, or any of their contractors.

Printed in the United States of America. This report has been reproduced directly from the best available copy.

Available to DOE and DOE contractors from

Office of Scientific and Technical Information

P.O. Box 62

Oak Ridge, TN 37831

Prices available from (703) 605-6000

Web site: http://www.ntis.gov/ordering.htm

Available to the public from

National Technical Information Service

U.S. Department of Commerce

5285 Port Royal Rd

Springfield, VA 22161

NTIS price codes

Printed copy: A05

Microfiche copy: A01

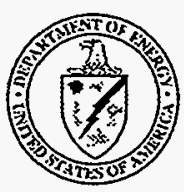




\section{DISCLAIMER}

Portions of this document may be illegible in electronic image products. Images are produced from the best available original document. 
SAND99-1175

Unlimited Release

Printed May 1999

\title{
Ultrahard Multilayer Coatings
}

\author{
T. A. Friedmann, N. Missert, and D. M. Follstaedt \\ Nanostructure and Semiconductor Physics Department \\ J.P. Sullivan \\ Advanced Materials and Device Sciences \\ J. A. Knapp \\ Radiation Solid Interactions and Processing \\ D. R. Tallant \\ Materials Characterization \\ P. P. Newcomer \\ Nanostructures and Advanced Materials Chemistry \\ M. Dugger \\ Materials Aging and Reliability Interfaces \\ Sandia National Laboratories \\ P.O. Box 5800 \\ Albuquerque, NM 87185 \\ D. L. Medlin \\ Microstructure Research \\ P. B. Mirkarimi, K. F. McCarty \\ Surface Chemistry \\ Sandia National Laboratories \\ P.O. Box 969 \\ Livermore, CA 94550 \\ Lawrence H. Friedman \\ Department of Physics \\ University of California \\ Berkeley CA 94720 \\ D.C. Chrzan \\ Department of Materials Science and Mineral Engineering \\ University of California \\ Berkeley CA
}

\begin{abstract}
We have developed a new multilayer a-tC material that is thick, stress-free, adherent, low friction, and with hardness and stiffness near that of diamond. The new a-tC material is deposited by pulsed-laser deposition (PLD) at room temperature, and fully stress-relieved by a short thermal anneal at $600^{\circ} \mathrm{C}$. A thick multilayer is built up by repeated deposition and annealing steps. We measured $88 \mathrm{GPa}$ hardness, $1100 \mathrm{GPa}$ Young's modulus, and 0.1 friction coefficient (under high load). Significantly, these results are all well within the range reported for crystalline diamond. In fact, this material, if considered separate from crystalline diamond, is the 2nd hardest material known to man. Stress-free a-tC also has important advantages over thin film diamond; namely, it is smooth, processed at lower temperature, and can be grown on a much broader range of substrates. This breakthrough will enable a host of applications that we are actively pursuing in MEMs, sensors, LIGA, etc.
\end{abstract}


We have developed a new cBN deposition process - ion-assisted sputter deposition - that produces $\mathrm{cBN}$ films with vastly improved mechanical properties. These $\mathrm{cBN}$ films have a columnar grain structure, have high hardness (higher than bulk $\mathrm{cBN}$ ), are smooth, and adhere well to $\mathrm{Si}$ substrates. Importantly, they can be grown to thicknesses approaching $1 \mu \mathrm{m}$ without delamination from the Si substrate, an increase of $10 \mathrm{x}$ in the practical thickness limit over previous deposition processes. Also, we grew films of TiN and $\mathrm{B} 4 \mathrm{C}$ that are as hard as bulk samples. We have grown bilayer samples of $\mathrm{B} 4 \mathrm{C} / \mathrm{cBN}$ using the new ion-assisted sputter process. These bilayer samples are hard, adhere well to the substrate, and have smooth interfaces. We have also grown some large period TiN/cBN multilayers (repeating layers of thick $\mathrm{cBN}$ on top of thin TiN) that adhere well to Si. We used finite element modeling techniques to extract yield and elastic stresses from nanoindentation curves of these thin films. We have calculated the stress/strain field due to a dislocation in a multilayer film coating.

In addition, we have dramatically improved our $\mathrm{CBN}$ films grown by ion-assisted PLD (or IAPLD). We combined $x$-ray reflectivity, FTIR and TEM characterization to optimize the IAPLD process to obtain films that have the bulk density of $\mathrm{cBN}$, a columnar $\mathrm{cBN}$ grain structure with greatly reduced $\mathrm{hBN}$ inclusions, and smooth surfaces. We have used in situ monitoring of the stress buildup during growth combined with subsequent x-ray reflectivity and FTIR analysis to map the conditions under which high quality $\mathrm{cBN}$ films are formed. Significantly, we find that the growth stress decreases with increasing growth temperature. Unfortunately, this is accompanied by an increase in the thickness of the soft $\mathrm{hBN}$ nucleation layer, preventing the synthesis of practical multilayers of cBN/a-tC.

Also, we have vastly improved our finite element modeling techniques to extract yield, elastic stresses, and hardness from nanoindentation curves. We used finite-element modeling of the experimental nanoindentation curves to separate the "intrinsic" film response from the measured substrate/film response for a series of a-tC samples grown under identical conditions from 400 to $10000 \AA$ thick. The modeling gave the same result for each layer independent of thickness. These modeling results have implications for indentation testing of all very hard materials, both thin layers and bulk. For very hard layers on softer materials, the modeling has shown that sink-in is a severe problem. Furthermore, the simulations reveal significant deformation of the indenter tip. Since a Sneddon-based analysis of indentation load-displacement data uses the assumption of a rigid indenter, the significant deformations we calculate appear to be a fundamental problem. Finiteelement modeling appears to be the only way to accurately deduce the hardness and modulus of a material that approaches the hardness of diamond.

Our theoretical studies have focused on the role that sources play in the formation of pileups. Early on, it was determined that the problem of sources had not been treated adequately within the context of a grain size effect in a pure material. Since this is the logical starting point for further theory development, we began by including source information into the continuum theory of pileup formation. This problem, which we solved exactly, gives an expression for the strength of a material with a given grain size. To investigate the accuracy of the continuum approximation, we compare the results of the continuum theory with exact numerical results for a discrete dislocation model. The agreement between the continuum theory and the discrete dislocation model is excellent, implying that the continuum theory is a good description of pileup formation in systems with more than a few dislocations. 


\section{Contents}

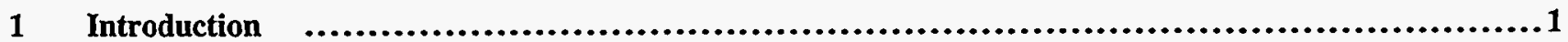

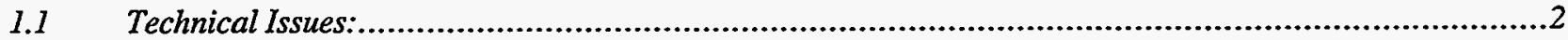

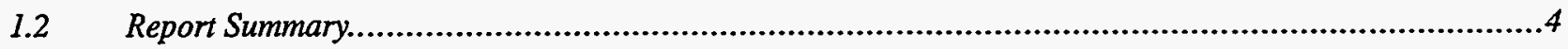

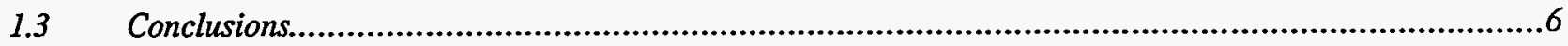

2 Growth, characterization, and stress relaxation of amorphous tetrahedral carbon films

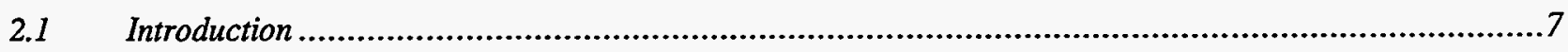

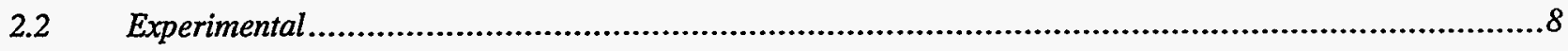

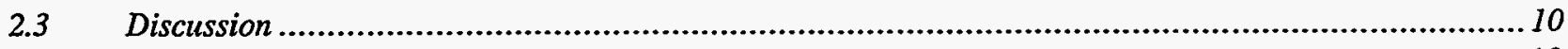

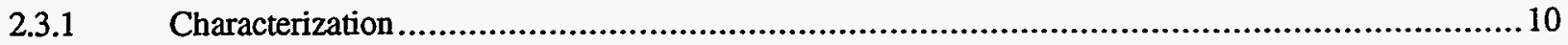

2.3.2 Thermal Annealing of a-tC films grown at high fluence ...................................................15

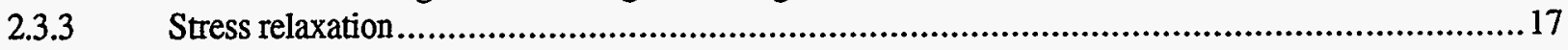

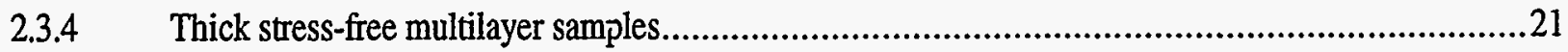

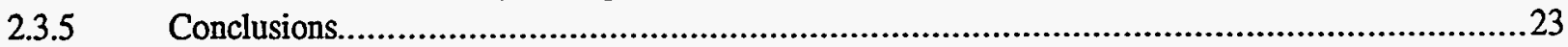

2.4 Finite-element modeling of nanoindentation for evaluating mechanical properties of the amorphoustetrahedral carbon films .............................................................................................................................2 24

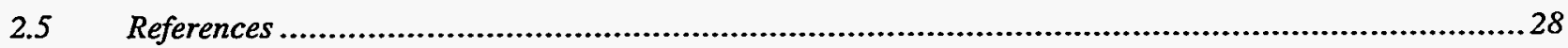

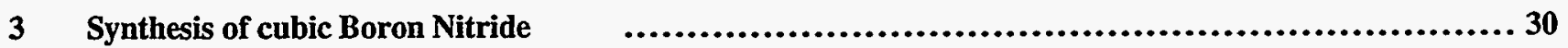

3.1 Thick cubic boron nitride produced by a hybrid ion assisted sputtering technique ...............................30

3.2 Introduction

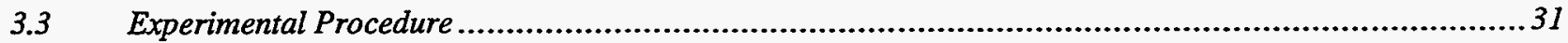

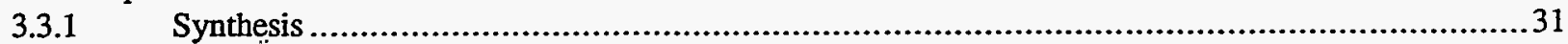

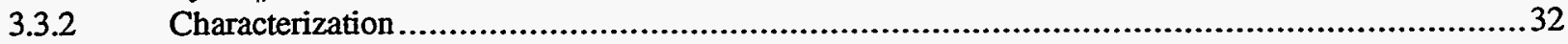

3.4 Compositional and microstructural analysis ............................................................................3

3.4.1 Measuring the mechanical properties of hard ceramics by nanoindentation ...............................36

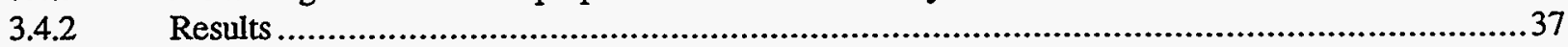

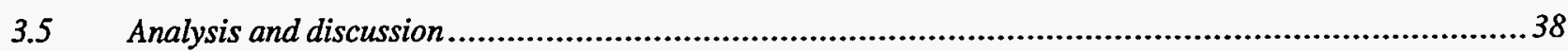

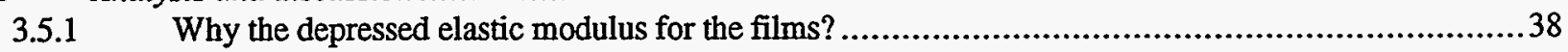

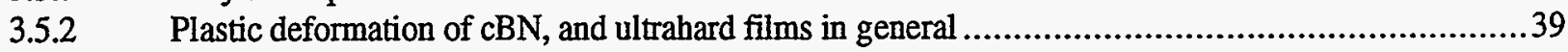

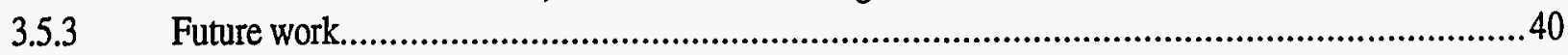

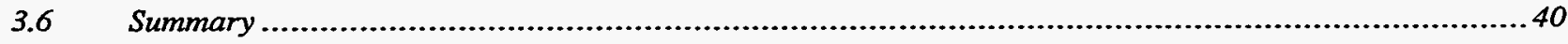

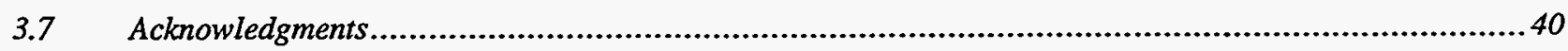

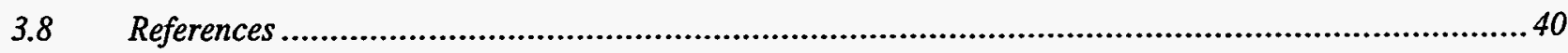

4 Growth and characterization of BN thin films by pulsed laser deposition - application of

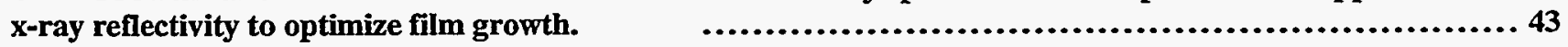

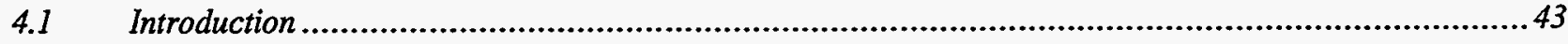

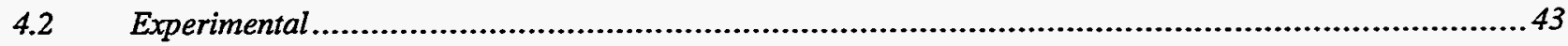

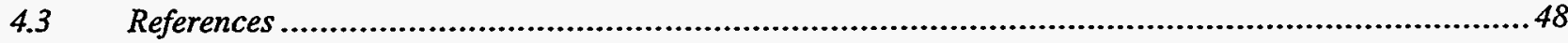




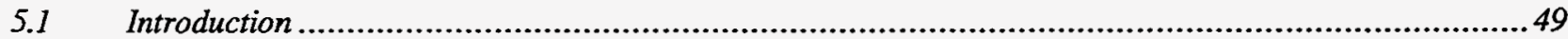

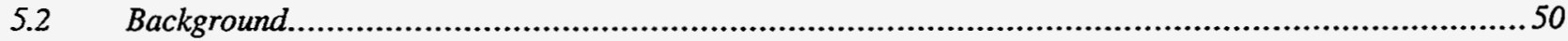

$5.3 \quad$ Alternative derivation of the Hall-Petch relation ......................................................................51

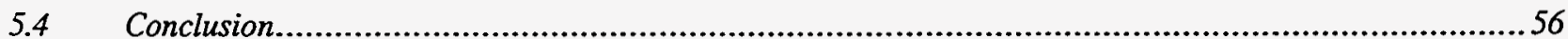

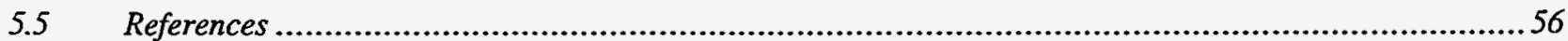

6 Continuum analysis of dislocation pileups - influence of sources $\quad$.......................... 57

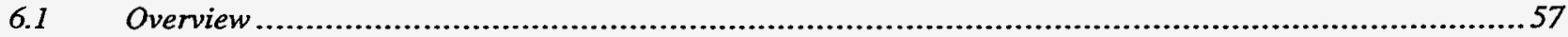

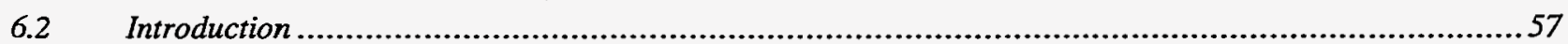

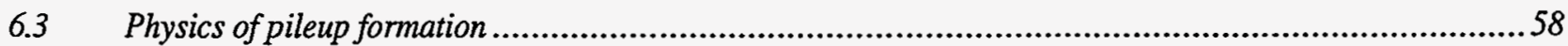

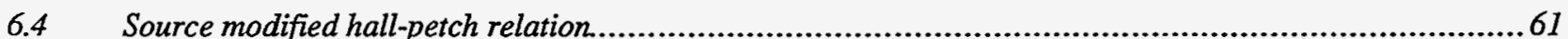

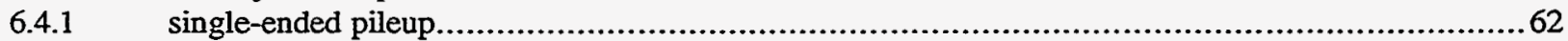

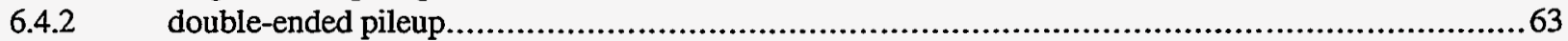

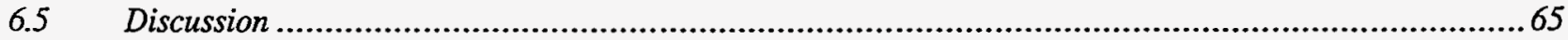

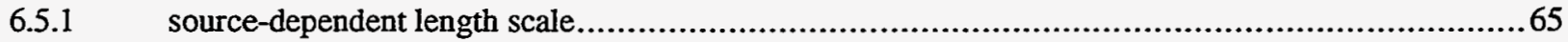

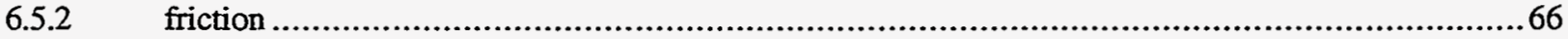

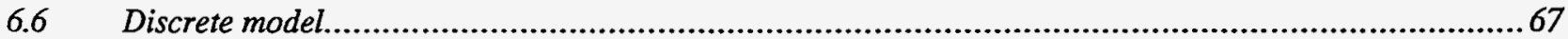

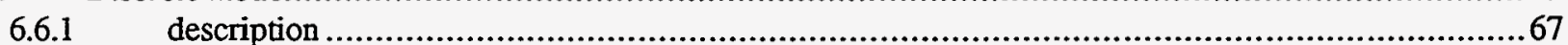

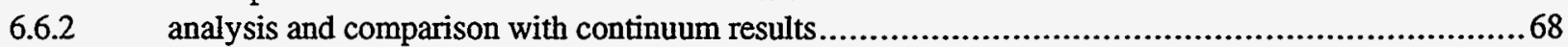

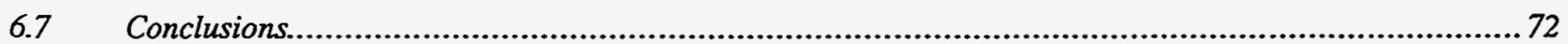

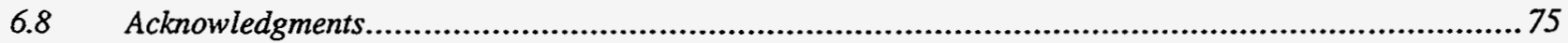

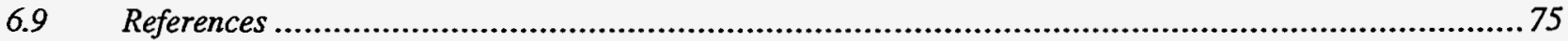

6.10 Appendix a: mathematical appendix

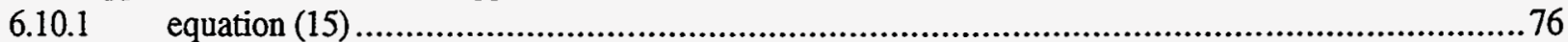

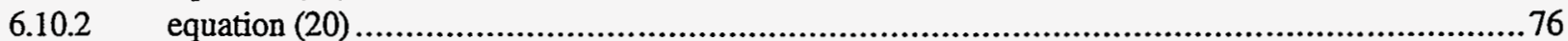

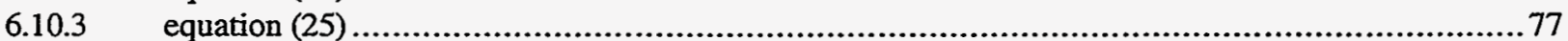




\section{Ultrahard Multilayer Coatings}

\section{Introduction}

THE PURPOSE OF THIS PROJECT WAS TO EXPLORE THE PRODUCTION OF CERAMIC MULTLAYER STRUCTURES THAT COULD POTENTIALLY BE HARDER THAN ANY NATURAL OR ARTIFICIAL MATERIAL. Diamond and cubic boron nitride ( $\mathrm{CBN}$ ) are the two hardest substances known to man. Numerous proven technologies rely on the superior mechanical properties of these materials. The question arose: Was it possible to manufacture a material that is harder than diamond? In theory, the answer was yes. Experiment indicates that properly grown multilayer coatings of two materials are harder than either of the materials making up the individual layers. The increase in hardness is mainly due to the resistance of dislocation flow across the interfaces between phases of different elasticity. This experimental fact leads to the possibility that a new class of ultrahard materials - harder than diamond -- can be made by growing the appropriate multilayer film.

The proposed multilayers consisted primarily of hard, wide-band-gap semiconductors to form ultra-hard structures with possible hardnesses surpassing that of diamond. For this project, we defined multilayers as structures consisting of thin-film layers of materials. It is an established experimental fact that properly grown multilayer structures have yield strengths and hardnesses much greater than either of the constitutive phases. Hardness enhancements occur both in polycrystalline and single-crystal multilayers of metals and ceramics. This work is a combination of growth and analysis capabilities at Sandia combined with theoretical work done at the University of California at Berkeley.

The growth of multilayer films offers other tribological advantages besides hardness: control of the combined stress state of the composite system by tailoring the stress in the individual layers, increased fracture toughness due to crack deflection along the interfaces, and improved adhesion by producing a graded interfacial layer. We wanted to engineer the mechanical properties of these (mainly) ceramic materials by controlled multilayer growth, similarly to the process of engineering electronic properties of semiconductors by incorporating them into multilayer structures.

To date, there has been no success in growing multilayers of the hardest known materials (e.g., diamond and $\mathrm{cBN}$ ) due to the difficulty of growing these phases in suitable thin film form. At the beginning of this project, the project participants had developed much understanding of amorphous-tetrahedral carbon (a-tC), and $\mathrm{cBN}$ synthesis. The real problems associated with incorporating these materials into multilayers were understood, and much of the project was focused on improving the materials properties of the individual materials with the ultimate goal to incorporate them into multilayers. The significant problems were control of stress and adhesion in both materials systems, improvement in film crystallinity in the cBN system, and compatibility between the two systems. We can say that this effort has produced several exciting advances regarding the materials properties of the individual materials. These results will be detailed in the following report. These improvements proved quite exciting but we were unable to successfully make multilayers with $\mathrm{cBN}$ and a-tC components. However, the discoveries made on this project form the basis for ongoing work regarding applications of stress-free a-tC in sensors and micro-electromechanical machines (MEMS). Additionally, we have made a major contribution to the analysis of experimental nanoindentation results for hard films on soft substrates, that has the potential of becoming a standard analysis technique to improve the utility of that technique.

Our theoretical studies have focused on the role that sources play in the formation of pileups. Early on, it was determined that the problem of sources had not been treated adequately within the context of a grain size effect in a pure material. Since this is the logical starting point for further theory development, we began by including source information into the continuum theory of pileup formation. This problem, which we solved exactly, gives an expression for the 
strength of a material with a given grain size. To investigate the accuracy of the continuum approximation, we compare the results of the continuum theory with exact numerical results for a discrete dislocation model. The agreement between the continuum theory and the discrete dislocation model is excellent, implying that the continuum theory is a good description of pileup formation in systems with more than a few dislocations.

\subsection{Technical Issues:}

Hardness enhancements in multilayer structures are primarily attributed to the mechanism of dislocation-line-energy strengthening, as proposed by Koehler ${ }^{1,2}$. Simply put (fig. 1-1), in an $\mathrm{A} / \mathrm{B}$ multilayer in which the $\mathrm{B}$ layers possess a lower dislocation line energy than the A layers, a large external stress is needed to drive a dislocation from the B layers into the A layers. Since the flow of dislocations is inhibited, the artificial structure has an enhanced hardness (yield strength). The predicted enhancement is proportional to ( $\left.G_{A}-G_{B}\right) G_{B}$, where $G_{i}$ is the shear modulus of the "ith-type layer." The Koehler theory does not account for either dislocation interactions, slip transmission across interfaces, or other deformation mechanisms. Also, experiment indicates that this simple approach tends to underestimate the actual hardness enhancement found in crystalline multilayers.

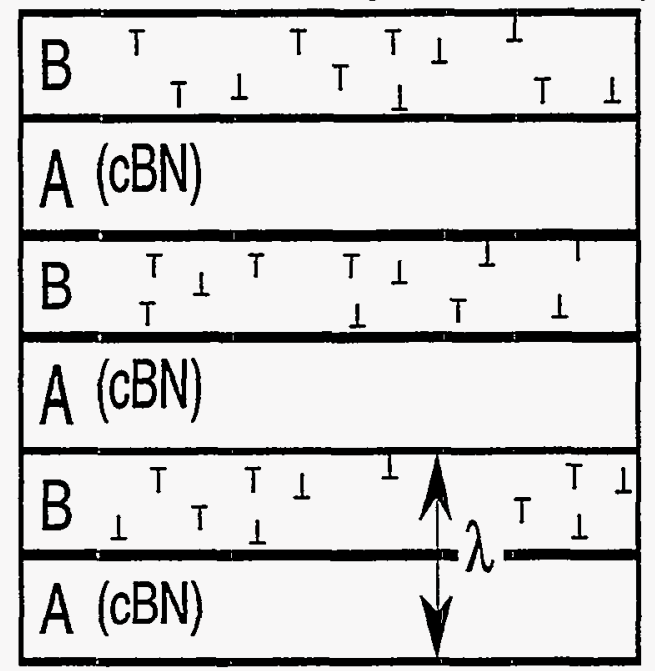

Fig. 1-1 Schematic illustration of multilayer consisting of alternating layers of 2 phases. The spatial periodicity $\lambda$ is typically 10 's to 100 's of atomic layers. Dislocations, as illustrated by the symbol $T$, occur preferentially in the softer $B$ phase due to energetic considerations. The hardness enhancement results from the large external stress needed to drive the dislocations from the $B$ phase to the $A$ phase.

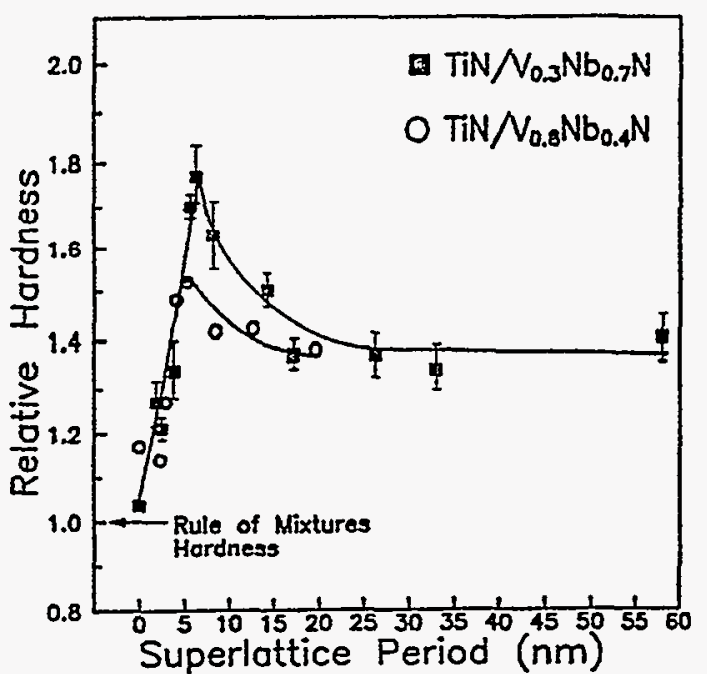

Fig. 1-2. Normalized hardness of $T$ iN/ $/ \mathrm{V}_{\mathrm{X}} \mathrm{Nb} 1-\mathrm{x} \mathrm{N}$ multilayers versus multilayer period. The maximum enhancement is about $75 \%$, producing a hardness of $\approx 3500 \mathrm{~kg} / \mathrm{mm} 2$, versus 2100,1560 , and $1400 \mathrm{~kg} / \mathrm{mm} 2$ for TiN, $\mathrm{VN}$, and $\mathrm{NbN}$, respectively. (P. B. Mirkarimi et al., J. Mater. Res. 9, 1456 (1994))

Theoretically, the ultimate hardness occurs in a multilayer grown with crystalline diamond as the " $\mathrm{A}$ " layer. Unfortunately, diamond-synthesis techniques produce continuous films only for thicknesses well above 1 micron. In such thick layers, interlayer effects (not interfaces) dominate the mechanical properties leading to minimal hardness enhancement. Thus, diamond-based multilayers with enhanced properties are not feasible. Fortunately, the same problem does not plague the other compounds considered (cBN, a-tC, TiN, and $\mathrm{B}_{4} \mathrm{C}$ ).

The authors had developed pulsed laser deposition (PLD), to grow single-layer films of $\mathrm{cBN}$, a-tC, and TiN. Importantly, these materials all have high hardness and are potential candidates for inclusion in an ultrahard multilayer coating (see table). In particular, we had 
detailed understanding of $\mathrm{CBN}$ (the 2nd hardest material next to diamond) synthesis using ionassisted PLD (IAPLD) and could routinely grow thin films (100's of $\AA$ ) with high phase purity. These films have a very fine-grained structure (grains 10 's of $\AA$ ).

Early on, we realized that we needed to fully characterize the individual materials and

\begin{tabular}{|c|c|c|}
\hline Material & $\begin{array}{c}\mathrm{C} 44 \tilde{\sim} \mathrm{G} \\
(\mathrm{GPa})\end{array}$ & $\begin{array}{c}\text { Hardness } \mathrm{H}_{\mathrm{V}} \\
\left(\mathrm{kg} / \mathrm{mm}^{2}\right)\end{array}$ \\
\hline diamond & 500 & 8000 \\
\hline $\mathrm{cBN}$ & 450 & 5000 \\
\hline B-SiC & 233 & 2600 \\
\hline TiN & 163 & 2100 \\
\hline DLC & $?$ & 7100 \\
\hline
\end{tabular}
optimize the deposition process for use in multilayers. We foresaw at least three major problems that needed to be solved before these materials could be incorporated into multilayers.

First, in the cBN and a-tC system, the films grow with a high degree of internal compressive stress (6-12 GPa). These stresses limit the utility of each individual system, since the films tend to spall from the substrate at thicknesses near 0.1-0.2 $\mu \mathrm{m}$. In addition, this would limit the utility of multilayers incorporating these materials since the stress build up in the multilayer would also limit the maximum achievable thickness. As will be detailed in this report, we have made tremendous progress in understanding and controlling stress in the individual systems, that has led to significant scientific and technical breakthroughs.

Second, the crystallinity of our cBN films needed improvement. The high ion energies used in the pulsed-laser deposition process were thought to be responsible for producing films with small grain size that proved to be quite soft. We solved this problem through further development of two different deposition processes. The first was the development of a new high-temperature hybrid electron-cyclotron resonance (ECR) sputter deposition process that led to the development of $\mathrm{cBN}$ films with vastly improved mechanical properties as measured with nanoindentation. These films were adherent and could be grown to thicknesses 10x greater than previously achieved due to the higher growth temperature. In addition, this process was compatible with multilayer development and could possibly be used to grow cBN/B ${ }_{4} \mathrm{C}$ multilayers. The second method was to improve the ion-assisted PLD process by going to lower ion energy, higher laser fluence, and higher growth temperature. We found that the film crystallinity was vastly improved by this technique. In addition; we proved the utility of x-ray reflectivity as a nondestructive characterization tool for $\mathrm{cBN}$ thin films. Measurements of stress buildup during growth showed that stress could be reduced (but not eliminated) by raising the substrate temperature.

Third, in cBN growth on Si substrates, we initially found that an interfacial layer of hexagonal $\mathrm{BN}$, the graphite-like phase, forms before $\mathrm{cBN}$. Given that $\mathrm{hBN}$ is relatively soft, its presence in appreciable quantities will degrade film properties. It was hoped that the use of an appropriate second layer (e.g. SiC) for the multilayer would eliminate this interfacial layer, but subsequent experiments did not show this to be the case. In addition, the best $\mathrm{cBN}$ films could be grown at temperatures above $1000{ }^{\circ} \mathrm{C}$. Unfortunately, the thickness of the soft hBN interfacial layer also increased with growth temperature. This $\mathrm{hBN}$ interfacial layer remains the single largest barrier to incorporating $\mathrm{cBN}$ into multilayer structures.

The problem is further complicated when one tries to make a-tC/cBN multilayers due to growth incompatibility in the synthesis requirements for the two phases. The highest fraction of $\mathrm{sp} 3$ bonding in DLC occurs in films grown at room temperature. Films grown above $200^{\circ} \mathrm{C}$ are completely sp2-bonded. As determined by data taken for this project, the optimum temperature for $\mathrm{cBN}$ synthesis is above $1000^{\circ} \mathrm{C}$ where films with the lowest stress and best crystallinity are found. Prior to this project we knew that we could grow reasonable purity $\mathrm{cBN}$ films as low as $150{ }^{\circ} \mathrm{C}$, but efforts during the project to improve the crystalline quality of these films proved unsuccessful. This problem is mitigated by the fact that DLC films grown at room temperature are structurally stable up to $800^{\circ} \mathrm{C}$ in vacuum. Thus, technically it is possible to modulate the growth temperature to a more optimum value for both DLC and $\mathrm{cBN}$ synthesis, but practical growth of multilayers under these conditions is not possible due to the long cycle times needed to cool down to room temperature after $\mathrm{cBN}$ growth. In addition, the thicker interfacial hBN layer 
produced at high temperature limits the feasibility of this approach since this thick hBN layer would produce soft multilayer materials.

\subsection{Report Summary}

Chapter 2 details the results of the project related to the deposition, characterization, and uses of a-tC deposited by pulsed laser deposition. In its simplest form, a pulsed laser is focused onto a graphite target in a vacuum chamber. A small volume of the target is vaporized with each pulse, and the resultant vapor expands adiabatically into the vacuum and condenses on a substrate placed to intercept the forward directed vaporization plume. The film properties are determined by the laser fluence on the target (fluence is simply the energy density of the laser spot on the target usually quoted in $\mathrm{J} / \mathrm{cm} 2$ ). The laser energy density in turn controls the energetics of the ablated species. For low fluences the ablated species are low in energy and the resultant films are graphitic in nature (soft, low density, opaque, and conductive). For high fluences the ablated species from the target are high in energy and the deposited films are more diamond-like (hard, high density, stiff, transparent, and electrically resistive). Unfortunately, the residual stress in the films also scales with the laser fluence, increasing to values near 7-8 Gpa for the most diamond-like films. The high compressive stress appears to be intrinsic to the growth process, in that all a-tC films grow with a high degree of compressive stress.

We have been able to completely relief the stress in a-tC films by thermal annealing. This was the first demonstration world-wide of this capability. (In fact, we still remain the only group to demonstrate stress free a-tC films by any method.) The stress in these films can be tailored from highly compressive to slightly tensile by precisely controlling the annealing time and temperature. By combining experimental nanoindentation and finite element modeling, we have shown that the resultant films are quite hard $(\mathrm{H}=88 \mathrm{GPa})$ and stiff $(\mathrm{Y}=1000 \mathrm{GPa})$. These values are well within those quoted for crystalline diamond. When considered as a separate material from diamond, a-tC is the second hardest material known to man. In situ monitoring of the stress build up during growth and relief during annealing was used to gain further understanding of the stress relief mechanisms. The data were fit to a model that successfully predicts the stress relief over 6 decades in time and $600{ }^{\circ} \mathrm{C}$ in temperature. We have demonstrated that it is possible to deposit thick multilayers of a-tC materials by repeated deposition and annealing steps. . To date, we have deposited up to $10 \mu \mathrm{m}$ thick films. In principle, there is no theoretical limit to the attainable thickness. Sandia has applied for a patent on the stress-free a-tC material.

This breakthrough has enabled several applications of this material that were previously thought impossible. Stress free films can be made into free-standing membranes. Previously, the high stresses in these materials would have caused any membrane to buckle, crack, and destroy itself upon release from the substrate. We have demonstrated membranes as thin as 600 $\AA$ and over one inch in diameter that can be handled routinely in air. These membranes are of potential use in resonant sensors or for electron and x-ray transparent windows. We are actively pursuing these applications. A follow on LDRD has been funded that uses a-tC membranes in Flexural-Plate Wave (FPW) sensors. These sensors work by electrically and magnetically setting up a resonant plate wave in a membrane. Electronically, the resonant frequency of the membrane is monitored, and any disturbance to the membrane is detected as a change in the resonant frequency. These sensors are great strain and temperature gauges and can also be used to detect airborne chemicals by coating the membrane surface with a chemically sensitive polymer.

Stress-free a-tC has superb tribological performance under high wear conditions. The friction coefficient measured in air is as low as 0.1 , its surface is naturally hydrophobic, and it is chemically inert, resistant to most wet chemicals. This makes stress-free a-tC ideal for application in MEMS devices. The current generation of MEMS technology relies on poly-Si as the building block material for the devices. MEMS although very small, are still machines, and 
are subject to failure due to wear. In fact, wear can be quite extreme in MEMS devices. Routinely poly-Si devices fail due to wear and this failure can occur in times as short as five minutes of operation. One report states that tribologically, diamond is 10,000 times better than poly-Si. That means that a-tC based MEMS ought to last 10,000 times longer than their poly-Si based counterparts. In addition, a-tC MEMS should not suffer from the same stiction problems reported in poly-Si. We are currently manufacturing a-tC based MEMS to test this prediction in follow-on work funded at Sandia by the LDRD department.

The end of chapter 2 details results of finite element modeling carried out to model nanoindentation results. It turns out that there are fundamental problems with using nanoindentation to assess the hardness and stiffness of very hard thin films on soft substrates. The major problem is "sink-in" in that the hard film tends to be pushed in to the substrate by the indenter tip and one measures a combined response of the film/substrate system and not the film properties. For our films, the results are further complicated by the fact that the films hardness is approaching that of the diamond indenter used to measure the hardness and the diamond tip can deform when pushed into the material. In the past, it was really only possible to compare nanoindentation results for these systems from one film to another, with no simple way to extract the intrinsic response of the film from the film/substrate combination. We have developed finiteelement modeling (FEM) to the point were accurate numbers for the yield, stiffness, and hardness of thin, hard films on soft substrates can be extracted. As demonstration of this, we show that FEM modeling can extract consistent values for the hardness and stiffness of a-tC films ranging in thickness from $400-10000 \AA$, whereas, the raw nanoindentation results give hardness and stiffness values that differ by over 100\%. This technique was developed in collaboration with industry leaders in the nanoindentation field, and is expected to become a standard analysis tool for improving the accuracy and reliability of nanoindentation results.

The third chapter of this report deals with advances made in cubic boron-nitride (cBN) synthesis. Prior to this project, the authors had developed a great deal of experience in the synthesis of $\mathrm{CBN}$ by a hybrid technique called ion-assisted pulsed laser deposition (IAPLD). Briefly this technique uses a pulsed laser to ablate a boron nitride target combined with a broad beam Kaufman-type ion source to supply nitrogen ions. The ion source is necessary to nucleate the cubic phase and sustain its growth. PLD alone will not nucleate and grow the cubic phase. Basic studies of the phase space for $\mathrm{CBN}$ nucleation and growth were carried out to map out the conditions under which this material could be grown. No mechanical properties had been optimized. The ion energies typically used for $\mathrm{cBN}$ growth to this point were from $500-1200$ $\mathrm{eV}$. Unfortunately, these energies produce too much damage in the growing film, and limit the crystalline quality of the films. Typically the films grew with nanocrystalline grains, and the grain growth appeared to be easily interrupted as the films increased in thickness. Early on in the project, it was found that the films grown with high ion energies were quite soft and not suitable for inclusion in multilayers. In addition, the residual compressive stress in the cBN films was also quite high and caused the films to delaminate at thicknesses near 0.1-0.2 $\mu \mathrm{m}$.

Two routes were followed to improve the mechanical properties of the films. The first was to develop a completely new deposition process and the second was to improve the IAPLD process. The new deposition process is called electron-cyclotron resonance (ECR) assisted sputter deposition. In this technique an argon RF plasma is used to sputter a $\mathrm{B}_{4} \mathrm{C}$ target as a source of boron. An ECR plasma is used to provide an independent source of nitrogen ions. The ions are accelerated to the sample by an independently controlled DC bias that is high enough to nucleate the cubic phase but not too high as to cause excessive ion damage. Thus, exact control of the deposition fluxes can be maintained to grow high quality films. In addition, the substrate temperature was raised to $1000^{\circ} \mathrm{C}$ to reduce residual stress in the films and increase the crystalline quality of the $\mathrm{cBN}$. This technique is naturally suited for making multilayers, as it is possible to grow $\mathrm{B}_{4} \mathrm{C}$ films by turning of the ECR source of nitrogen. Remarkably, the resultant films have a very low carbon content $(<2 \%)$ because carbon is not very miscible in $\mathrm{BN}$.

The cBN films produced by this technique were far superior to the old PLD process. The films were hard. Nanoindentation experiments combined with finite-element modeling showed 
that the films were slightly harder than bulk $\mathrm{cBN}$. The grain structure of the films was columnar, with much larger grain sizes, and no evidence of $h B N$ inclusions in the $c B N$ phase. The "gentler" growth process allowed for uninterrupted grain growth in the cBN phase which is crucial to improving the mechanical properties of the films. In addition, it was possible to grow much thicker films by this process. Films up to $0.8 \mu \mathrm{m}$ were grown that were adherent to the substrate. Unfortunately, the film nucleation still proceeded from the soft $\mathrm{hBN}$ phase, which still appears to be an intrinsic part of the growth process. Also, the growth rate of this technique was quite slow $(0.1 \mu \mathrm{m} / \mathrm{hr})$ limiting the practicality of using this process for growing multilayers.

Improvements in the IAPLD process were made in order to improve the quality of films grown by this process. For the first time, $\mathrm{x}$-ray reflectivity was used to characterize $\mathrm{cBN}$ films. This technique measures the thickness, density, and interfacial roughness in thin films and is well suited to analyze the intrinsically layered $\mathrm{cBN}$ system. In addition, in situ measurements of the stress buildup with growth were made to understand the effect of changing growth parameters on the final film stress state. The results of these studies were vast improvement in the quality of $\mathrm{cBN}$ films grown by the IAPLD technique. The improvements in the process produced films with crystalline quality similar to those produced by the ECR assisted sputter deposition technique but with the advantage of maintaining a higher growth rate.

The final two chapters of this report deal with theoretical work funded at the University of California at Berkeley. The work was to model dislocation flow in multilayer systems in order to promote a more fundamental understanding of hardness enhancements in multilayer systems. Our theoretical studies have focused on the role that sources play in the formation of pileups. Early on, it was determined that the problem of sources had not been treated adequately within the context of a grain size effect in a pure material. Since this is the logical starting point for further theory development, we began by including source information into the continuum theory of pileup formation. This problem, which we solved exactly, gives an expression for the strength of a material with a given grain size. To investigate the accuracy of the continuum approximation, we compare the results of the continuum theory with exact numerical results for a discrete dislocation model. The agreement between the continuum theory and the discrete dislocation model is excellent, implying that the continuum theory is a good description of pileup formation in systems with more than a few dislocations.

\subsection{Conclusions}

We have made tremendous advances in the quality of $\mathrm{cBN}$ and a-tC thin films. These advances have come about by applying the principles of basic research to the technical problem of growing hard multilayer thin films. We have applied new characterization methods to these systems (x-ray reflectivity, in situ stress characterization) that provide fundamental insight into the growth processes that have driven improvements in film quality. These advances have led to solutions to practical technological problems that we are actively pursuing with follow on research and development. In addition, we have successfully employed finite-element modeling techniques to solve a difficult problem of measuring the intrinsic hardness and stiffness of very hard films on soft substrates. This technique is already being employed by others and is well on its way to becoming a standard for analysis of nanoindentation results. Finally, we have improved the theoretical understanding of dislocation sources and pileup in materials. 


\section{Growth, characterization, and stress relaxation of amorphous tetrahedral carbon films}

\subsection{Introduction}

Understanding the nature of carbon films, and in particular those deposited by PLD is necessary to understand the results of this project. Therefore, this section explains the nature of the deposition process, evaluation of film properties using Raman spectroscopy, and the types of carbon films that can be deposited.

Our PLD process is capable of generating hydrogen free carbon films that range from amorphous glassy carbon (graphite-like -- soft and conductive) to diamond-like (hard and insulating). Control of the carbon bonding in the films is achieved by controlling the laser fluence on the target. For high laser fluences, the ablated carbon species from the graphite target have high energies $(-100 \mathrm{eV})$. The diamond-like properties result from the subsurface penetration of these energetic species resulting briefly $(\sim 1-5$ picosecond) in local regions of very high pressure and temperature - termed a "thermal spike". Under these conditions, diamond-like four-fold coordinated carbon bonds are favored over graphitic three-fold coordinated bonds and a complex amorphous diamond-like structure is built up. This is a highly metastable film growth process and the atoms in the films can and do end up in highly non-equilibrium positions. The resultant films are quite smooth (surface roughness $<1 \mathrm{~nm}$ ) and hard (90 Gpa) with resistivities approaching $10^{7} \Omega \mathrm{cm}$. Unfortunately, these films typically have high degrees of residual stress $(>5 \mathrm{GPa})$.

For low laser fluence, the carbon species are much less energetic, and they do not penetrate the subsurface region. This results in a more conventional surface film growth where the atoms are allowed to find positions closer to equilibrium values. The resultant films are rougher, soft, less resistive $(<10 \Omega \mathrm{cm})$, and lower stress $(1-2 \mathrm{GPa})$.

Stress control is a key issue with a-tC films. The high degree of residual stress is incompatible with many of the applications projected for this material. For example, it is impossible to grow these films to $>0.2 \mu \mathrm{m}$ on Si substrates due to adhesion failure because of the high stress and also impossible to make free standing membranes of this material. Importantly, high stress severely limits the ability to grow multilayer structures, again due to adhesion problems.

One common technique for relieving stress is to anneal films at temperatures higher than the growth temperature (in this case room temperature). We have annealed in situ high quality amorphous-tetrahedrally-bonded carbon (a-tC) films with high residual compressive stresses $(\sim 7$ $\mathrm{GPa}$ ) for varying times $(12-70 \mathrm{hrs})$ and temperatures $\left(100-600^{\circ} \mathrm{C}\right)$ and measured the stress relaxation. We find that annealing samples to $600{ }^{\circ} \mathrm{C}$ for short times (2-5 minutes) relieves $\sim 95 \%$ of the residual compressive stress. As the annealing time increases, the stress reverses sign and the film goes into tension. The stress changes are irreversible upon cooling indicating they are not due to thermal mismatch (a relatively small thermal mismatch does exist) with the underlying silicon substrate. Surprisingly, Raman, EELS, and electrical resistivity measurements performed on annealed films show little change compared to the as-grown samples (subtle changes are present) indicating the a-tC samples have retained their diamond-like properties.

Previously, we derived a model for the stress relaxation as a function of time and temperature. 1 The model is based upon first order kinetics and assumes that there is a distribution of activation energies for the stress relaxation and that the relaxation is driven by a reduction in the strain energy associated with the conversion of a 4- to 3-fold carbon atom. We show that this model can be used to fit the time and temperature stress relaxation data over the entire range studied. From this model, we have predicted that $~ 7 \%$ of the 4 -fold atoms must convert to 3-fold for complete stress relaxation. Also, we have derived the energy barrier distribution for relaxation and find that it extends from $\sim 0.7-3.5 \mathrm{eV}$ peaked near $1.8 \mathrm{eV}$.

With the ability to stress relieve the samples, one can then grow thick samples by 
repeated room temperature film growth followed by thermal annealing to relieve UVExcimer residual stress. Using this technique, $w \in{ }_{i-120}^{2} \mathrm{~J} / \mathrm{cm}$

have grown up to $10 \mu \mathrm{m}$ thick a-tC films on Si(100) substrates. The resultant films are adherent to the substrate with low stress (< $0.2 \mathrm{GPa}$ tensile). Raman spectra from thick specimens show little change from the thin as-grown films. Using a nanoindenter combined with finite element modeling, we find the thick a-tC films to be very hard $(\sim 88 \mathrm{GPa})$ and stiff (elastic modulus E 1100 $\mathrm{GPa})$.

\subsection{EXPERIMENTAL}

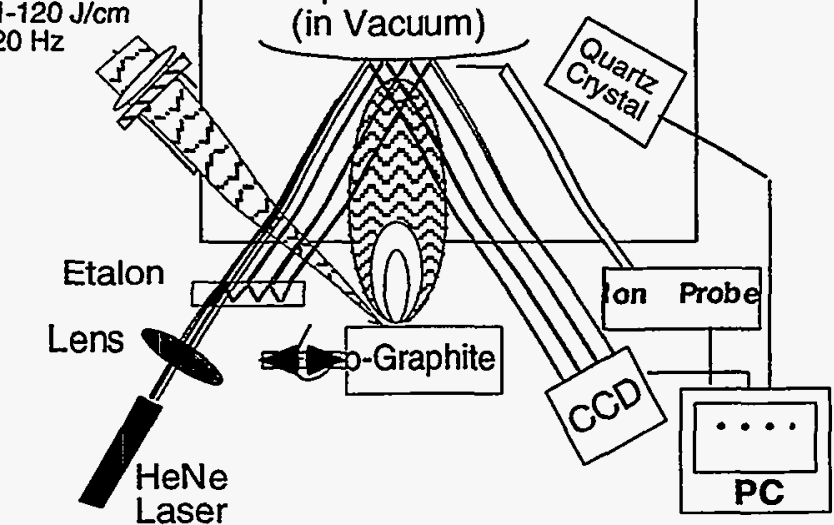

The PLD vacuum chamber (see Fig Fig. 2-1 Schematic of the deposition apparatus, with $2-1)$ is capable of attaining a base pressure

of $1 \times 10^{-6} \mathrm{~Pa}$. The pyrolytic graphite targets (Advanced Materials) were configured in a cylindrical geometry, with the laser striking the axis of the cylinder, and the targets simultaneously rotated and translated to prevent cratering and maintain uniform deposition conditions from run to run. The laser was a two-cavity-excimer ( $\mathrm{KrF}$ gas fill lasing at $248 \mathrm{~nm}$ with oscillator pumping amplifier in the "power" configuration) capable of generating 600-mJ pulses of 17-ns duration with a low divergence $(<0.2-\mathrm{mrad})$ and high beam quality. The laser light was focused into the vacuum chamber using a spherical lens with a $35-\mathrm{cm}$ focal length. The laser beam illuminates the rotating target at a $45^{\circ}$ angle from the target normal. By varying the total energy in the laser pulse at a fixed spot size (just past the actual focal distance of the lens), the laser fluence on the target was varied from 5 to $125 \mathrm{~J} / \mathrm{cm}^{2}$. The laser repetition rate was $20 \mathrm{~Hz}$ and the target to substrate distance was $11 \mathrm{~cm}$. At the highest fluences, the growth rate over a $2.5 \mathrm{~cm}$ diameter circular substrate was $\sim 1.0 \AA / \mathrm{sec}$ implying that each laser pulse yielded $\sim 0.05 \AA / \mathrm{sec}$, a submonolayer coating. Before deposition, the uncoated $\mathrm{Si}(100)$ substrates ( 2.5 or $5.0 \mathrm{~cm}$ diameter p-doped with $B$ to $0.02 \Omega \mathrm{cm}$ ) were cleaned to remove the surface oxide layer by a wet dip procedure in a $\mathrm{HF} / \mathrm{NH}_{4} \mathrm{~F}$ solution ${ }^{2}$.

In addition, a limited number of samples were grown using a filtered-arc deposition apparatus (Commonwealth Scientific). The films were grown with an arc current of $100 \mathrm{Amps}$, and $\mathrm{DC}$ bias on the deposition substrate ranging from $0-150 \mathrm{~V}$.

Ions in the laser plume were characterized by using a fast ion probe (response time $<5$ $n s)$.. The probe was simply a copper wire that was negatively biased $(-30 \mathrm{~V})$ with respect to ground in order to repel electrons. The output from the probe was fed into a digitizing oscilloscope at a resolution of $20 \mathrm{~ns}$. The oscilloscope was triggered by a fast Si photo-diode (response time $<5 \mathrm{~ns}$ ) that was placed to intercept some of the stray laser light. The ion probe was placed in the position of the sample $11 \mathrm{~cm}$ from the target. Thus the arriving ion flux as a function of time and fluence on the target was recorded.

Raman spectroscopy and electron energy loss spectroscopy (EELS) were used to characterize the atomic bonding of films both after growth and after annealing. Raman spectroscopy is particularly useful in that it is a nondestructive technique and relatively routine to employ. For films grown in this study we used 458 or $514 \mathrm{~nm}$ light at $50 \mathrm{~mW}$ of power. Fig. $2-2$ is a compilation of standard Raman spectra for various forms of carbon from graphite to diamond. For graphite there are two active modes at $1350 \mathrm{~cm}^{-1}$ and $1581 \mathrm{~cm}^{-1}$. The Raman band at $1580-1590 \mathrm{~cm}^{-1}$ (the "graphite" or " $\mathrm{G}$ " peak) is a fundamental Brilloun-zone-center mode of graphite. The Raman band at $1350-1360 \mathrm{~cm}^{-1}$ (the "disorder" or "D" peak) is believed to be a Brilloun-zone-edge phonon mode that intensifies for graphite crystal domains less than $100 \mathrm{~nm}$ in size. Glassy carbon is a disordered graphitic phase with broad Raman peaks near 
$1360 \mathrm{~cm}^{-1}$ ("D") and $1590 \mathrm{~cm}^{-1}$ ("G"). Diamond has one active Raman mode at $1332 \mathrm{~cm}^{1}$ characteristic of sp3-bonded carbon. Conventional DLC with hydrogen has a broad asymmetric Raman peak near $1515 \mathrm{~cm}^{-1}$ with a long tail that extends down to $-1000 \mathrm{~cm}^{-1}$. a-tC also has a single broad Raman mode, but this mode is shifted up to higher wavenumber $\left(-1565 \mathrm{~cm}^{-1}\right)$ and is more symmetric than that of a-C:H. This peak arises from $\mathrm{sp}^{2}$-bonded carbon atoms. For diamond-like carbon, the presence of $\mathrm{sp}^{3}$-bonded carbon is not directly detected by Raman spectroscopy using visible light as the excitation source due to resonance enhancement of the $\mathrm{sp}^{2}$-bonded atoms at the laser wavelength used. This results in a 50x increase in the intensity of the graphitic component of the films over the diamond-like. The four-fold coordinated carbon signal is obscured by strongly resonance-enhanced signals from the $\mathrm{sp}^{2}$-like bonds ${ }^{3}$. (The picture changes if one uses ultra-violet (UV) light. The more energetic photons are less strongly absorbed by the 3-fold bonded material and it is possible to detect 4-fold bonded carbon.)

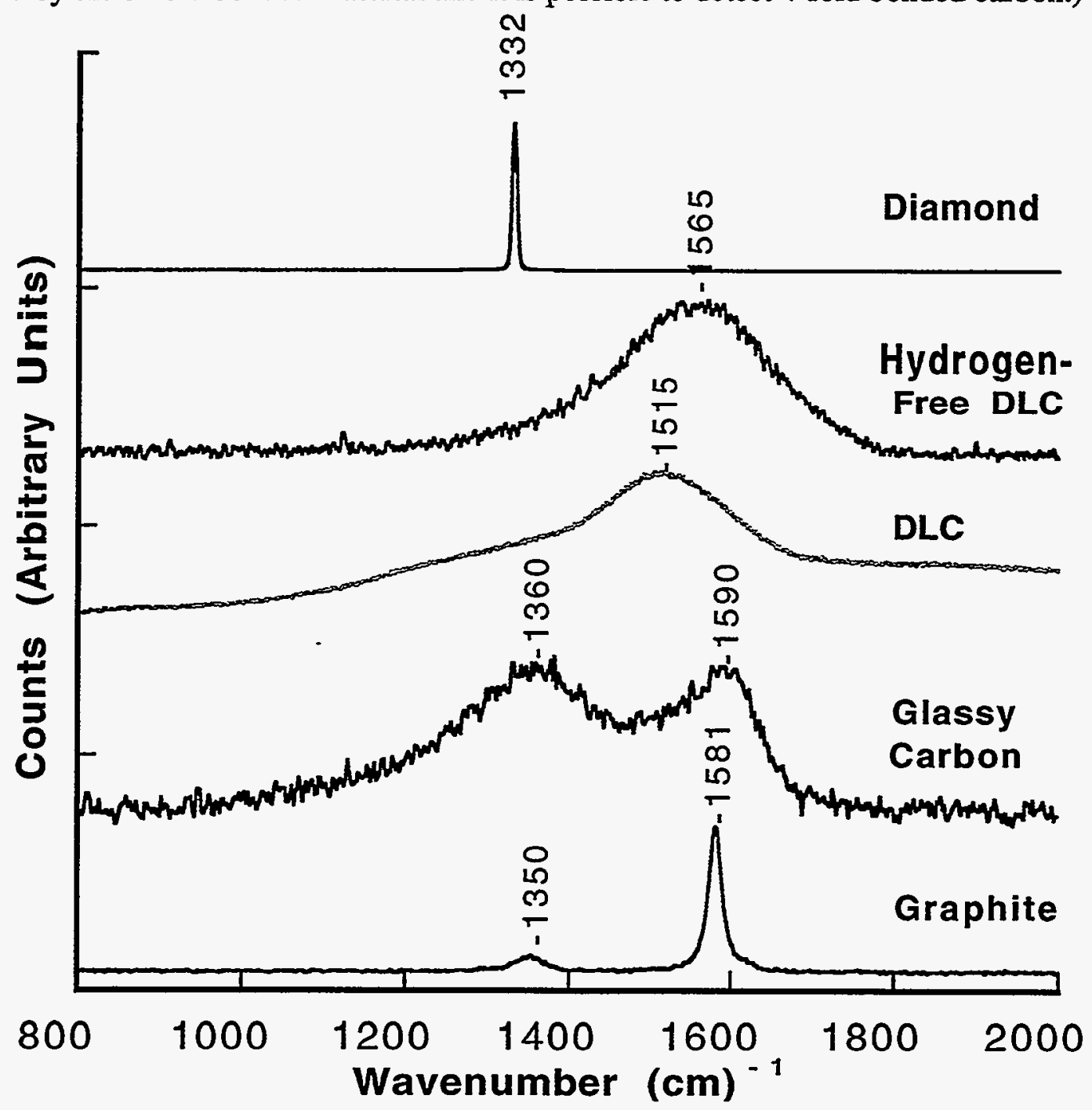

Fig. 2-2 Representative Raman spectra from various forms of carbon

EELS requires some sample preparation and is not quite routine, yet provides a useful look at both the 3- and 4-fold hybridization. The plan-view EELS samples (a-tC thickness $1100 \AA$ ) were prepared by chemically etching away the silicon substrate with a 5:3:3 solution of $\mathrm{HNO}_{3}, \mathrm{CH}_{3} \mathrm{COOH}$, and $\mathrm{HF}$. Plasmon and carbon K-edge spectra were acquired on a series of specimens annealed for 30 minutes in argon to varying temperatures up to $600{ }^{\circ} \mathrm{C}$. The resolution of the EELS instrument was $\sim 1.3 \mathrm{eV}$. 
X-ray reflectivity has been used extensively to analyze the structure of layered thin films as has been described in detail elsewhere. ${ }^{46}$ Briefly, the intensity of specularly reflected $\mathrm{x}$-rays depends upon the electron density at and near the film surface. ${ }^{7}$ Above the critical angle for total external reflection, the $\mathrm{x}$-rays penetrate and interfere constructively or destructively with reflections from buried interfaces. The observed interference fringes can be simulated using Fresnel recursive relations modified to include interface roughness. ${ }^{4,8}$. A non-linear least squares fit of the calculated intensity to the measured intensity determines the electron density, thickness $t$, and roughness $\sigma$ of each layer. ${ }^{4,8}$ The mass density $\rho$ is then deduced using the stoichiometry of the individual layers. ${ }^{4}$ In the case of these carbon films, the only constituent atom is carbon which greatly simplifies the density measurements. Our measurements were performed on a four-circle Philips X-Pert MRD diffractometer using $\mathrm{Cu} \mathrm{K} \alpha$ radiation from a fixed anode line source. A parallel plate collimator, $100 \mu \mathrm{m}$ slit and flat graphite monochrometer defined the reflected beam. The sample height was adjusted to bisect the beam using a stage capable of 0.1 micron steps. A $\theta-2 \theta$ scan was performed to measure the specularly reflected intensity.

Stress determination was made by two techniques. Ex situ measurements of film thickness and wafer curvature using a stylus profilometer and Stoney's ${ }^{9}$ equation. In situ measurements of the residual stress were made using a novel laser curvature technique. ${ }^{10}$ Fig. 21 shows a schematic of the technique. A HeNe laser beam is split into parallel beamlets by an etalon. The beamlets reflect off the center of the sample and the centroid position of each is recorded by a CCD read by a computer. The advantage of the parallel beamlets are two-fold. First, no scanning of the laser beam is required; and second there is much reduced sensitivity to vibrations since coherent vibrations of the spots due to most vibrations are essentially eliminated as a source of noise. The technique can measure changes in radius of curvature at a sensitivity of $\sim 5 \mathrm{~km}$. In addition, simultaneous measurements are recorded of the sample thickness from a quartz crystal monitor and temperature from an optical pyrometer. Thus, we can measure the stress build-up with growth, and stress reduction with annealing time and temperature.

\subsection{DISCUSSION}

\subsubsection{Characterization}

\subsubsection{1 lon probe}

Fig. 2-3 depicts a plot of the ion probe data for increasing laser fluence. The raw probe output is measured current versus time from the laser trigger pulse. The data is converted into current density versus ion energy using simple geometric considerations (target to probe distance, probe area) and assuming that the ions are comprised solely of singly ionized carbon atoms. The number of ions, peak ion energy, and ion energy distribution all increase with increasing fluence. As will be seen below, the highest fluences correlate with the most diamondlike films and thus, also with higher average energy of the ablated species. The data show that the carbon ion energies are broadly distributed over a wide range at all fluences. 
At the highest fluences, one can estimate from the measured ion current density and the final thickness and film density that roughly $10 \%$ of the atoms incorporated into the film are ions. The ion probe does not give any information as to the makeup of the other $90 \%$ of the ablation plume. It is reasonable to surmise that as the laser fluence increases the average energy of the neutral species in the plume will increase in a similar fashion to the ions but the makeup of these species (e.g. radicals, neutral atoms, clusters, etc.) has not been determined. Murray et al. ${ }^{11}$ used time-of-flight mass spectroscopy to look at neutral species in ablated carbon plumes and found that the number of large carbon clusters decreased with decreasing laser wavelength, and increasing laser fluence. Since the fluence used here is much higher than that reported in Murray et al., the neutral species in the plume should be quite energetic and comprised of single atoms with a few $\mathrm{C}_{3}$ clusters.

It is interesting to compare the ion probe data of Fig. 2-3 with results from other growth techniques used to grow a-tC films. The mass selected ion beam (MSIB) deposition results by Lifshitz ${ }^{12}$ and Hofsäss ${ }^{13}$ show that the most diamond-like films are grown near ion energies around $100 \mathrm{eV}$. MSIB offers the most precise control of growth energetics, where ion energy (and even isotope) can be precisely controlled. There is virtually no contribution from neutral carbon deposition. The trade-off is a low overall growth rate over small substrate areas. Importantly, the MSIB results show a broad maximum from $\sim 50-300 \mathrm{eV}$ where reasonably good-quality a-tC films can be grown. Interestingly, this ion range correlates well with the ion range measured by the ion probe in the PLD process. Another popular growth technique is called filtered-arc deposition. ${ }^{14-17}$ In this technique, an arc discharge is used to ablate carbon material off of a graphite cathode. The ablated species contain a high fraction of singly ionized carbon atoms that form a plasma. The plasma is then filtered using magnetic fields to bend the ions around a curved duct causing the neutral species to strike the walls and thus removing them. What emerges is a nearly $100 \%$ ionized carbon plasma with a potential around $20 \mathrm{~V}$. Substrate biasing is then used to increase the ion energy. Typically the best a-tC films are found around $100 \mathrm{eV}$ with a narrower range of ion energy $(50-150 \mathrm{eV})$ for the Fig. 2-3 Current density versus ion energy as a function of production of good quality a- increasing laser fluence $(5-125 \mathrm{~J} / \mathrm{cm})$. Both the average energy tC films. The carbon ion and peak width increase with fluence. Note the best a-tC films are energy is not as well defined produced at the highest fluences.

as in the MSIB case, but

much more narrowly defined than in the PLD case. The expected energy spread is probably not more than $10 \mathrm{eV}$. One great advantage of the filtered-arc technique is the high deposition rates (1-3 amps deposition current is readily achievable) and the ability to raster the plasma over large areas by using magnetic fields. The disadvantages are that high currents can cause heating, and the filtering is not always easy to control, nor does it always remove all the particulates. The main difference between these two techniques and PLD is that nearly all the film is grown with ions and the ion energy is well defined. For the PLD case, $90 \%$ of the deposition flux is not ionized, and the ion energy spread can be quite broad. 


\subsubsection{Residual Growth Stress}

Fig. 2-4 depicts a plor of residual compressive stress (determined by stylus profilometry) as a function of the laser fluence on the target. The stress starts out low at low fluence $(1 \mathrm{GPa})$ and rises to nearly $8 \mathrm{GPa}$ at the highest fluences. The large compressive stresses generated are typical of a-tC materials and are comparable (although slightly lower) to those reported by researchers using other growth techniques ${ }^{12,18}$ where the highest residual stresses are usually in the 10$12 \mathrm{GPa}$ range. \{These lower stresses may be intrinsic to the

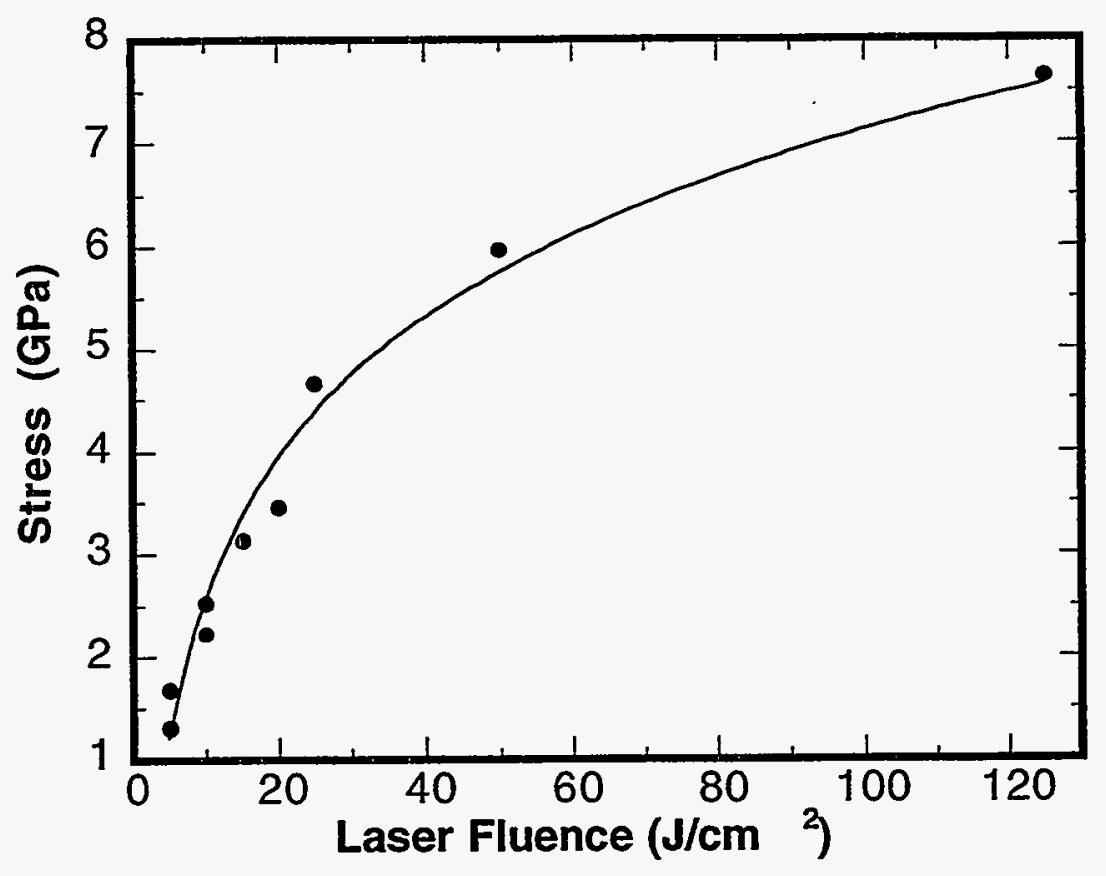

PLD process because the Fig. 2-4 Residual compressive stress as a function of laser measured ion energy spread is fluence. The line is a guide to the eye.

very large. This large spread

means that not all ions are at the "optimum" energy, thus lowering the overall stress. This also implies that PLD grown films may have lower 4-fold content, which is confirmed by EELS measurements (see below).\} Even so, the residual stresses are large and they typically cause the films to delaminate from Si substrates at thicknesses approaching $2000 \AA$ due to adhesion failure at the film substrate interface. These stresses are a real problem with a-tC films as they limit the ability to grow thick films, multilayers, and produce free-standing membranes of this material. To date, no reports have been made of as-grown stress-free a-tC material. It appears that stress is intrinsic to the growth process and cannot be avoided. The stress is due to the high ion energy of the bombarding carbon species. At energies above $20 \mathrm{eV}$ the carbon ions will start to penetrate the surface in a process commonly called "subplantation."12,19 Several models have been proposed for the mechanism of 4-fold bonding creation based upon this sub surface penetration. The models differ on whether the stress is the intrinsic driver of the transformation of 3 -fold to 4-fold, or is simply a byproduct of the process, and whether dynamic considerations such as "thermal spikes" play a significant role. For a review see Mirkarimi et al. ${ }^{20}$

\subsubsection{Raman Spectra}

Three representative Raman spectra from these films are shown in Fig. 2-5. These spectra were obtained with visible $(514 \mathrm{~nm})$ laser light excitation. As the laser fluence increases, the Raman spectrum of atC evolves from a very broad asymmetric band near $1550 \mathrm{~cm}^{-1}$, to a narrower more Fig. 2-5 Raman spectra for three samples grown at symmetric band centered near $1575 \mathrm{~cm}^{-1}$. different laser fluence.

The upward peak shift is due in part to the

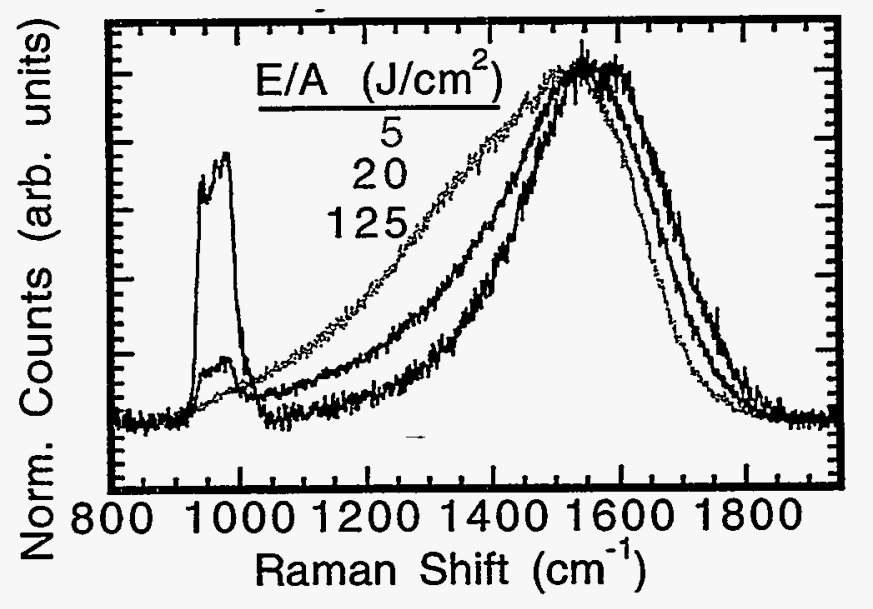


increased stress in the films, ${ }^{21}$ but the change in peak shape indicates that the character of the three-fold bonded carbon is changing as the films become more diamond-like. The second order Si band at $965 \mathrm{~cm}^{-1}$ also increases dramatically in intensity with increasing laser fluence. Since these three films were all $\sim 1000 \AA$ in thickness, the increase in intensity is due to an increase in the transparency of the a-tC films with fluence. Doyle and Dennison ${ }^{22}$ have calculated the vibrational frequencies of various isolated rings of carbon atoms with varying numbers of carbon atoms. They then used these frequencies to fit the Raman spectra of various carbon based films. Similar efforts to fit the visible Raman spectra of a-tC films have been made. ${ }^{23}$ The results suggest that the change in the Raman spectra with increasing fluence is a change from a structure with 5-, 6- and 7-member carbon rings to one that shows predominantly 6-membered rings. As the structure becomes more dominated by 4-fold carbon, the 3-fold bonding becomes more specific, as if limited to certain bonding conditions by the presence of the 4-fold matrix. Raman measurements with ultra-violet excitation ${ }^{24}$ (more sensitive to 4-fold bonding) also indicate a similar picture. The 4-fold content increases with fluence, and the 3-fold bonding becomes more specific as the number of 3-fold sights diminish.

\subsubsection{EELS}

Fig. 2-6 depicts the k-edge EELS spectra taken from two samples grown at low and high fluence. The intensity of the $\pi^{*}$ peak relative to the $\sigma^{*}$ peak is an indication of the relative abundance of 3- to 4-fold bonding in the films. The relative intensity of the $\pi^{*}$ peak decreases dramatically with laser fluence again consistent with an increase in the 4-fold coordinated carbon. The $\sigma^{*} / \pi^{*}$ peak height ratio as a function of fluence is plotted on the left axis of Fig. 27. Initially, there is a large increase in the 4-fold bonding with fluence, followed by a much slower increase at the higher fluences. Fallon et al. ${ }^{17}$ describe an empirical technique for calculating the actual 4-fold bonding content from the measured k-edge EELS spectra. Using their technique, the high fluence sample has a 4 -fold content of $\sim 75 \%$. This compares to a measured 4 -fold content of $\sim 85 \%$ for the filtered arc a-tC samples measured in their study. ${ }^{17}$ The lower 4-fold content may be intrinsic to the PLD process since the energy spread of the ions is so broad that a large fraction of them are off of the optimum ion energy, thus making it difficult to achieve higher 4-fold content. On the other hand, it also should be noted that perhaps with a higher fluence or shorter wavelength it may be possible to produce samples with a higher 4-fold content.

Low energy loss spectra were also recorded to measure the position of the plasmon peak energy. The plasmon energy is essentially a measure of the density of conduction electrons in the sample, and for a single element carbon film, can be directly converted into mass density. The results of the density measurement versus fluence are recorded on the right axis in Fig. 2-7.

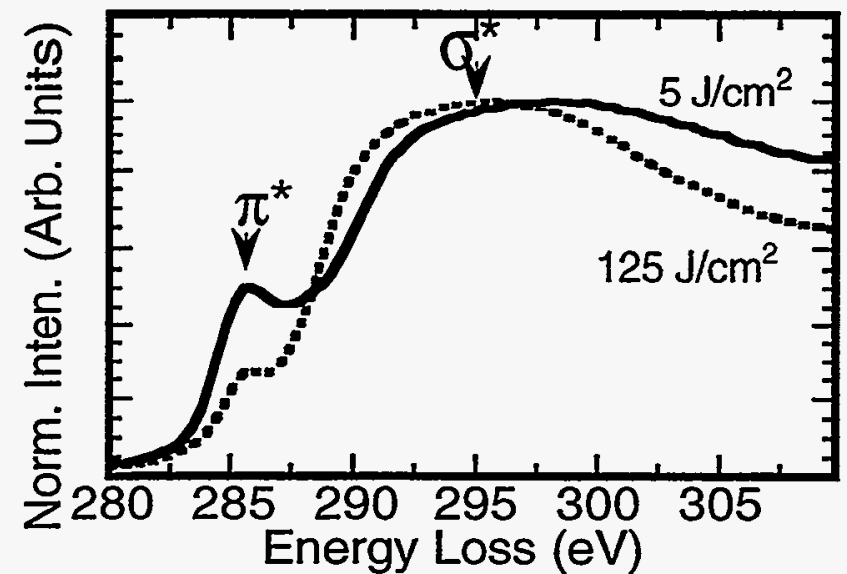

Fig. 2-6 a.) K-edge EELS spectra for two samples grown at low and high laser fluence. 
The mass density rises from $\sim 2.2$ $\mathrm{g} / \mathrm{cm}^{3}$ at the low fluence to near 3.2 $\mathrm{g} / \mathrm{cm}^{3}$ at the high fluence. For reference, the density of graphite is $2.2 \mathrm{~g} / \mathrm{cm}^{3}$ and the density of diamond is $3.5 \mathrm{~g} / \mathrm{cm}^{3}$. The density increase mirrors almost exactly the increase in the $\sigma^{*} / \pi^{*}$ ratio with fluence and indicates that density measurements should provide a reasonable estimate of the ratio of 4to 3-fold bonding in the samples.

\subsubsection{X-ray reflectivity}

$X-r a y$ reflectivity

measurements of the sample density were performed on the same set of samples used for the EELS and Raman measurements. The X-ray reflectivity spectra were fit to Fig. 2-7 Comparison of $\sigma^{*} / \pi^{*}$ ratio, plasmon density, and determine the sample mass density, $x$-ray reflectivity density as a function of laser fluence. and the results are plotted on the left

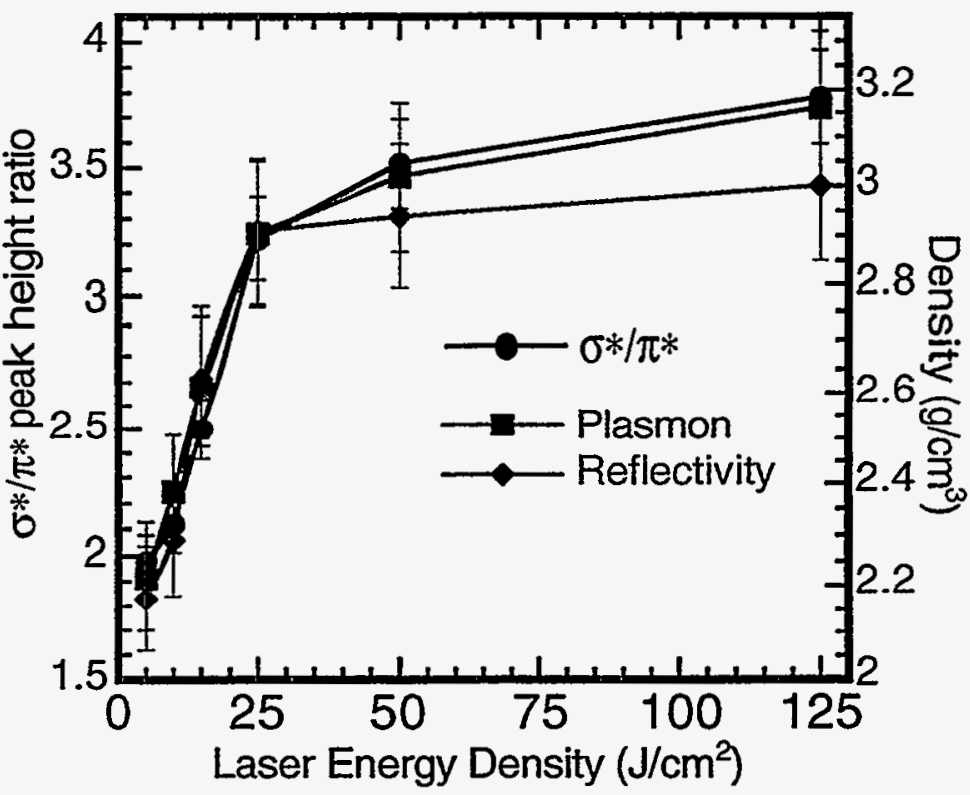
axis of Fig. 2-7. The density determined at low fluence agrees quite well with the density as determined from the plasmon peak position. At the higher fluences, the two curves diverge, but are still within $\sim 5 \%$ of each other. The good correlation between the two techniques is not surprising, since both are looking at electron densities. For the plasmon measurement, one is looking at conduction electrons, and for the x-ray reflectivity the entire electron cloud is probed. The mass density determined from these two approaches should be the same. Importantly, the demonstration of this correlation allows the 4- to 3-fold bond ratio to be inferred from a x-ray reflectivity measurement of the density. This is advantageous since the reflectivity measurement is nondestructive and fast compared to the slightly more complex EELS measurement.

Also, on the right axis of Fig. 2-8 is a plot of the measured sample density determined by $\mathrm{x}$-ray reflectivity versus the growth stress. At low density, the stress is relatively low, and large increases in density result in relatively minor increases in stress. Near $-2.8 \mathrm{~g} / \mathrm{cm} 3$ and $4 \mathrm{GPa}$ stress, the curve bends over such that smaller increases in the density result in large increases in stress. From a local perspective, at low density the carbon network is relatively free to rearrange

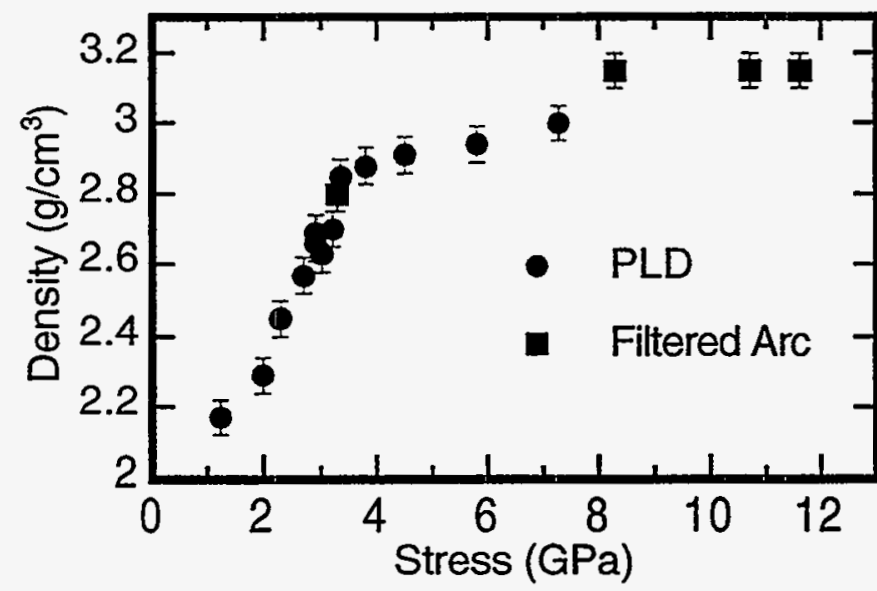

Fig. 2-8 X-ray reflectivity density vs. stress for samples grown by PLD and filtered arc. itself to relieve the stress induced by the implanting carbon atoms. It can do this by atomic bond rearrangements that result in a structure with less volume per atom thus minimizing the strain in the network. Several possibilities exist. For example, the 3-fold carbon atoms can form shorter bonds to each other (e.g. carbon double bonds), 3-fold atoms can convert to 4-fold, and/or clustering may occur. In support of these observations, the UV Raman measurements ${ }^{24}$ show that as the density increases, the carbon atoms tend to form more 4-fold atoms, and that the 3-fold atoms form into more isolated 3-fold structures that have higher vibrational energy. As long as the network is 
underconstrained and there are 3fold sites available for conversion, the density can increase rapidly with relatively small stress increases. When these mechanisms are used up, the network becomes overconstrained and the stress builds more rapidly with density. Clearly, dynamics play a role in this problem as atoms must be allowed to migrate to the surface (possibly through a stress driven process) otherwise the density would build up without limit.

Overall, the ion probe, stress, Raman, EELS and X-ray reflectivity measurements indicate that we can tailor the bonding in the amorphous carbon films. By changing the laser energy density, we can make films that range in density from $2.2-3.2$ $\mathrm{g} / \mathrm{cm}^{3}$, and have varying degrees of diamond-like character. The stress, density, and bonding are all correlated to the energetics of the ablation plume.

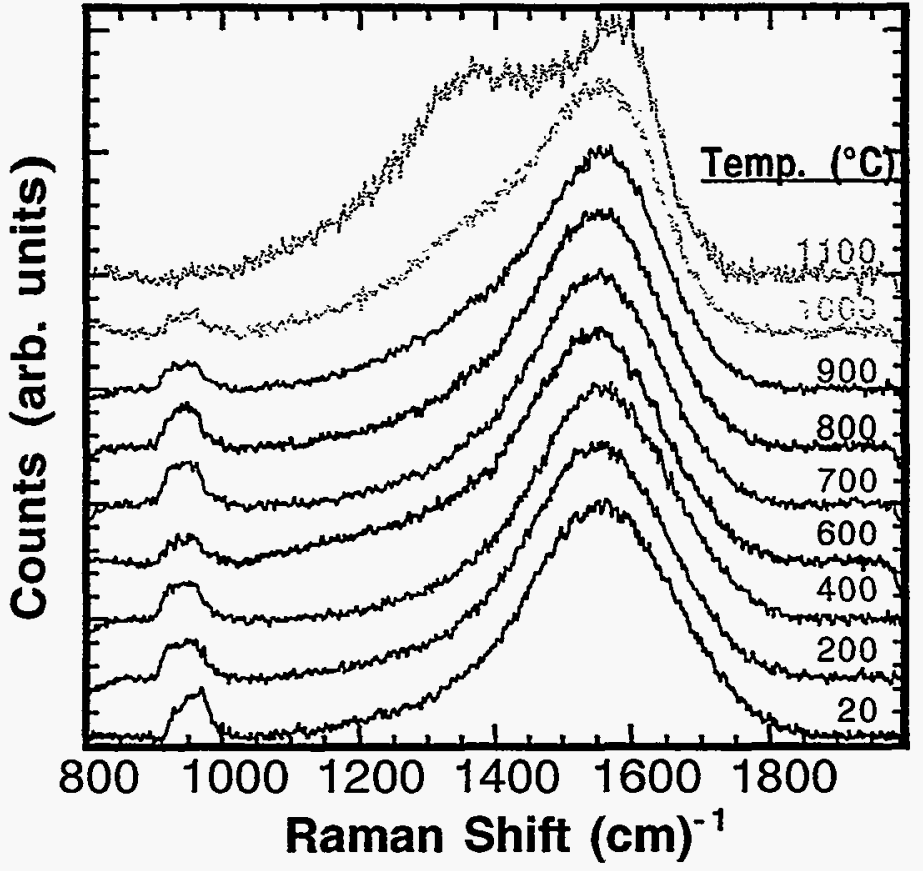

Fig. 2-9 Raman spectra of a a-tC film heated in vacuum to increasing temperature. The films were heated for 30 minutes at each temperature then cooled to room temperature for measurement of the Raman spectra.

\subsubsection{Thermal Annealing of a-tC films grown at high fluence}

We studied the changes in film properties with thermal annealing in vacuum and inert environments with Raman, EELS and stress measurements. The results show that a-tC films are remarkably stable up to annealing temperatures approaching $800^{\circ} \mathrm{C}$. In particular, only subtle changes are found in the bonding.

Remarkably, we find that the residual stress in these films can be completely relieved.

The thermal stability of these films was studied by Raman spectroscopy and is reported elsewhere. $^{25}$ Briefly, fig. 2-9 depicts a plot of the Raman spectra of an a-tC film grown at high laser fluence subjected to thirty minute anneals at increasing temperature intervals. Significantly, there are no major changes in the a-tC Raman peak

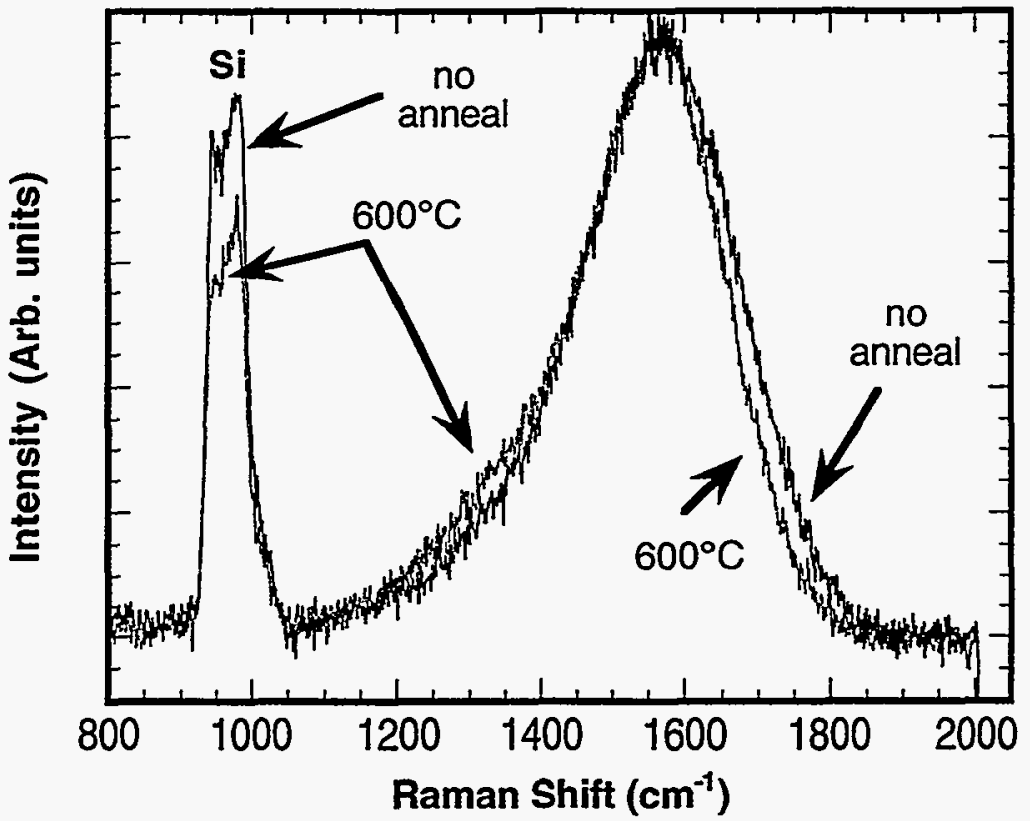

position and shape till $800^{\circ} \mathrm{C}$. Fig. 2-10 Raman spectrum of as-grown and annealed singleHowever, the second order Si layer a-tC films. 

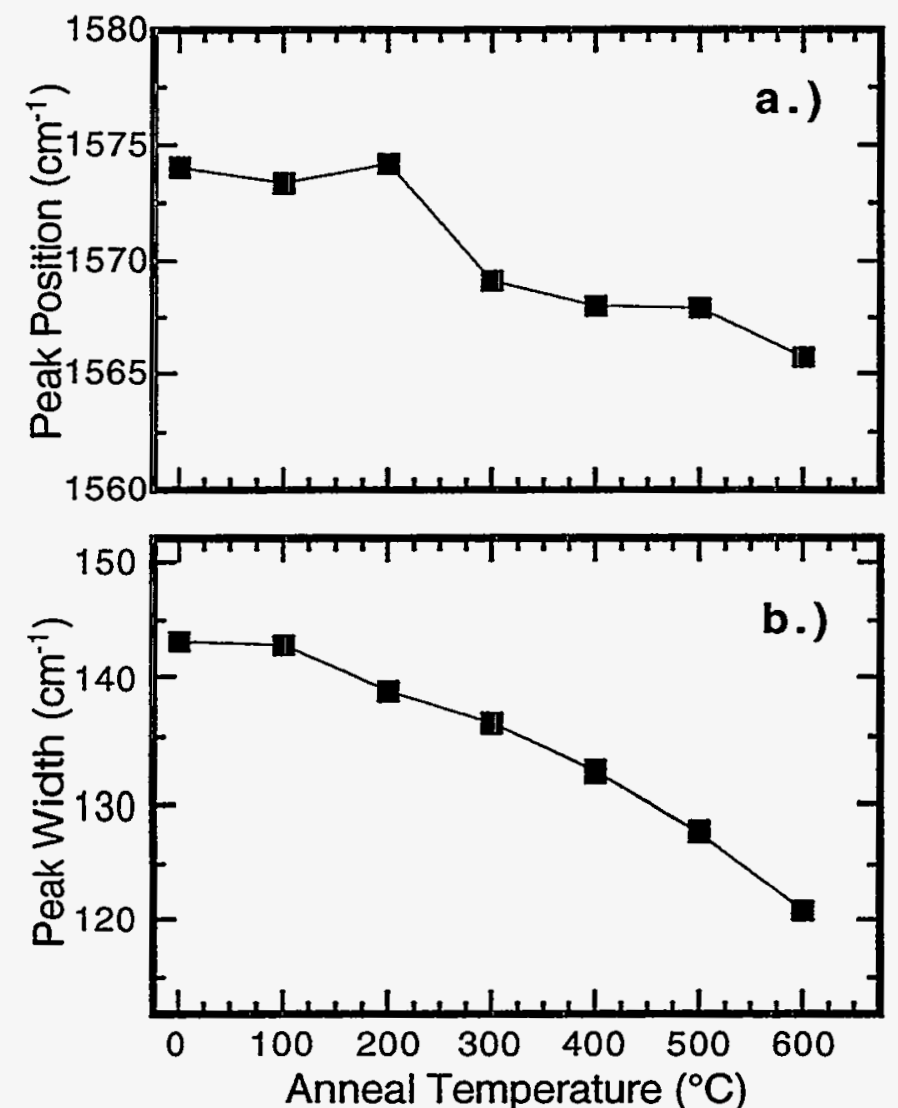

Fig. 2-11 a.) Raman peak position as a function of anneal temperature. b.) Raman peak width as a function of anneal temperature. band at $980 \mathrm{~cm}^{-1}$ shows a decrease in intensity that is detectable at the lowest temperatures studied. This decrease in intensity is associated with an increase in absorption in the film and thus an increase in the 3-fold bonding in the films. The magnitude of the absorption is highly sensitive to the 3-fold bonding and small changes can have a great affect. Above $800^{\circ} \mathrm{C}$, the sample shows major bonding changes and the spectra evolve to that of nanocrystalline graphite.

A more direct comparison of the Raman spectra from as-grown and annealed samples are shown in Fig. 2-10. Since the two samples were cleaved from the same wafer, the changes in the Raman spectra are associated with changes induced by the annealing and not variabilities in the sample preparation. The main Raman active mode $\left(1574 \mathrm{~cm}^{-1}\right)$ actually narrows asymmetrically $\left(23 \mathrm{~cm}^{-1}\right)$ and shifts downward $\left(8 \mathrm{~cm}^{-1}\right)$ after annealing. (The shifts were determined by fitting a single gaussian to the data between 1150 and $1800 \mathrm{~cm}^{-1}$.) In addition, there is an increase in intensity near $1350 \mathrm{~cm}^{-1}$ and an increase in the opacity of the samples as determined by the decrease in intensity of the 2 nd order $\mathrm{Si}$ Raman band. A plot of the Raman band

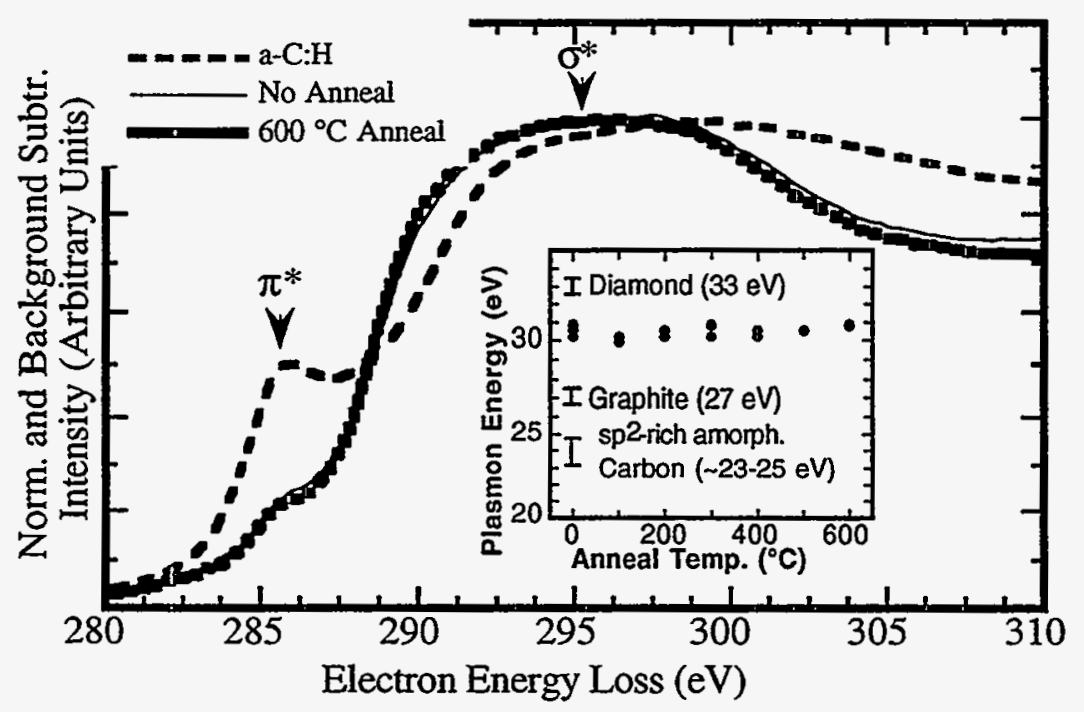

Fig. 2-12 Carbon K-edge EELS spectra for a-tC samples The inset shows the plasmon energy vs. anneal temperature as determined from the low energy loss spectra. peak width and shift with annealing temperature is shown in Fig. 2-11.

At least two factors affect the Raman spectra. The first is the simple relief of stress. In principle, this should cause the peak of the Raman band to shift to lower wavenumber, as the compressive stress decreases. Also, bonding changes in the film will affect the shape and position of the bands and an increase in the 3-fold content will decrease the intensity of the 2 nd order Si band. We note that the end result of the annealing is the full conversion of a sample with predominantly 4-fold bonding to a nanocrystalline graphite phase 
(Fig. 2-9) at annealing temperatures above $800^{\circ} \mathrm{C}$. The main peak position of nanocrystalline graphite is $1581 \mathrm{~cm}^{-1}$, thus one would expect an increase in the peak position with annealing as well as an increase in the intensity of the disorder band near $1380 \mathrm{~cm}^{-1}$. This peak shift is opposite of that expected for stress relief without significant bonding changes.

Stress relief due to spallation (at room temperature) has been shown to change the a-tC Raman band. ${ }^{21}$ In contrast to annealed samples, the Raman band shift $\left(20 \mathrm{~cm}^{-1}\right)$ is symmetric with no change in the peak width. Experimentally, the band shift is determined to be $\sim 1.9$ $\mathrm{cm}^{-1 / G P a}$ of stress relieved ${ }^{21}$. For our annealed samples, the peak shift is $8 \mathrm{~cm}^{-1}$ indicating a stress relief of $4.2 \mathrm{GPa}$. This is slightly more than half of the $7 \mathrm{GPa}$ of stress relieved in the sample. Bonding changes in the film due to the annealing would cause the peak position to shift upward. The spallation allows the carbon atoms in strained configurations to relax nearer to equilibrium conditions, as opposed to the thermal annealing which results in a combination of stress relaxation and C-C hybridization changes.

Fig. 2-12 shows the carbon K-edge transmission EELS spectra for the as-grown and annealed samples along with an a-C:H sample grown by a plasma chemical vapor deposition process. Due to multiple inelastic scattering (the a-tC films were 1.5 times the electron mean free path thickness) absolute quantification of the $\mathrm{K}$-edge spectra is inconclusive. However, the $\pi^{*}$ peak height relative to the $\sigma^{*}$ was unchanged between samples indicating only minor changes with annealing.

EELS spectra of the plasmon region were also recorded (inset fig. 2-12). The plasmon energies (between 30 and $31 \mathrm{eV}$ ) remained constant for the as-grown and annealed samples. EELS work by Fallon et $\mathrm{al}^{17}$, indicates that this corresponds to $\sim 75 \% \mathrm{sp}^{3}$-bonding. For contrast, the low-density sp2-rich a:C-H film plasmon peak energy was $23 \mathrm{eV}$.

We have also measured the resistivity and density of samples annealed to $600^{\circ} \mathrm{C}^{26}$ The results show a ten times reduction in the resistivity perpendicular to the plane of the substrate (from $10^{6}$ to $10^{5} \Omega \mathrm{cm}$ ). This is a significant change, but the annealed samples are still in the range of good insulating a-tC films. The density of the films (measured by $\mathrm{x}$-ray reflectivity) also decreases with annealing from 3.0 to $2.9 \mathrm{~g} / \mathrm{cm}^{3}$. These changes are consistent with a small fraction of the 4-fold carbon converting to 3-fold carbon. Overall, the Raman, EELS, resistivity, and density measurements reveal only minor changes with annealing to temperatures near $600^{\circ} \mathrm{C}$.

\subsubsection{Stress relaxation}

We studied the stress relaxation with temperature $\left(100-600^{\circ} \mathrm{C}\right)$ and annealing time (1 - $120 \mathrm{~min}$.) of films grown at high fluence and derived a simple model for the stress relaxation. ${ }^{26}$ Samples were heated in a rapid thermal processor in an Argon ambient and the stress reduction was measured at room temperature. We report new in situ measurements of the stress

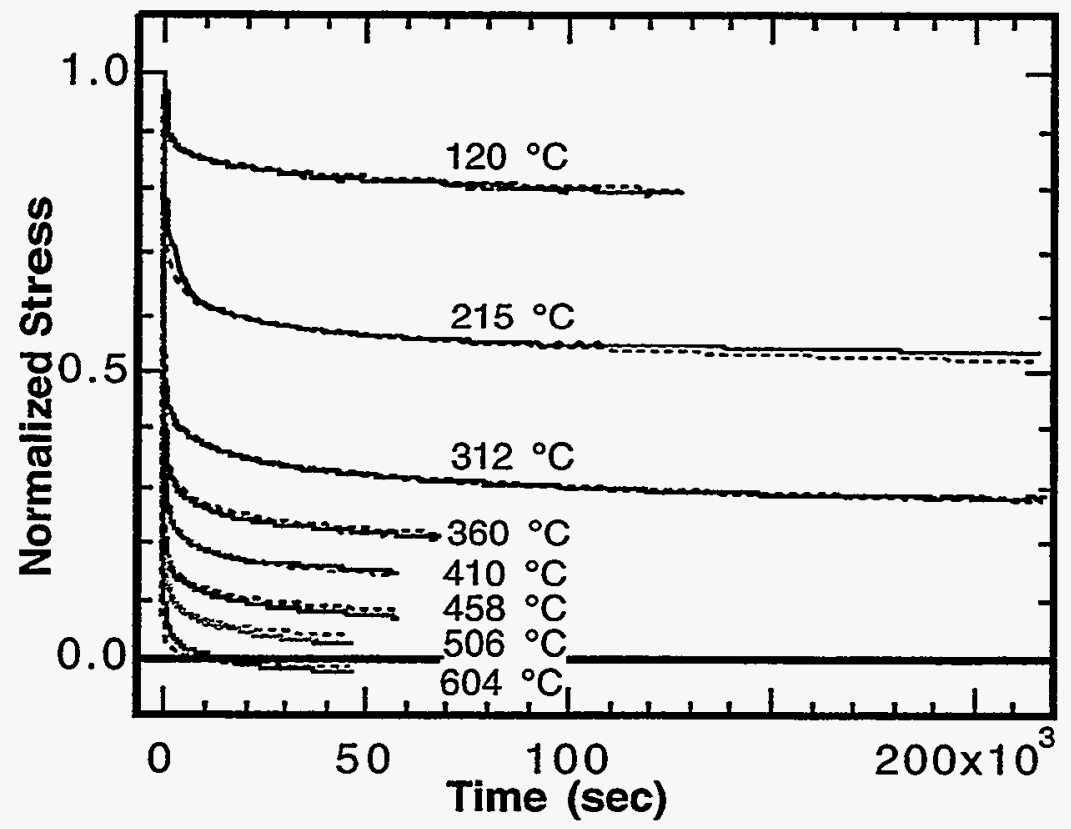

Fig. 2-13 Residual stress measured in situ as a function of annealing temperature. Each curve represents a separate sample. The dotted line represents a fit to the data as determined by equation 1 . 
relaxation for longer times (up to $60 \mathrm{hrs}$ ) and for more intermediate temperatures. Fig. 2-13 depicts the measured data which agrees well with the data reported in reference 26 . Each curve represents a separate sample, and all were grown under identical conditions. In short, the reduction in stress with annealing is quite dramatic, and irreversible, as there is only a small stress recovery upon cooling to room temperature (not shown) due to thermal mismatch. At $600^{\circ} \mathrm{C}$ the stress relaxation is quite rapid, with the bulk of the change occurring in the first few minutes of the anneal. Also, as the anneal time increases, the sign of the stress changes and the sample goes

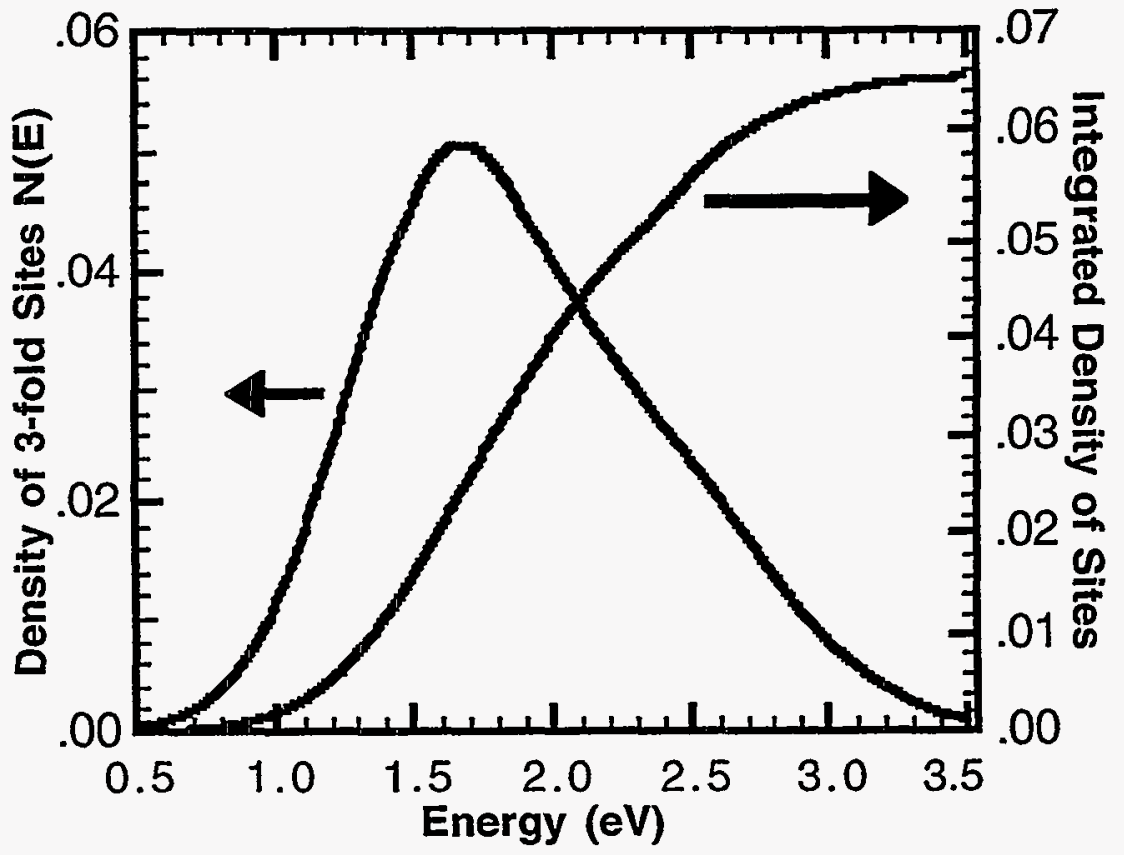

Fig. 2-14 Density and integrated density of 3-fold sites converted from 4-fold during the annealing process. into tension. We note that significant relaxation occurs at $100{ }^{\circ} \mathrm{C}$, and as the anneal temperature increases, the relaxation increases in a regular fashion that is initially quite rapid slowing with time, but never completely stopping. We note that we are the first to report detailed stress relaxation measurements with annealing in a-tC films, and that complete stress reduction has never before been reported in this materials system.

The question remains as to the nature of the stress relaxation with temperature. The data of Fig. 2-13 provides a unique opportunity to investigate potential stress reduction mechanisms. The a-tC growth process is necessarily metastable - ion bombardment of room temperature substrates. This process most certainly produces a unique amorphous microstructure due to the wide variability of carbon-carbon bonds. Indeed, theoretical simulations of the structure of a-tC films indicate that there are many strained bonds of varying length and bond angle that are far different from the usual carbon environments found in graphite, diamond, and even polymeric materials. ${ }^{27.28}$ This is supported by the low temperature $\left(100^{\circ} \mathrm{C}\right)$ at which residual stress relaxation begins, perhaps due to conversion of these metastable/strained bonds to more relaxed configurations. The Raman and EELS data imply that these rearrangements represent a small fraction of the carbon atoms in the film. Indeed, because of the high elastic modulus of this material, a small change in volume could produce a large change in stress.

We have proposed a model for the stress relaxation. ${ }^{26}$. The model proposes that the stress relaxation is a consequence of simple bond breaking processes that are induced by thermal annealing. The net result of annealing is to drive the film towards its equilibrium bonding condition which is nanocrystalline graphite (4-fold carbon is converted to 3 -fold).

The actual mechanism for the stress reduction in the films is due to the volume and shape differences between 4- and 3-fold bonded carbon. Simply stated, 4-fold carbon is roughly spherically symmetric while 3-fold carbon is more oblate. Importantly, the in-plane C-C bond of graphite is actually significantly shorter than that of diamond $(1.42 \AA$ vs. $1.54 \AA)$. A strain reduction would occur if the 3-fold bonds are preferentially oriented so that the short bonds are in the plane of the film, reducing the in-plane volume and thus the compressive stress. In addition, this would result in an overall decrease in the density of the film as the out-of-plane bond is significantly larger. This would explain the apparent reduction in biaxial stress with a 
decrease in density measured by $\mathrm{x}$-ray reflectivity.

Since the kinetics of the stress reduction will be dominated by the bond breaking process a simple first order kinetic model of bond breaking should predict $\sigma(\mathrm{t}, \mathrm{T})$, the time and temperature dependence of the stress relaxation. Equation 1 captures the essential physics. The

$$
\begin{aligned}
& \sigma(t, T)=\sigma_{0}+\frac{\varepsilon E}{1-v} \int_{0}^{\infty} N\left(E_{A}\right)\left\{1-e^{\left.-v_{0} t e^{-\left(E_{A} t_{B} T\right)}\right\} d E_{A}}\right. \\
& \sigma_{0}=\text { initial stress } \\
& \varepsilon=-0.08=\text { strain gain from conversion } \\
& E=1000 \mathrm{GPa}=\text { biaxial modulus } \\
& v=0.1 \text { Poisson's ratio } \\
& \mathrm{N}\left(\mathrm{E}_{\mathrm{A}}\right)=\text { Density of converted sites } \\
& v_{0}=10^{13}=\text { Phonon frequency }
\end{aligned}
$$

scale of the stress reduction is set by $\varepsilon$, the in-plane strain gain for a single conversion of a 4-fold to 3 -fold bond. (This number was determined by simply calculating the in-plane volume difference between a sphere of radius equal to the diamond $\mathrm{C}-\mathrm{C}$ bond distance, with an ellipse of minor radius equal to the graphite $\mathrm{C}-\mathrm{C}$ bond distance and major radius equal to the out-of-plane bond distance.) The time scale is set by a typical phonon frequency, $v_{0}=10^{13}$, and represents an attempt frequency for the conversion process. The probability of conversion is also determined by the activation barrier height which is a specific local property dependent upon the bonding condition of the carbon atom involved in the transition. In the case of a-tC the bonding configurations are highly varied, and we do not expect there to be a single energy barrier. A broad distribution would be expected. This is consistent with the stress relaxation data which shows relaxation occurring over a broad range of temperatures and time. This is represented in Eq. 1 by $N\left(E_{A}\right)$, the distribution of activation barriers. $N\left(E_{A}\right)$ is the only unknown parameter in Eg. 1. Fortunately, it can be determined by fitting the experimental stress versus time and temperature data found in Fig. 2-13.

A non-linear least squares algorithm was used to fit the data. The results are plotted as the dashed lines in Fig. 2-13. As can be seen, the data can be fit over the entire temperature range $\left(100-600^{\circ} \mathrm{C}\right)$ and from times ranging from $10-10^{5}$ seconds. The activation energy barrier distribution determined from the fit as plotted in Fig. 2-14. As expected, the distribution is quite broad ranging from $0.5-3.0 \mathrm{eV}$ in energy. The peak of the distribution is at $1.7 \mathrm{eV}$. By integrating this distribution, one can determine the number of sites converted to achieve full stress relaxation. This curve is also plotted in Fig. 2-14. It can be seen that nearly $6.5 \%$ of the 4fold sites must convert to 3 -fold in order to achieve total stress relaxation. The number of converting sites is determined by the strain gain $\varepsilon$, and if this number is not correct, the number of sites could be higher or lower. The Raman and EELS data suggest that only a small fraction of the atoms are participating in the bond rearrangements, and thus the results in Fig. 2-14 appear consistent with this result.

Using the derived $N\left(E_{A}\right)$, it is possible to calculate the number of carbon 4-fold sites converted to 3 -fold sites as a function of time and temperature. This result is simply obtained from Eq. 1 as follows:

$$
\text { Numberof converted sites }=\int_{0}^{\infty} N\left(E_{A}\right)\left\{1-e^{-v_{0} t e^{-\left(E_{A} / k_{B} T\right)}}\right\} d E_{A} \quad \text { Eq. } 2
$$

The results of these calculation are shown in Fig. 2-15. The number of sites converted as a function of time and temperature is the inverse of the stress data with an appropriate scaling factor. The time scale of the conversion process can be set by the annealing temperature. 
For fast and complete stress conversion, a temperature above $600{ }^{\circ} \mathrm{C}$ is required. For slower conversion lower temperatures can be selected, but the time scale for full conversion becomes practically infinite as the temperature approaches room temperature. For example, Eq. 1 with the derived energy barrier distribution predicts that it will take $>10^{9}$ years for total stress relaxation near room temperature. Nonetheless, Eq. 1 does predict that a-tC samples will stress relax even at room temperature. It is interesting to use the derived $N\left(E_{A}\right)$ to predict this room temperature

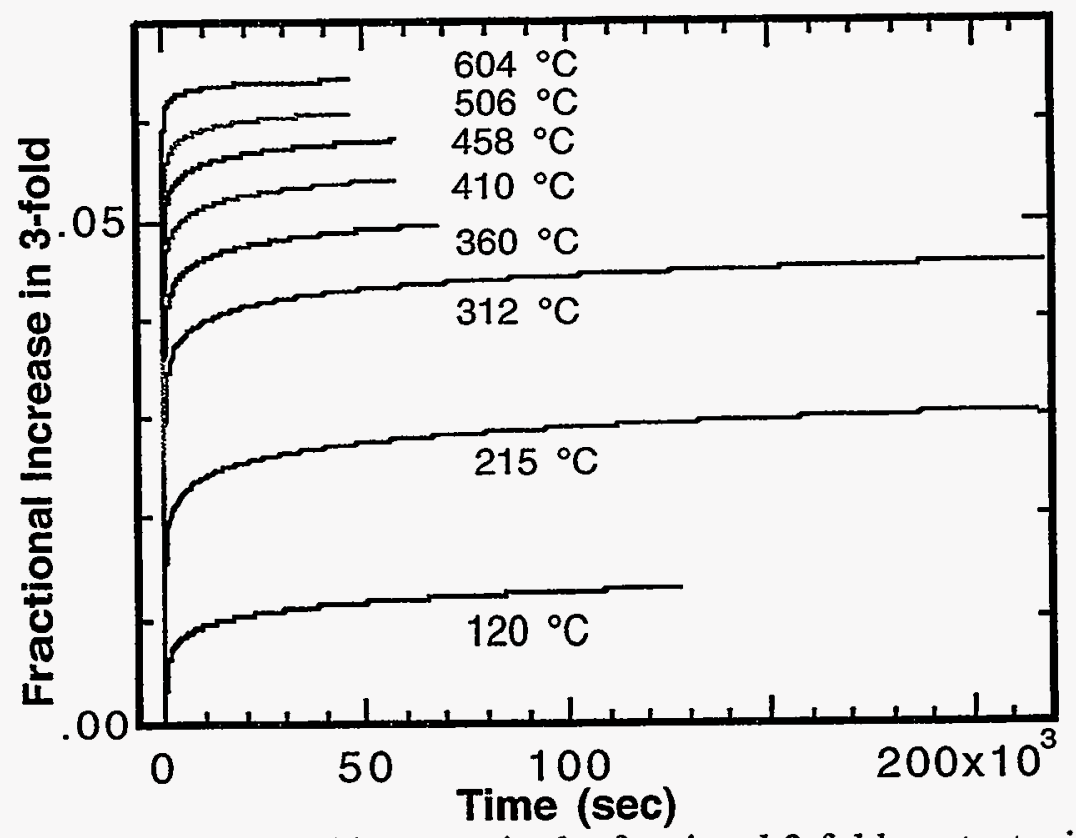

Fig. 2-15 Predicted increase in the fractional 3-fold content with annealing time from the model of Eq. 1 stress relaxation. This has been done and the result is plotted as the solid line in Fig. 2-16, where the time axis is now in units of days rather than seconds. To test this prediction, the residual stress in a series of samples were measured that had been grown $\sim 430$ days prior. The reduction in stress over this time frame is plotted as the circle in Fig. 2-16. The error bars are the average of the 10 samples measured. Within the error, the relaxation appears to be predicted by the derived energy barrier

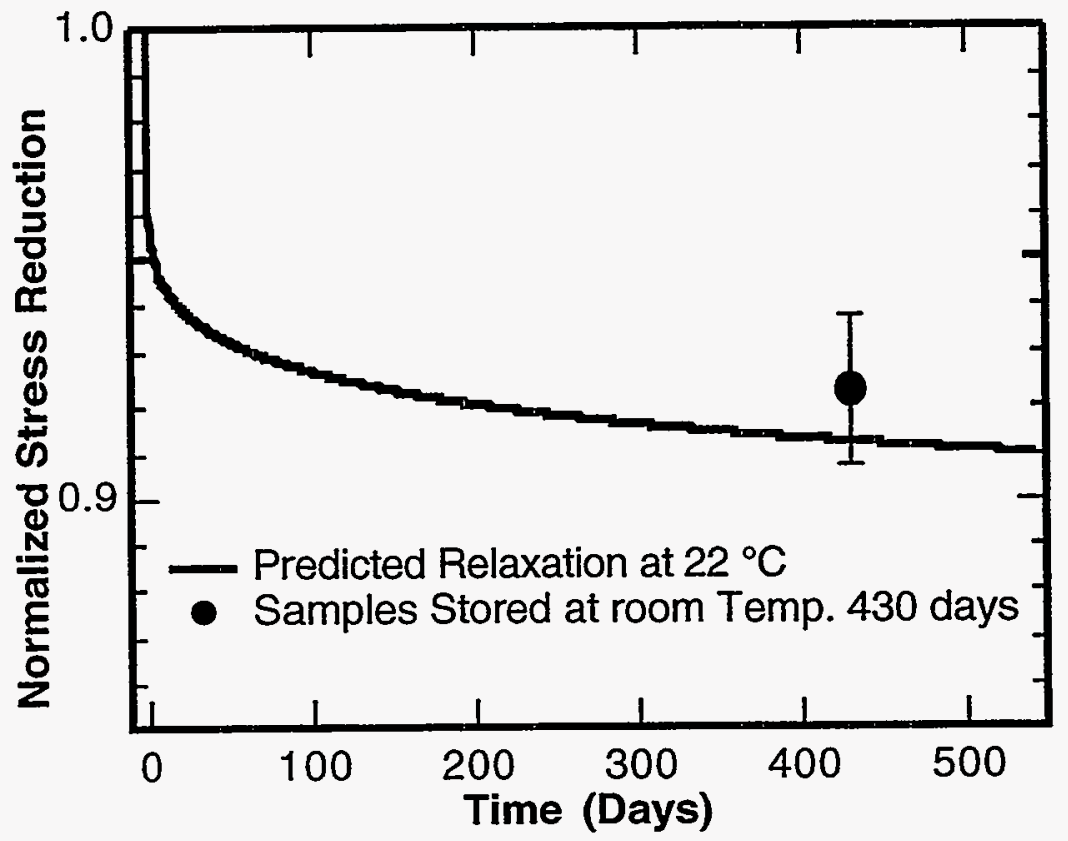

Fig. 2-16 Stress relaxation predicted at room temperature versus time in days. The data point represents the average stress reduction measured on 10 different samples that were "annealed" in air for 430 days. distribution. This implies that Eq. 1 can be used to predict the stress relaxation in a-tC samples from room temperature to $600{ }^{\circ} \mathrm{C}$ over times ranging from $10-4 \mathrm{x}$ $10^{7} \mathrm{sec}$, lending credence to the model that the stress relaxation is a direct result of the thermodynamic conversion of carbon from 4- to 3-fold configurations. It should be noted that the transformations that are occurring are definitely more complicated than simple bond conversion, in that one bond rearrangement probably leads to several other atomic rearrangements in the near vicinity. But on the average, the kinetics reduces to a simplistic description that treats this complication as a simple single atom bond breaking. 


\subsubsection{Thick stress-free multilayer samples}

Overall, the Raman and EELS measurements on thin films reveal only minor changes with, annealing, while, remarkably, $\sim 6-8$ $\mathrm{GPa}$ of residual stress is almost fully relieved. Since the annealed films are stress free with a high degree of diamond-like character, it should be possible to deposit thicker a-tC films. We tested this hypothesis by repeated growth and annealing steps. First, we grew a film in vacuum $(1500-2000 \AA$

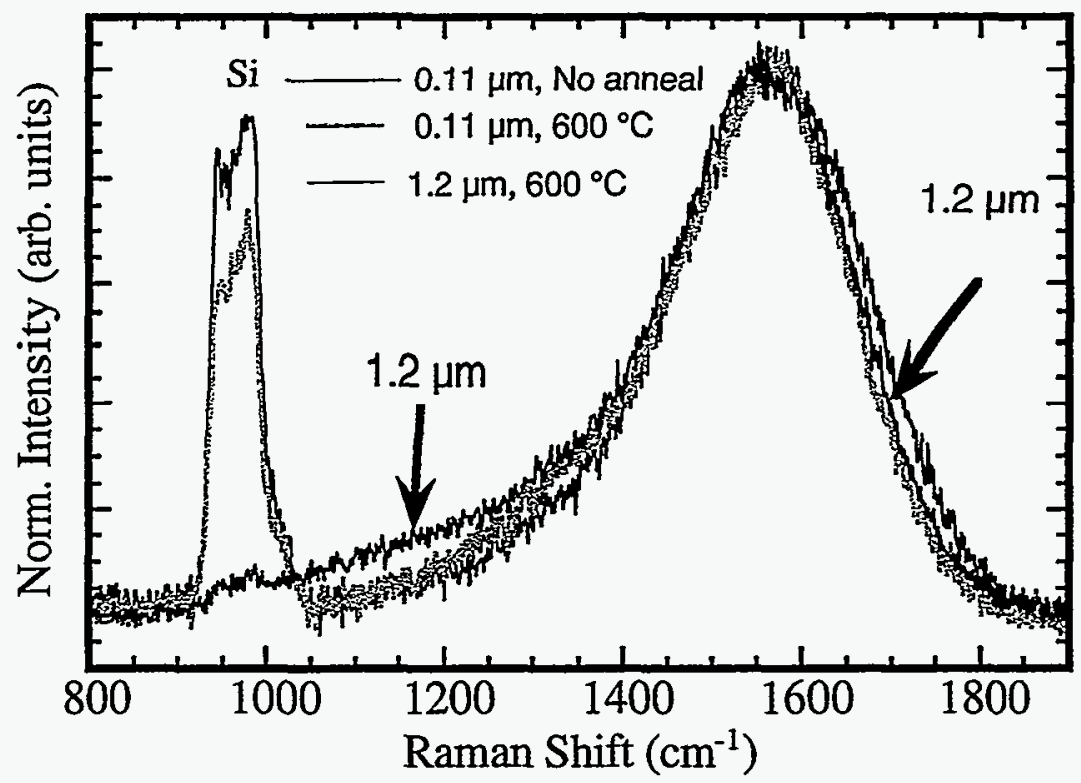

Fig.2-17 Raman spectrum of single-layer as-grown, singlelayer annealed, and six-layer annealed thick a-tC films.

thick) at room temperature and high laser energy density (conditions that produce high quality atC films with high stress). Next, we heated the sample in situ to $600{ }^{\circ} \mathrm{C}$ for 2 minutes, followed by a cooling to room temperature, where the deposition and annealing procedure was repeated. In this manner, we grew several thick films; the thickest was $1.2 \mu \mathrm{m}$ thick. From the change in wafer curvature, this thick film had $0.2 \mathrm{GPa}$ of residual compressive stress. (A film grown to $0.92 \mu \mathrm{m}$ thickness by multiple growth and annealing steps showed no change in wafer curvature after deposition.) The thick films were all adherent to the substrate and showed no signs of spalling. In principle, we should be able to grow films to arbitrary thicknesses. Indeed, to date we have grown a-tC films up to $10 \mu \mathrm{m}$ thick.

Fig. 2-17 depicts Raman spectra of single-layer as-grown, single-layer annealed $\left(600^{\circ} \mathrm{C}\right)$, and six-layer annealed $\left(600^{\circ} \mathrm{C}\right)$ films. The Raman spectrum of the thin annealed film is very similar to that of the unannealed thin sample. For the thick specimen there are greater changes. The peak at $965 \mathrm{~cm}^{-1}$ from the Si substrate is much reduced in intensity indicating increased opacity, and there is greater intensity in the $1000-1400 \mathrm{~cm}^{-1}$ region consistent with a fractional increase in the 3-fold carbon content. Visually, the film displays color "fringes" as well, indicating some transparency.

We used a NanoIndenter II ${ }^{\mathrm{TM}}$ to measure the hardness of these thick films. With thick a-tC films, the substrate effects are reduced compared to thin films, and thus the data should be more representative of the true film properties. For all depths probed, the force vs. depth curves (inset of fig. $2-18)$ revealed a high degree of elastic recovery $(\sim 85 \%)$ following indentation typical of very hard carbon coatings. ${ }^{16,29.30}$

Fig. 2-18 shows the hardness and nanoindentation elastic modulus of a $1.2 \mu \mathrm{m}$ thick a-tC film as determined from the loading/unloading curves using the Pharr-Oliver ${ }^{31}$ method. Each point represents the average of ten separate indents, and the error bars are calculated from the standard deviation of these ten results. The film hardness for shallow indents $(<100 \mathrm{~nm})$ is above $95 \mathrm{GPa}$. As the indent depth increases, the hardness initially quickly decreases till $\sim 150$ $\mathrm{nm}$, then falls again but with a shallower slope. The elastic modulus of the film starts near 750 $\mathrm{GPa}$ for the $70 \mathrm{~nm}$ indents and falls approximately linearly with indent depth. For shallow indents $(<100 \mathrm{~nm})$, the indenter tip shape must be well calibrated and the plastic deformation of the material accounted for accurately. Data for the deeper indents are probably affected by yield of the substrate or compliance of the indenter/substrate column as high forces, $>60 \mathrm{mN}$, are needed to reach $300 \mathrm{~nm}$ depths. The modulus values are also probably low due to the high 


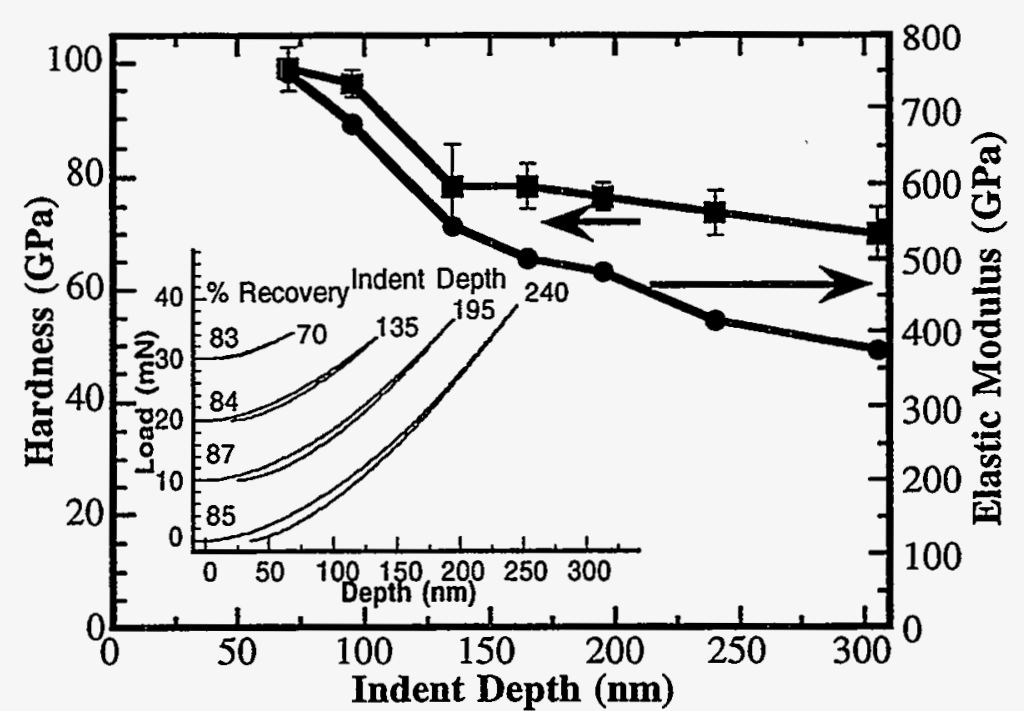

Fig. 2-18 Hardness and nanoindentation elastic modulus vs. depth of indent for a $1.2 \mu \mathrm{m}$ thick a-tC film. Inset shows force vs. depth curves (offset for clarity).

degree of elastic recovery and substrate effects. Unfortunately, even for these thick films, substrate effects appear to limit the accuracy of the indentation analysis. Another concern for films of such high apparent hardness is yielding of the diamond indenter tip.

To increase confidence in the hardness measurement, we performed another set of indentations on the same sample to a fixed depth of $0.15 \mu \mathrm{m}$ on a second indenter (Nano Instruments $^{\mathrm{TM}}$ ). To check for deformation of the tip, a new tip was selected for the experiments and the area function measured using indents into fused silica. A series of indents into silica was performed before and after examining the a-tC film, with no change in area function observed. In addition, the a-tC sample was indented 10 times, and the curves were very reproducible; the spread between indents of measured force at $150 \mathrm{~nm}$ is $<0.05 \mathrm{mN}$, with no discernible trend with time sequence. This reproducibility in area function and indent data implies the diamond indenter is not plastically deforming. The values of hardness and modulus determined directly from the data were 80.2 $\mathrm{GPa}$ and $552 \mathrm{GPa}$, respectively in good agreement with the data from Fig. 2-18.

To deconvolve the effects of the "soft" substrate, a finite-element modeling technique ${ }^{32.33}$ was used to fit the data from Nano Instruments. The two parameters for the a-tC layer which are extracted from the fit of the simulation to experimental force versus depth response are the yield stress (defined at a plastic strain of .002) and Young's modulus. Hardness is deduced by an

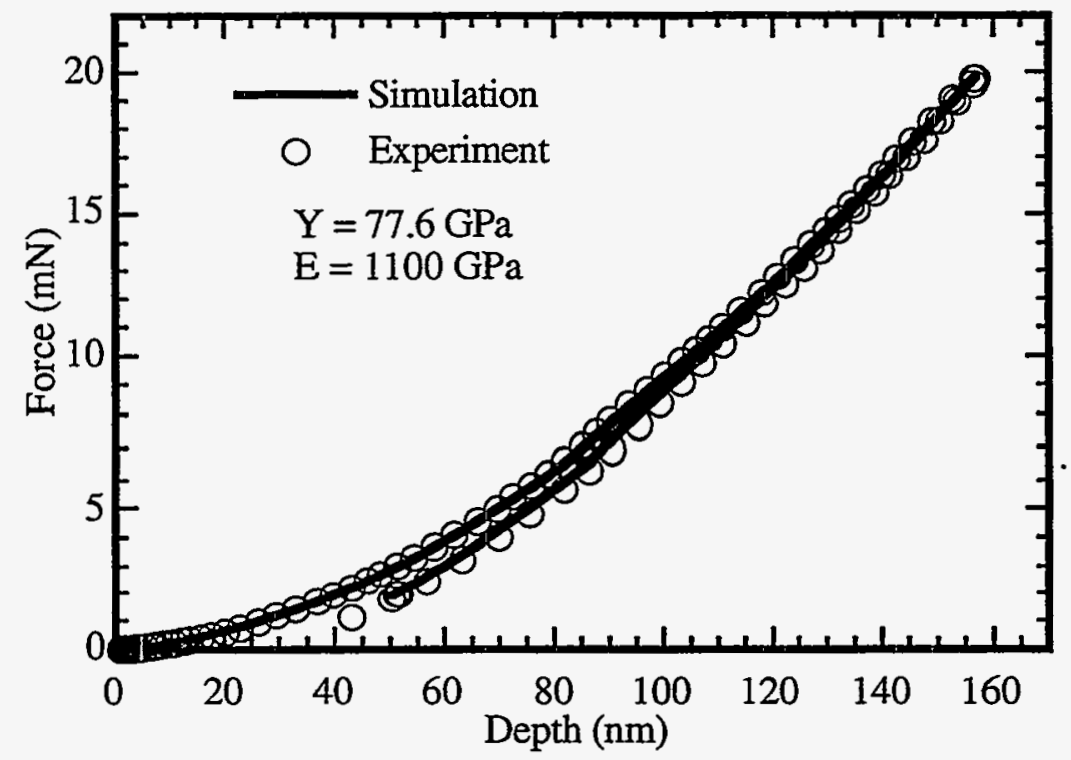

Fig.2-19. Experimental nanoindentation force vs. depth curve and best-fit simulation for a $1.2 \mu \mathrm{m}$ a-tC layer on Si. Hardness is deduced by a separate simulation of a "bulk" sample. additional simulation using the yield stress and elasticity of the layer in a hypothetical bulk "sample". Since no yielding of the indenter tip was observed in the experimental data, the simulated tip was not allowed to plastically deform.

Fig. 2-19 shows a typical experimental nanoindentation curve and the best-fit simulation for the $1.2 \mu \mathrm{m}$ layer a-tC film on Si. The best fit was obtained with a yield stress of $77.6 \pm 2.4 \mathrm{GPa}$ and a Young's modulus of $1100 \pm 18$ $\mathrm{GPa}$ for the layer material. A measured pre-existing compressive stress of $0.2 \mathrm{GPa}$ was included in the simulation. The separate simulation of a "bulk" sample 
of this material gave a hardness of $88.1 \pm 2.0 \mathrm{GPa}$ (see Table I). The commonly observed factor of $\sim 3$ ratio of hardness to yield stress is not observed since this is a non-metallic material with no work hardening. For this relatively thick film the substrate has about a $10 \%$ effect on the apparent hardness but a 50\% effect on the apparent elasticity.

Table 1. Materials properties deduced by nanoindentation and modeling of $150 \mathrm{~nm}$ indents into a $1.2 \mu \mathrm{m}$ thick a-tC film. Values for crystalline diamond are listed for reference.

\begin{tabular}{cccc}
\hline & $\begin{array}{c}\text { Yield Stress } \\
(\mathrm{GPa})\end{array}$ & $\begin{array}{c}\text { Young's Modulus } \\
(\mathrm{GPa})\end{array}$ & $\begin{array}{c}\text { Hardness } \\
(\mathrm{GPa})\end{array}$ \\
\hline Experiment & & 550 & 80 \\
\hline Simulation & $77.6 \pm 2.4$ & $1100 \pm 18$ & $88.1 \pm 2.0$ \\
\hline Diamond & & 1140 & 100 \\
\hline
\end{tabular}

The hardness of $88.1 \mathrm{GPa}$ for the hardest film can be compared to an expected hardness for the diamond indenter of $\sim 100 \mathrm{GPa}^{34}$ Examination of the calculated stresses in the indenter material show a peak value of $106 \mathrm{GPa}$ in the center of the indenter $-50 \mathrm{~nm}$ behind the tip. A series of simulations we did on somewhat softer films gave a result of $\sim 75 \mathrm{GPa}^{33}$ We thus can increase the lower bound on the compressive yield strength of crystalline diamond to $-100 \mathrm{GPa}$.

In conclusion, the compressive stress in a-tC films can be relieved by short thermal anneals near $600^{\circ} \mathrm{C}$. Raman and EELS spectra of annealed films show only subtle changes indicating the films retain their diamond-like nature. This knowledge has been used to grow thick a-tC films $(>1.2 \mu \mathrm{m})$ that are stress-free and adherent. Nano-indentation and finite-element modeling have been combined to show these films have very high hardness ( $88 \mathrm{GPa}$ ) and modulus (1100 $\mathrm{GPa}$ ), near the reported values of bulk diamond. Also, a lower bound on the compressive yield stress of bulk diamond ( $100 \mathrm{GPa})$ has been extracted from the analysis. Finally, stress may be intrinsic to the a-tC growth process, but it is not necessary for films with extreme hardness.

\subsubsection{CONCLUSIONS}

Characterization of diamond-like carbon films has allowed us to understand the changes in film structure as they relate to the deposition conditions. In short, the film properties are controlled by the laser fluence on the target. Low fluences lead to low energy ablated species that produce carbon films that are largely low density and graphitic. High fluences lead to more energetic species that produce films with relatively high density and diamond-like properties. Unfortunately, the residual stress in the film rises dramatically with laser fluence. The residual stress appears to be intrinsic to the growth of a-tC films.

Although a-tC films are produced at room temperature, they are remarkably stable up to annealing temperatures of $800^{\circ} \mathrm{C}$. Changes in the structure of these films are minor, yet remarkably, the residual stress can be completely relieved.

Large residual stresses in diamond-like carbon films have been a major limitation preventing their use in many applications. In particular, these stresses have prevented the deposition of thick films and the production of free-standing membranes. The work funded in this LDRD has allowed lead to a solution of this problem. Namely, production of thick films by deposition of multilayers where the film is built up by repeated deposition and annealing steps. 


\subsection{Finite-element modeling of nanoindentation for evaluating mechanical properties of}

\section{the amorphous-tetrahedral carbon films}

Thin films of very hard materials such as amorphous tetrahedrally-bonded carbon $(a-t C)$ have many important applications, but characterizing their mechanical properties becomes more difficult with both increasing hardness and decreasing thickness. The most commonly used technique is depth-sensing indentation. ${ }^{35-38}$ Although the measurement itself is very precise, mechanical properties are generally extracted from the data using an analytical solution with a number of simplifying assumptions. ${ }^{37-40}$ For most bulk materials and relatively thick films this approach works very well, but for thin films obtaining a substrateindependent determination of the properties becomes increasingly difficult. For materials approaching the hardness of bulk diamond as were produced in this project, deformation and possible yielding of the indenter tip are additional complications. Since an analytical solution is not feasible for such cases, we used an analysis approach using finite-element modeling to extract mechanical properties. ${ }^{33.41-}$

To test the

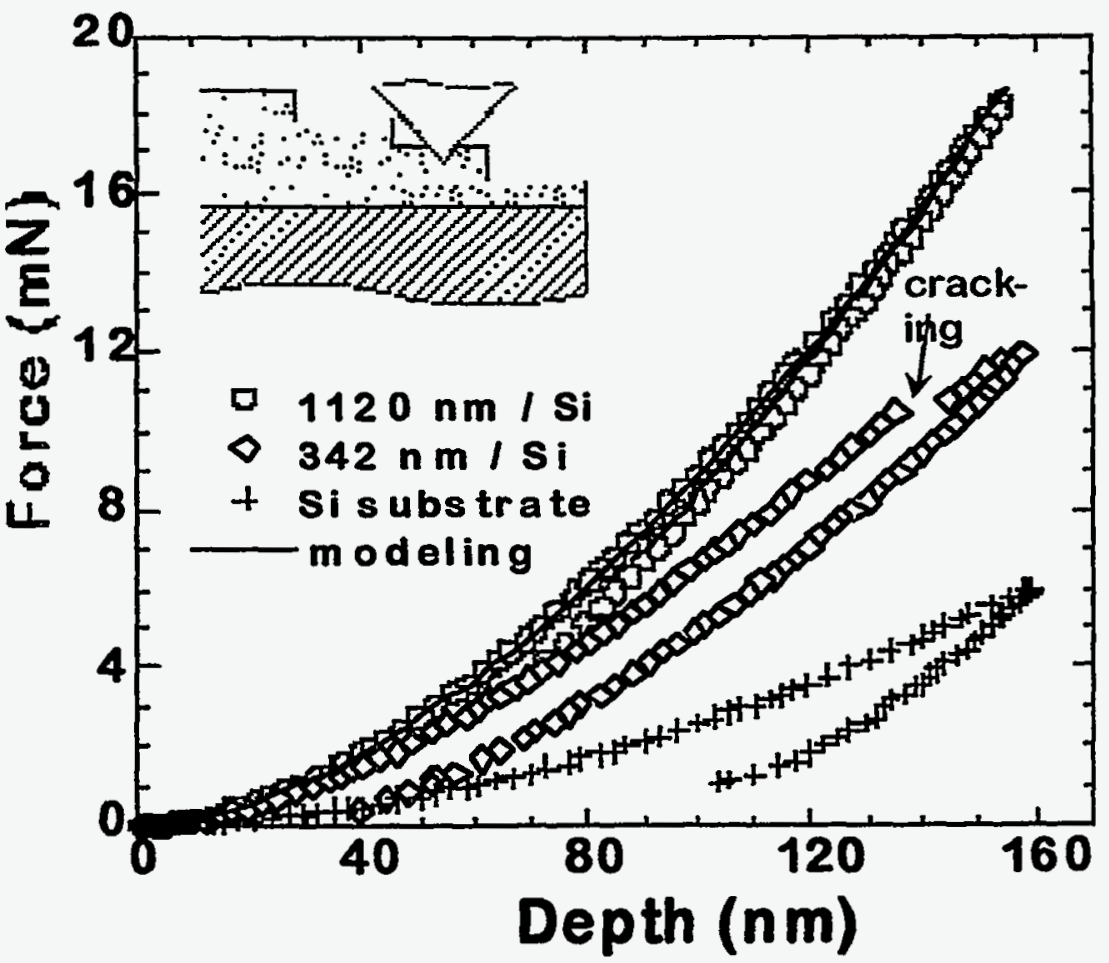

Fig. 2-20 Nanoindentation load-displacement curves obtained from two thicknesses of $a-t \mathrm{C}$ on Si and from bare Si. The solid line is a best-fit simulation to the $1120 \mathrm{~nm}$ layer data. capabilities of the modeling-based analysis and to compare results with conventional analyses of hard films, samples of $a-t C$ were grown on a Si substrate by pulsed laser deposition, with a range of thicknesses, using methods described elsewhere in this report. Sequential layers were deposited, with each layer annealed in situ to $600^{\circ} \mathrm{C}$ before another layer was deposited. As discussed earlier, the annealing relieves the compressive stress typical of deposited $a-t C$ and permits very thick, stress-free films to be built up. A movable mask was used to construct a step structure with total thicknesses of $48,152,342$, $514,716,928$ and $1120 \mathrm{~nm}$. Each step height was measured by a profilometer and then indentation testing was performed on each separate area.

Figure 2-20 shows conventional load-displacement curves obtained from the 342 and $1120 \mathrm{~nm} a-t C$ thicknesses, as well as from the Si substrate alone, while the solid line is from the modeling analysis. Qualitatively, the load-displacement curves demonstrate that the $a-t C$ layers are much harder than the Si and show little plastic deformation. However, the curve for $342 \mathrm{~nm}$ $a-t \mathrm{C}$ shows a discontinuity at $140 \mathrm{~nm}$ displacement due to cracking of the layer. Since any analysis requires both force and contact stiffness, which for a conventional indent is only determined at the start of unloading, cracking renders the data useless for analysis. To measure properties at this thickness or less, we used the Continuous Stiffness Measurement (CSM), a 
dynamic nanoindentation test with an AC modulation imposed on the indenter position during loading. The frequency response then gives the stiffness for all depths. Figure 2-21 shows force and stiffness for three different thicknesses of $a-t C, 152,342$, and $1120 \mathrm{~nm}$. The solid lines are modeling fits. Cracking is again evident in Fig. 2-21(a) near $140 \mathrm{~nm}$ for the data from $342 \mathrm{~nm}$ and near $55 \mathrm{~nm}$ for the $152 \mathrm{~nm}$ film, but since stiffness is now available at all depths, the data up to the point

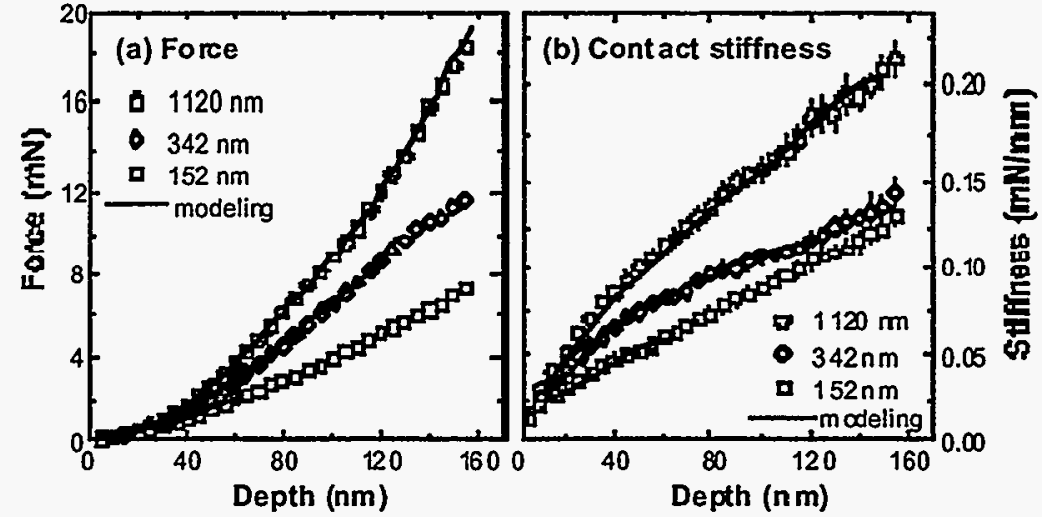

Fig.2-21. Nanoindentation force (a) and contact stiffness (b) for three thicknesses of $a-t C$ on Si by CSM. Solid lines are best-fit simulations. of cracking can be used for analysis.

In what we will refer to as the conventional analysis, the measured values of force $F$ and stiffness $S$ are used to derive first the projected area of contact and then the hardness $\mathrm{H}$ and Young's modulus of elasticity $\mathrm{E}$. The depth of contact between the indenter and the material is calculated by the Oliver-Pharr method, ${ }^{37}$ and then the projected area $A$ of contact is evaluated using the cross-sectional shape of the indenter, generally calibrated by indenting a known sample and iteratively fitting the results. Hardness is then $\mathrm{H}=\mathrm{F} / \mathrm{A}$, here defined using the projected area of contact under load. $\mathrm{E}$ is determined from $\mathrm{A}$ and $\mathrm{S}$ using the following equation, based on Sneddon's solution ${ }^{39,40}$ of the elastic contact between a rigid, axisymmetric punch and an elastic half space:

$$
E_{r}=\left[\frac{\left(1-v^{2}\right)}{E}+\frac{\left(1-v_{i}^{2}\right)}{E_{i}}\right]^{-1}=\frac{1}{\beta} \frac{\sqrt{\pi}}{2} \frac{S}{\sqrt{A}}
$$

where $\beta=1.034$ for a Berkovich tip ${ }^{45}$ and where $E$ and $v$ and $E_{i}$ and $v_{i}$ are Young's modulus and Poisson's ratio for the sample and the diamond indenter, respectively. The derivation of Eq. 3
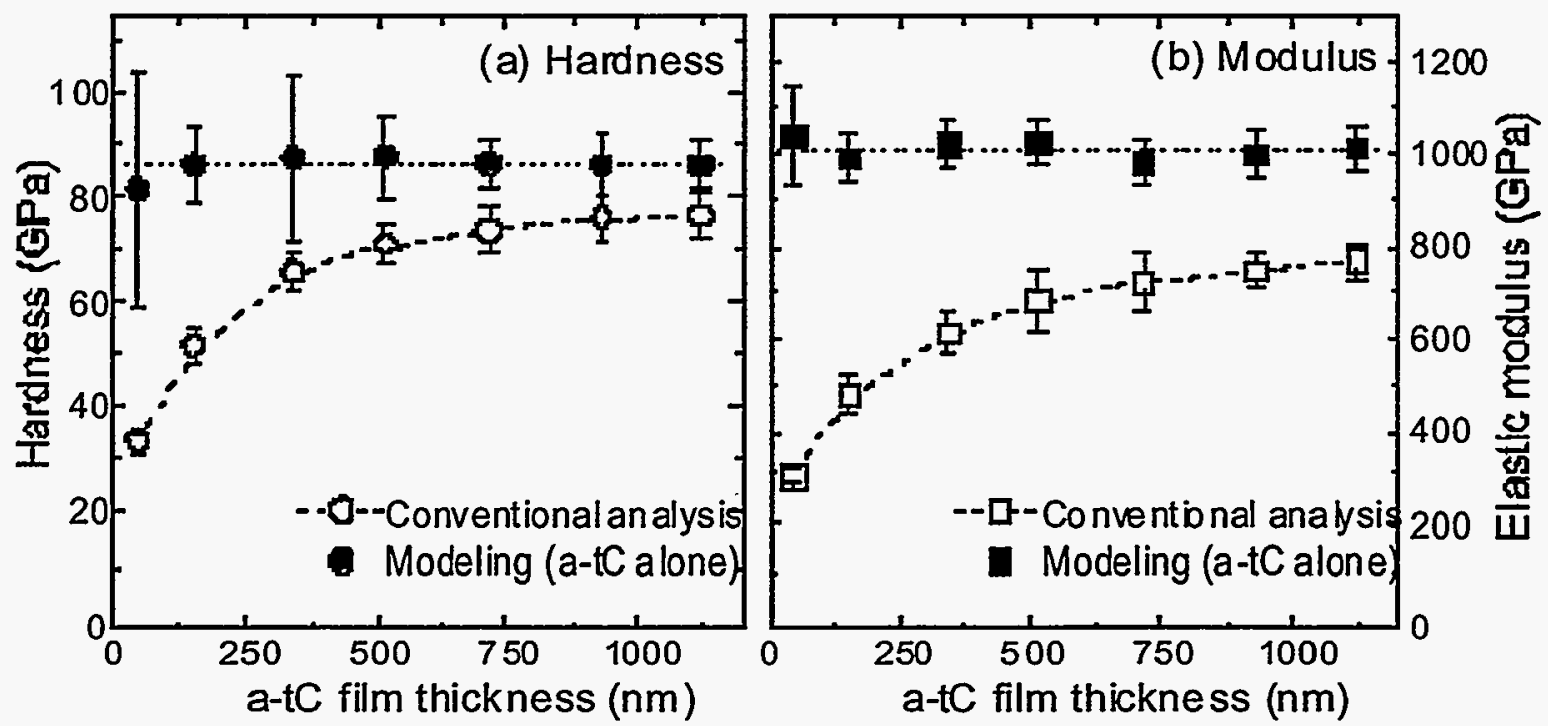

Fig.2-22. Hardness and elastic modulus determined for each $a$ - $t \mathrm{C}$ layer thickness by the conventional analysis and by the modeling analysis. 


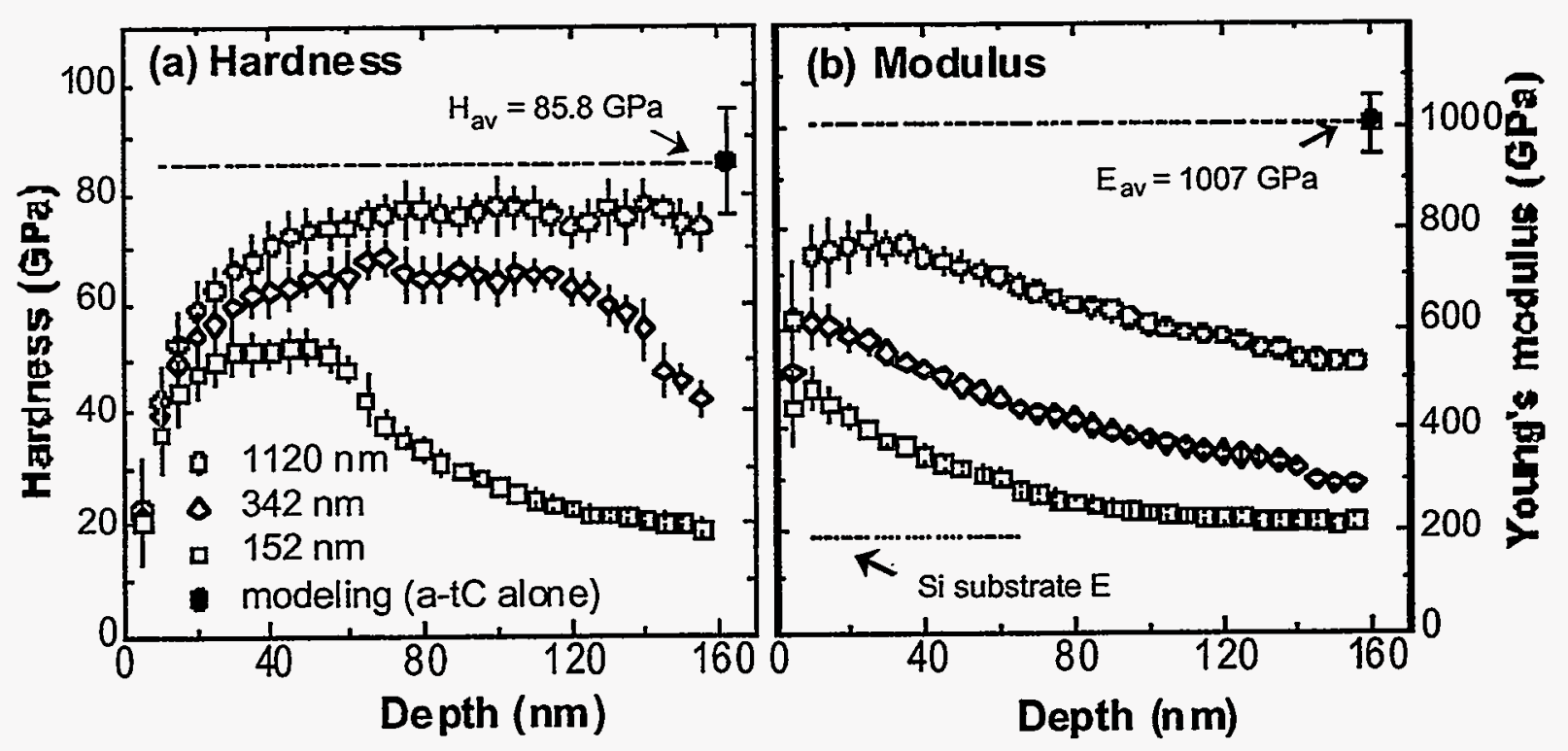

Fig.2-23. (a) Hardness vs. displacement derived from the CSM data of Fig. 2-21 by a conventional analysis. Dashed line is the average $a-t C$ hardness derived by the modeling analysis. (b) Elastic modulus vs. displacement from the conventional analysis. Dashed line is the average, $a-t C$. modulus derived hv the modeling analvsis. uses several assumptions, but the most important is that of a rigid indenter. To account for the elastic response of the indenter, Eq. 1 is defined using a reduced modulus $E_{r}$, as shown.

Applying the conventional analysis to the data obtained from the $1120 \mathrm{~nm}$ thick $a-t \mathrm{C}$ film on $\mathrm{Si}$ in Fig. 2-20 gives $74 \pm 4 \mathrm{GPa}$ for the hardness and $533 \pm 12 \mathrm{GPa}$ for the modulus, assuming $\mathrm{E}_{\mathrm{i}}=1140 \mathrm{GPa}$ and $v_{i}=0.07$, and $v=0.1$ for the $a-t \mathrm{C}$. When the analysis is applied to the CSM data in Fig. 2-21, both $\mathrm{H}$ and $\mathrm{E}$ are obtained as a function of depth, as shown in Fig. 2-23. The effects of cracking in the two thinner films are again seen near 55 and $140 \mathrm{~nm}$. Figure 2-24 summarizes the results of the conventional analysis with open symbols; the peak value obtained is shown for each thickness. It is clear from Figs. 2-23 and 2-24 that the values of $\mathrm{H}$ and $\mathrm{E}$ derived by the conventional method have not converged with increasing film thickness, even for the $1120 \mathrm{~nm}$ film. The apparent modulus, in particular, has not plateaued with film thickness and also falls off rapidly for each film with indent depth, approaching the value for the Si substrate. Both $H$ and $E$ derived by the conventional analysis are composite values, since the experimental force and stiffness are due to the combined properties of the film and substrate. It is often assumed that using an indent depth $<10 \%$ of the layer thickness minimizes contributions of the substrate, allowing the layer properties to be determined by the conventional analysis. For these $a-t C$ films, however, the substrate is clearly influencing the analysis even for the thickest film.

The modeling-based analysis we developed. ${ }^{33,41-44}$ uses the commercial, large-strain, finite-element code ABAQUS/Standard ${ }^{46}$ to simulate the indentation experiments. Separate utilities are used to generate meshes and depth-dependent material descriptions specific to each sample and to fit the results. Blunting of the indenter tip, friction between tip and surface, and pre-existing stress in the layers are all included in the modeling. We extract the yield strength $\mathrm{Y}$, defined as the yield at a plastic strain of 0.002 , and $E$ intrinsic to the layer material from the fit of simulation to experiment. An additional simulation using these values to define a hypothetical bulk "sample" subsequently deduces the intrinsic H of the layer material. This intrinsic H can then be compared directly to measurements of bulk materials or to other layers of differing thicknesses or layers on other substrates.

A two-dimensional axisymmetric mesh was constructed for each sample, which includes the shape (area function) of the indenter. Details of the typical mesh parameters are given elsewhere ${ }^{44}$, but for modeling the $a-t C$ films it was especially important to make the portion of 
the mesh describing the indenter as large as the sample itself, since the diamond indenter can deform during the measurement nearly as much as the sample. All materials were modeled as isotropic, elastic-plastic solids, using the classical plasticity model in ABAQUS with a Mises yield surface and associated plastic flow. ${ }^{47}$ This and the use of the two-dimensional mesh are the main simplifying assumptions for the modeling. A density, Poisson's ratio $\mathrm{V}$, and stress-strain curve were specified for each material, with the stress-strain curve parameterized by $E$ and $Y$. Work hardening was assumed to be absent for both the $a-t \mathrm{C}$ films and Si substrate. The Si substrate was modeled with $\mathrm{Y}=5.65 \mathrm{GPa}$ and $\mathrm{E}=185 \mathrm{GPa}$, determined by separately fitting indentation of the bare substrate. We assumed $v=0.10$ for the $a$ - $t C$ material, while the diamond in the indenter was modeled using $v_{i}=0.07, E_{i}=1140 \mathrm{GPa}$ and $Y_{i}=100 \mathrm{GPa}$. Comparison of standard indents into silica before and after indenting the $a-t C$ layers showed that the diamond indenter did not permanently deform during these experiments, so the calculated peak stresses of $\sim 100 \mathrm{GPa}$ within the tip during these indents represent a lower bound on the yield strength of diamond under conditions of hydrostatic compression.

To deduce $\mathrm{Y}$ and $\mathrm{E}$ for each $a-t \mathrm{C}$ layer, a series of simulations were used, each with slightly different properties for the unknown material. Calculated forces and stiffnesses were compared to observed values and an interpolation made to optimal values of $\mathrm{Y}$ and $E .{ }^{45}$ Once a simulated load-displacement curve was obtained that gave a "best fit" to the experimental curve within the errors, the $Y$ and $E$ were taken to be those of the unknown material, with error bars estimated from both experimental errors and interpolation parameters. A similar procedure was used to fit the data from the CSM experiments, with the stiffness as a function of depth derived for each simulation using short unloading steps at regular depth intervals. ${ }^{44}$

The solid line in Fig. 2-20 shows the best-fit simulation obtained for the $1120 \mathrm{~nm} a-t C$ sample, with $\mathrm{Y}=69 \mathrm{GPa}$ and $\mathrm{E}=1008 \mathrm{GPa}$ for the $a-t \mathrm{C}$. The best-fit simulations for the three sets of CSM data in Fig. 2-21 are also shown as solid lines for each force and stiffness curve, with only the data obtained before cracking used for the two thinner films. The average $Y$ calculated by modeling of all thicknesses was $68.2 \pm 12 \mathrm{GPa}$. The average intrinsic hardness, $85.8 \pm 10 \mathrm{GPa}$, is plotted in Fig. 2-23(a) as a dashed line for comparison to the values derived by the conventional method. Figure 2-24(a) shows the hardness derived for each layer thickness with solid circles. The larger error bars for the thinnest films are the consequence of a small fraction of plastic flow during the indents, which leads to a high uncertainty in Y. For reference, the hardness of crystalline diamond is generally accepted as $\sim 100 \mathrm{GPa} .{ }^{48}$ In a similar way, Fig. 223(b) shows the average E for all layers, $1007 \pm 58 \mathrm{GPa}$, and Fig. 2-24(b) shows the E deduced for each thickness with solid squares. The consistency of the derived values for both $\mathrm{H}$ and $\mathrm{E}$ for all film thicknesses indicates the accuracy of the analysis method and confirms that the $a-t C$ mechanical properties are being determined independently of the substrate.

Figure 2-24 also confirms that the conventional analysis is giving incorrect values for $\mathrm{H}$ and E, even for the thickest film at $1120 \mathrm{~nm}$. There appear to be two primary reasons for this. First, an important approximation in the conventional analysis is that Sneddon's solution for a rigid indenter can be used, with a reduced modulus accounting for any elastic deformation. For most materials this works very well, but for very hard materials such as $a-t C$, deformation of the indenter can be substantial, and the modeling shows that the approximation is inappropriate. For example, the calculated compression of the indenter tip during these experiments is as much as $60 \mathrm{~nm}$ at a $160 \mathrm{~nm}$ indent depth, significantly altering its shape. Second, values derived for both $\mathrm{H}$ and $\mathrm{E}$ depend critically on a correct determination of the area of contact, which is indirectly found using contact stiffness. ${ }^{37}$ For a hard film on a much softer substrate, such as here, "sinkin" of the layer into the substrate ${ }^{49}$ strongly affects stiffness and the contact area determination, even for a relatively thick film.

These modeling results have implications for indentation testing of all very hard materials, both thin layers and bulk. For very hard layers on softer materials, the modeling has shown that sink-in is a severe problem. Furthermore, deformation of the indenter when testing such hard materials is even more of a concern for bulk samples, and is an effect that can only be accounted for by modeling. Since a Sneddon-based analysis of indentation load-displacement data uses the 
assumption of a rigid indenter, the significant deformations we calculate appear to be a fundamental problem. Finite-element modeling appears to be the only way to accurately deduce the hardness and modulus of a material that approaches the hardness of diamond.

\subsection{References}

1J. P. Sullivan, T. A. Friedmann, and A. G. Baca, J. Electr. Mater. 26, 1021 (1997).

2 G. S. Higashi, Y. J. Chabal, G. W. Trucks, and Krishnan Raghavachari, Appl. Phys. Lett. 56, 656 (1990).

3M. Ramsteiner and J. Wagner, Appl. Phys. Lett. 51 (17), 1355 (1987).

${ }^{4}$ M. F. Toney and Sean Brennan, J. Appl. Phys. 66, 1861 (1989).

5C. A. Lucan, T. D. Nguyen, and J. B. Kortright, Appl. Phys. Lett. 2, 101 (1991).

6Y. Huai, M. Chaker, J. N. Broughton, E. Gat, and H. Pepin, Appl. Phys. Lett. 65, 830 (1994).

$7 \mathrm{~J}$. Als-Nielsen, in Structure and Dynamics of Surfaces, edited by W. Schommers and P. von

Blanckenhagen (Springer, Berlin, 1987), Vol. II, pp. 181.

$8 \mathrm{M}$. Born and E. Wolf, Principles of Optics (Pergamon, Oxford, 1970).

${ }^{9}$ G. G. Stoney, Proc. R. Soc. London, Ser. A 82, 172 (1909).

10 E. Chason, J. A. Floro, T. M. Mayer, B. K. Kellerman, and D. P. Adams, Abstracts of Papers

of the American Chemical Society 213 (pt.2), 33 (1997).

11P. T. Murray and D. T. Peeler, Appl. Surf. Sci. 69, 225 (1993).

12 Y. Lifshitz, G. D. Lempert, E. Growssman, I. Avigal, C. Uzan-Sagay, R. Kalish, J. Kulik, D. Marton, and J. W. Rabalais, Diamond Relat. Mater. 4 (4), 318 (1995).

${ }_{13}$ H. Hofsäss, H. Binder, T. Klumpp, and E. Recknagel, Diam. Rel. Mater. 3, 137 (1993).

14J. J. Cuomo, D. L. Pappas, J. Bruley, J. P. Doyle, and K. L. Saenger, J. Appl. Phys. 70 (3), 1706 (1991).

15D. R. McKenzie, D. Muller, B. A. Pailthorpe, Z. H. Wang, E. Kravtchinskaia, D. Segal, P. B. Lukins, P. D. Swift, P. J. Martin, G. Amaratunga, P. H. Gaskell, and A. Saeed, Diam. Rel. Mater. 1,51 (1991).

$1{ }^{16}$ G. M. Pharr, D. L. Callahan, S. D. McAdams, T. Y. Tsui, S. Anders, A. Anders, J. W. Ager III, I. G. Brown, C. S. Bhatia, S. R. P. Silvia, and J. Robertson, Appl. Phys. Lett. 68 (5), 779 (1996).

17P. J. Fallon, V. S. Veerasamy, C. A. Davis, J. Robertson, G. A. J. Amaratunga, W. I. Milne, and J. Koskinen, Phys. Rev. B 48 (7), 4777 (1993).

18D. R. McKenzie, D. Muller, and B. A. Pailthorpe, Phys. Rev. Lett. 67 (6), 773 (1991).

19 Y. Lifshitz, G. D. Lempert, and E. Grossman, Phys. Rev. Lett. 72 (17), 2753 (1994).

20P. B. Mirkarimi, K. F. McCarty, and D. L. Medlin, Mater. Sci. Eng. R-Reports 71 (26), 3820 (1997).

21J. W. Ager III, Simone Anders, Andre Anders, and Ian G. Brown, Appl. Phys. Lett. 66 (25), 3444 (1995).

22 T. E. Doyle and J. R. Dennison, Phys. Rev. B 51 (1), 196 (1995).

${ }^{23}$ D. R. Tallant, T. A. Friedmann, N. Missert, M. P. Siegal, and J. P. Sullivan, Mat. Res. Soc. Proc 498, 37 (1998).

24D. R. Tallant, Private communication .

25 T. A. Friedmann, K. F. McCarty, J. C. Barbour, M. P. Siegal, and Dean C. Dibble, Appl. Phys. Lett. 68 (12), 1643 (1995).

26J. P. Sullivan, T. A. Friedmann, D. R. Tallant, J. Mikkalson, D. J. Rieger, A. G. Baca, and L. J. Martinez-Miranda, Submitted Appl. Phys. Lett. manuscript L-0390 (1996).

27P. A. Schultz and E. B. Stechel, To be submitted, Phys. Rev. B (1996).

28N. A. Marks, D. R. McKenzie, B. A. Pailthorpe, M. Bernasconi, and M. Parrinello, Phys. Rev. Lett. 76, 768 (1996).

${ }^{29}$ C. B. Collins, F. Davanloo, D. R. Jander, T. J. Lee, H. Park, and J. H. You, J. Appl. Phys. 69, $7862(1991)$.

30 A. A. Voevodin, M. S. Donley, J. S. Zabinsky, and J. E. Bultman, Surf. Coat. Technol. 77, 534 (1995). 
31W. C. Oliver and G. M. Pharr, J. Mater. Reser. 7 (6), 1564 (1992).

32J. A. Knapp, D. M. Follstaedt, J. C. Barbour, and S. M. Myers, NIMB B127/128, 935 (1997).

33J. A. Knapp, D. M. Follstaedt, J. C. Barbour, S. M. Myers, J. W. Ager III, O. R. Monteiro, and I. G. Brown, Mat. Res. Soc. Symp. Proc. 438, 617 (1997).

${ }^{34}$ C. A. Brookes, in The Properties of Natural and Synthetic Diamond, edited by J. E. Field (Academic, New York, NY, 1992), pp. 515.

35J. B. Pethica, R. Hutchings, and W. C. Oliver, Phil. Mag. A 48, 593 (1983).

36. M. F. Doerner and W. D. Nix, J. Mater. Res. 1, 601 (1986).

37W. C. Oliver and G. M. Pharr, J. Mater. Res. 7, 1564 (1992).

${ }^{38}$ G. M. Pharr and W. C. Oliver, MRS Bull. 17, 28 (1992).

39I. N. Sneddon, Int. J. Engng. Sci. 3, 47 (1965).

40J. W. Harding and I. N. Sneddon, Proc. Phys. Soc. London 78, 169 (1961).

41 J. A. Knapp, D. M. Follstaedt, J. C. Barbour, and S. M. Myers, Nucl. Instr. Meth. B127/128, 935 (1997).

42S. M. Myers, J. A. Knapp, D. M. Follstaedt, and M. T. Dugger, J. App. Phys 83, 1256 (1998).

43J. A. Knapp, D. M. Follstaedt, S. M. Myers, J. C. Barbour, T. A. Friedmann, J. W. Ager III, O.

R. Monteiro, and I. G. Brown, Surf. Coat. Technol., in press .

44J. A. Knapp, D. M. Follstaedt, S. M. Myers, J. C. Barbour, and T. A. Friedmann, J. Appl.

Phys., in press. .

45R. B. King, Int. J. Solids Structures 23, 1657 (1987).

46 ABAQUS ver. 5.7, Hibbitt, Karlsson \& Sorensen, Inc., Pawtucket, RI. .

47 in Mechanical Behavior of Materials, edited by F. A. McClintock and A. S. Argon ((AddisonWesley, Reading, MA, 1966), pp. 276.

${ }^{48} \mathrm{C}$. A. Brookes, in The Properties of Natural and Synthetic Diamond, edited by J. E. Field ((Academic, New York, 1992), pp. 515.

49 J. C. Hay and G. M. Pharr, Mat. Res. Soc. Symp. Proc., in press. . 


\section{Synthesis of cubic Boron Nitride}

\subsection{Thick cubic boron nitride produced by a hybrid ion assisted sputtering technique}

Significant ion irradiation is needed during growth to synthesize cubic boron nitride (cBN) films. This results in large film stresses, which have limited cBN film thicknesses to only a few hundred $\mathrm{nm}$ and represents a significant barrier in the development of $\mathrm{cBN}$ film technology. Using a new hybrid deposition technique, we have synthesized cubic BN films up to $700 \mathrm{~nm}(0.7 \mathrm{~mm})$ thick. A compositional and structural analysis of the films using several standard characterization techniques confirms that relatively thick polycrystalline films with a high $\mathrm{cBN}$ content were synthesized. Thicker $\mathrm{cBN}$ films enable hardness measurements to be undertaken without major substrate effects. Nanoindentation measurements yield hardness values for the cubic BN films up to 60-70 GPa, which are greater than values measured for bulk $\mathrm{cBN}$. The measured elastic modulus was observed to be lower than the bulk, and this can be accounted for by an elastic deformation of the silicon substrate. The mechanical properties of the cubic BN films are discussed with reference to other ultrahard thin films such as diamond and diamondlike carbon. (c) 1997 American Institute of Physics. [SO0218979(97)07116-8]

\subsection{INTRODUCTION}

The cubic phase of boron nitride ( $\mathrm{cBN}$ ) has many desirable properties including a hardness second only to diamond.1,2 a high-temperature oxidation resistance, ${ }^{2,3}$ and the ability to machine ferrous-based metals. ${ }^{2}$ Additionally, the material is a wide-band-gap semiconductor ${ }^{2}$ that can be doped both $\mathrm{p}$ and $\mathrm{n}$ type. ${ }^{4,5}$ Significant ion irradiation during BN film growth is currently necessary to form the $\mathrm{sp}^{3}$-bonded cubic phase over the other, less desirable, $\mathrm{sp}^{2}$-bonded graphitic phases.6,7 Due to the poor crystallinity 8,9 and large compressive film stress 10,11 in the highly irradiated films, hard/protective coating applications are presently more viable than electronic applications. Therefore, accurate mechanical property measurements are important. Several works have reported hardness values of 40-60 GPa using Vickers or Knoop microhardness testing on cBN films. ${ }^{12-15}$ Surprisingly, these high hardness values were obtained for films on soft substrates in which the indent depth was typically a large fraction (50\% or more) of the film thickness. Such excessive indent-depth-to-film-thickness ratios were typically used because of the following constraints: (i) the cBN films were limited to thicknesses of a few hundred nm due to delamination from the ion-induced stress, and (ii)) most microhardness testers have minimum loads of 5-10 g (49-98 mN), which limits the minimum indent depth. Recently, several groups 16,17 have used nanoindentation, which allows for shallower indent depths, to overcome limitation (ii)). However, having sufficiently thick films [limitation (i)] has remained a barrier in the mechanical characterization of cBN films. There is a need for a sufficiently thick film in order to have a reasonable indent depth, which is needed to initiate deformation beyond the elastic regime, as well as to minimize the indentation size effect (discussed in Sec. IV A). This problem is not confined to cBN films. For example, it has been difficult to synthesize high $\mathrm{sp}^{3}$-content amorphous carbon films more than several hundred $\mathrm{nm}$ thick due to large film stresses incurred during growth. 18

Here, we report the synthesis of $\mathrm{cBN}$ films with a high cubic fraction, and up to 700 $\mathrm{nm}(0.7 \mu \mathrm{m})$ thick, using a hybrid deposition technique combining elements from several techniques $16,19-23$ used in the literature. A compositional and structural analysis of the thicker-than-usual $\mathrm{cBN}$ films was performed, and the mechanical properties were evaluated using nanoindentation. We also discuss issues of plastic deformation and mechanical property measurements that are also relevant for other hard ceramic thin-film systems, such as amorphous carbon, diamond, and $\mathrm{CN}_{\mathrm{x}}$. 


\subsection{EXPERIMENTAL PROCEDURE}

\subsubsection{Synthesis}

Cubic BN films were synthesized via a hybrid technique combining elements from several techniques previously used in the literature. The first, and most important element of this technique, is the use of a high-growth-temperature, low-ion-energy process similar to that used by Taylor and co-workers ${ }^{19,24}$ and Kidner et al. ${ }^{20}$ The second element is the use of $\mathrm{B}_{4} \mathrm{C}$ sputter targets as a boron source in cBN syntheses as previously described by Luthje et al. ${ }^{16}$ and Johansson et al. ${ }^{23}$ The third is a technique in which the ion energy is reduced after $\mathrm{CBN}$ nucleation, which has been published by McKenzie et al. ${ }^{21}$ and Hahn et al. 22 In our study, hotpressed $\mathrm{B}_{4} \mathrm{C}$ targets (purity $99.5 \%$ ) were primarily used; however, for comparison, several films were also grown employing pyrolytic graphitic BN targets. A simplified schematic of the deposition system for ion-assisted sputtering is shown in Fig. 31 . This system differs from

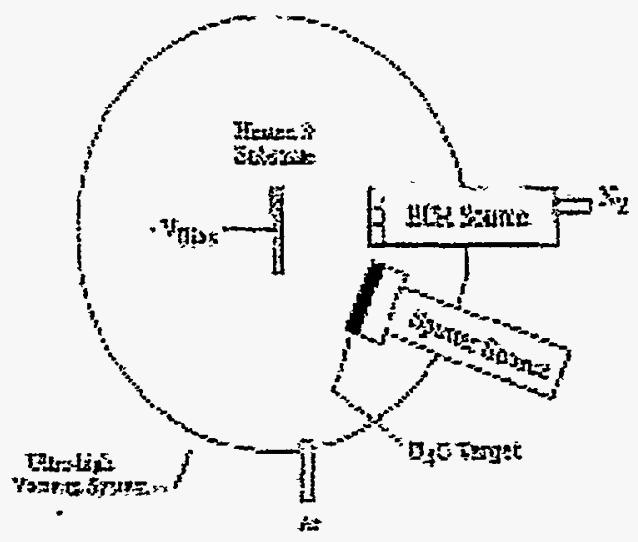
the ion-assisted pulsed laser deposition approach used in our previous studies. $25-28$ The $\mathrm{rf}$ target power on the magnetron sputter source was roughly $50 \mathrm{~W}$ for B4C and $80 \mathrm{~W}$ for BN. 0.5 $\mathrm{mm}$ thick $\mathrm{Si}(100)$ substrates were used. A commercial electron cyclotron resonance (ECR) ion source was directed toward the film/substrate at near-normal incidence, and a negative dc bias of $85-100 \mathrm{~V}$ was applied to the substrate. Generally, the bias was at $-100 \mathrm{~V}$ at the beginning of the run, and was gradually lowered by $\sim 15 \%$ during the first half of the deposition process. The background pressure in the vacuum chamber during sputtering was 3.0-3.5 mTorr, and was primarily argon. The gas fed into the ECR ion source was nitrogen, and hence, most of the ions impinging on the substrate are $\mathrm{N} 2+$ and $\mathrm{N}+$. There are also some incident Ar ions since (i) Ar ions are created in the sputter-source plasma, and (ii) some argon enters the ECR source and is ionized. Net $\mathrm{CBN}$ deposition rates (including sputtering effects) were low and in the range of $15-30 \mathrm{~nm} / \mathrm{h}$. Our previous studies employed ion energies of $>500 \mathrm{eV}$, and ion-flux/deposition-flux ratios of approximately unity to form cBN. The lower ion energies used in this study $(\sim 100 \mathrm{eV})$ required higher ion-flux/deposition-flux ratios for $\mathrm{CBN}$ synthesis, and limitations on the ion flux provided by our ECR source required a lower deposition rate. The deposition temperature was approximately $1000^{\circ} \mathrm{C}$, as measured by a two-color optical pyrometer. On several of the films, a thin ( $<$ a few tens of $\mathrm{nm}$ thick) B4C buffer layer was intentionally deposited on the substrate before BN growth since buffer layers of several non-BN materials have been reported to promote $c B N$ film adhesion. 7,29,30 In this work, no major difference was observed in the short-term adhesion of the films with and without the thin B4C buffer layers; however, a more systematic study is needed before conclusions can be drawn. Film thicknesses were estimated by profilometry on selected samples in which the substrate was masked. On the thickest cBN sample used in this study, some film delamination was observed after long-term ambient exposure, and the thickness was estimated via profilometry between the delaminated and undelaminated regions. The thickness values quoted in this paper are for the total film thickness, i.e., $\mathrm{cBN}$ plus the very 
thin interlayers [turbostratic BN (discussed in Sec. III) and/or B4C], and hence, the actual $\mathrm{cBN}$ portion will be a few tens of $\mathrm{nm}$ less than the cited value.

\subsubsection{Characterization}

Phase identification was provided by Fourier transform infrared spectroscopy (FTIR) and transmission electron microscopy(TEM). Compositional analysis was performed using sputter-Auger electron spectroscopy and Rutherford backscattering spectrometry (RBS)/elastic recoil detection(ERD). Surface morphology was examined by scanning electron microscopy, and microstructural analysis was provided by cross-sectional transmission electron microscopy.

The primary method for phase identification in the BN films was FTIR spectroscopy. For $\mathrm{sp}^{2}$-bonded $\mathrm{BN}$, such as crystalline hexagonal $\mathrm{BN}(\mathrm{hBN})$ or its poorly crystalline variant turbostratic $B N(t B N)$, there are two characteristic peaks at approximately 1370 and $800 \mathrm{~cm}^{-1}$. $\mathrm{Sp}^{3}$-bonded cubic BN has a characteristic peak at approximately $1065 \mathrm{~cm}^{-1}$. The IR spectra were obtained in the reflectance mode at approximately normal incidence, and were ratioed to the spectrum of a clean silicon wafer. TEM specimens, prepared by a standard procedure of mechanical grinding and ion milling described elsewhere, ${ }^{9}$ were observed at $120 \mathrm{kV}$ in a JEOL $1200 \mathrm{EX}$ electron microscope, and at $400 \mathrm{keV}$ in a JEOL $4000 \mathrm{EX}$ high-resolution (HR) microscope. Scanning electron microscopy was performed at a beam voltage of $10 \mathrm{kV}$. Sputter-Auger electron spectroscopy was used to measure the film purity, and was performed at an electron-beam voltage of $5 \mathrm{kV}$. Rutherford backscattering spectrometry and elastic recoil detection were used to determine film stoichiometry and purity, by a procedure similar to that outlined previously. 28

Nanoindentation measurements were conducted on a Nanoindenter II equipped with a Berkovitch indenter tip. The tip was calibrated using the common method of indenting fused silica at several displacement values, and extracting the tip-area-function coefficients by fitting the contact data to an eighth-order polynomial. 31,32 The loading rate was $100 \mu \mathrm{N} / \mathrm{s}$ and the unloading rate was $75 \mu \mathrm{N} / \mathrm{s}$. A 15-30 s hold segment was used after loading, and a $30 \mathrm{~s}$ hold segment was used after unloading to $20 \%$ of the maximum load. This enabled the

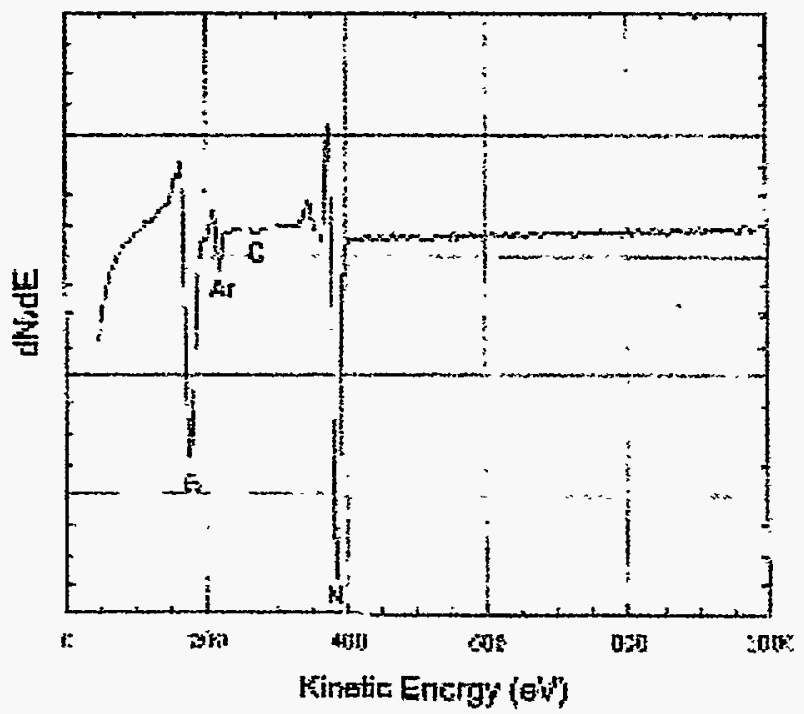

Fig. 3-2. Auger electron spectroscopy on a typical cBN film deposited from a B4C target. The spectra was taken after Ar+ ion sputtering of the surface. thermal drift to be measured, which was used to correct the measured values. Hardness and elastic modulus values were obtained by analyzing the unloading curve using the standard Oliver-Pharr method. 32 The indent depth was $100 \mathrm{~nm}$. For a particular hardness or modulus value, the error is \pm the standard deviation of repeated measurements, unless noted otherwise.

Modeling of the nanoindentation loading/unloading curves was performed using an ABAQUS finite element code/procedure described elsewhere. 33 Input parameters include the elastic modulus of the substrate, the thickness of the layers, the areafunction coefficients of the indenter tip, and the approximate stress in the film prior to the indentation process. We took the stress to be $3 \mathrm{GPa}$ and compressive, the approximate value 
measured in our previous cBN films. ${ }^{11}$

\subsection{COMPOSITIONAL AND MICROSTRUCTURAL ANALYSIS}

Figure 3-2 shows a typical Auger electron spectrum of a cBN film deposited from a $\mathrm{B} 4 \mathrm{C}$ target. The argon observed in the spectra is partly an artifact of the argon ions used to sputter clean the film. Using published sensitivity factors, ${ }^{34}$ the carbon content was estimated to be less than $3 \%$. Rutherford backscattering spectrometry measurements, using enhanced cross sections for carbon that are 5.6 times larger than the Rutherford cross sections, showed no detectable carbon above the background. The counting statistics give an uncertainty to this measurement of $2 \%$ as a result of the background signal from the $\mathrm{Si}$ substrate. The absence of carbon was also observed in the ERD spectra. Previous studies 16,23,35 employing B4C targets for $\mathrm{cBN}$ film synthesis have found that the carbon content of the films can be as low as $\approx 5 \%$. The technique employed here, which uses a higher fraction of nitrogen in the ion beam and higher deposition temperatures, yields $\mathrm{cBN}$ films with an even lower carbon content. RBS measurements yield a $B / N$ ratio of 1.0 , confirming that the films are stoichiometric, and ERD measurements show less than $0.5 \%$ hydrogen.

Figure 3-3(a) shows the FTIR spectra for a BN film in which the ratio of ion flux (J) to deposition flux (a), $\mathrm{J} /$ a, was intentionally varied across the sample. The ion flux was a maximum at the center of the sample (labeled C). This is the thinnest region of the film because most of the depositing atoms were sputtered away during film growth. The conditions under which this occurs have been commonly referred to in the $\mathrm{cBN}$ literature as the "resputtering region." In the thinnest area, region $\mathrm{C}$, the $\mathrm{cBN}$ peak occurs at about $1077 \mathrm{~cm}^{-1}$, and the IR reflectance is $\approx 150 \%$, a larger peak amplitude than we have reported previously. 27,28 The peak at $\approx 1390 \mathrm{~cm}^{-1}$ is associated with $\mathrm{sp}^{2}$-bonded BN. As $\mathrm{J} / \mathrm{a}$ is reduced, the film thickness and the amplitude of the cBN IR peak increase significantly,
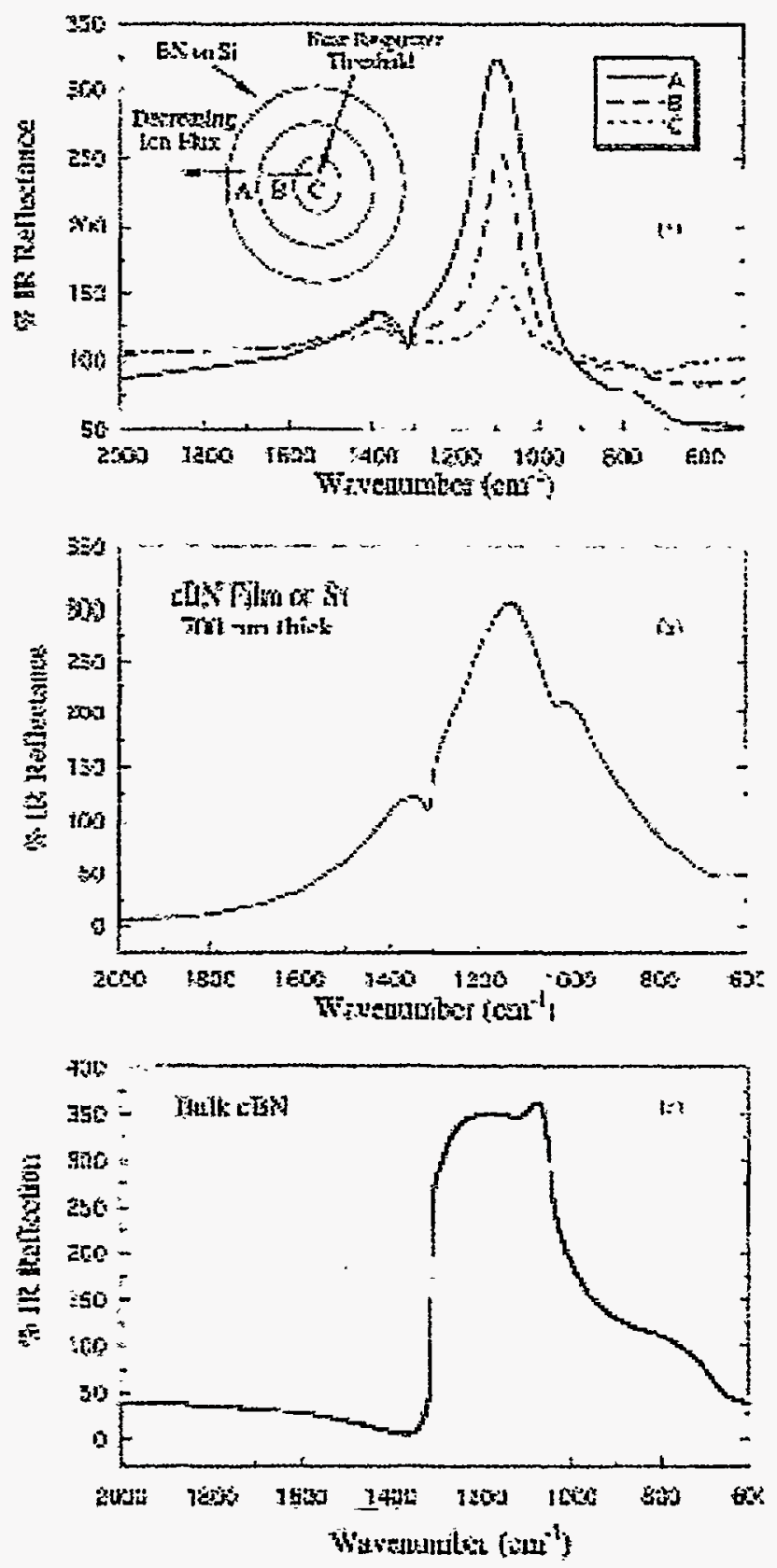

Fig. 3-3 Fourier transform infrared spectra of (a) a BN film in which the ratio of ion flux to deposition flux was varied across the sample, (b) an approximately $0.7 \mu \mathrm{m}$ thick $\mathrm{BN}$ film, and (c) bulk polycrystalline $\mathrm{cBN}$. 
with a much smaller increase in the $\mathrm{sp}^{2}$-bonded BN IR peak. The $\mathrm{cBN}$ peak broadens and shifts to higher wave numbers, reaching $\approx 1095 \mathrm{~cm}^{-1}$ in area A. Using the conventional method of measuring relative IR peak amplitudes, 7,28 we estimate $>85 \%$ $\mathrm{cBN}$ in the films. Peak broadening and a shift to higher wave numbers is the expected behavior for the IR reflectivity of $\mathrm{cBN}$ with increased film thickness. 28,36 Area $A$ is approximately $0.5 \mu \mathrm{m}$ thick. Beyond this thickness, the IR reflectance saturates at a little above $300 \%$, and the peak tends to widen and take on more bulklike features. This is shown in Fig. $3(\mathrm{~b})$ for $\mathrm{a}=0.7 \mu \mathrm{m}$ thick, predominantly $\mathrm{cBN}$ film. Figure 3 (c) shows the FTIR reflectance spectrum for bulk, polycrystalline $\mathrm{cBN}, 37$ which

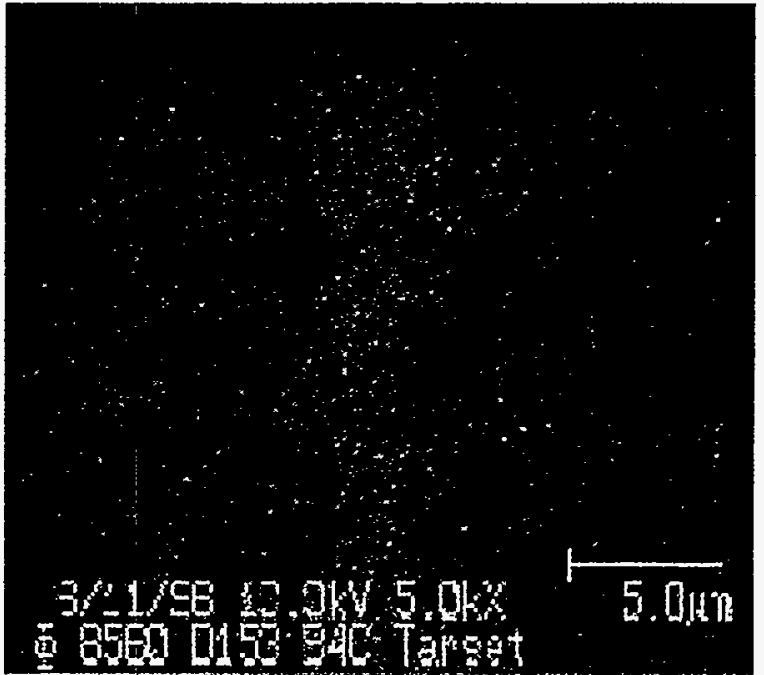

Fig. 3-4 Scanning electron micrograph of $a \approx 0.5$ $\mu \mathrm{m}$ thick cBN film. looks significantly different than the thin-film spectra shown in Fig. 3(a). Also, no significant differences were detected in the IR spectra of thin films synthesized via B4C or BN targets.

Figure 3-4 shows a typical scanning electron micrograph of $a=0.5 \mu \mathrm{m}$ thick $\mathrm{cBN}$ film. The film is smooth on this scale (magnification $\approx 5000 \mathrm{X}$ ), more like $\mathrm{sp}^{3}$-bonded amorphous carbon films than typical diamond films. 38

Many reports in the literature $8,9,39,40$ have shown that cBN films generally form in a layered manner that commences with growth of a graphitic (turbostratic) BN layer and is followed by growth of the cubic phase. The distribution of the graphitic and cubic phases in
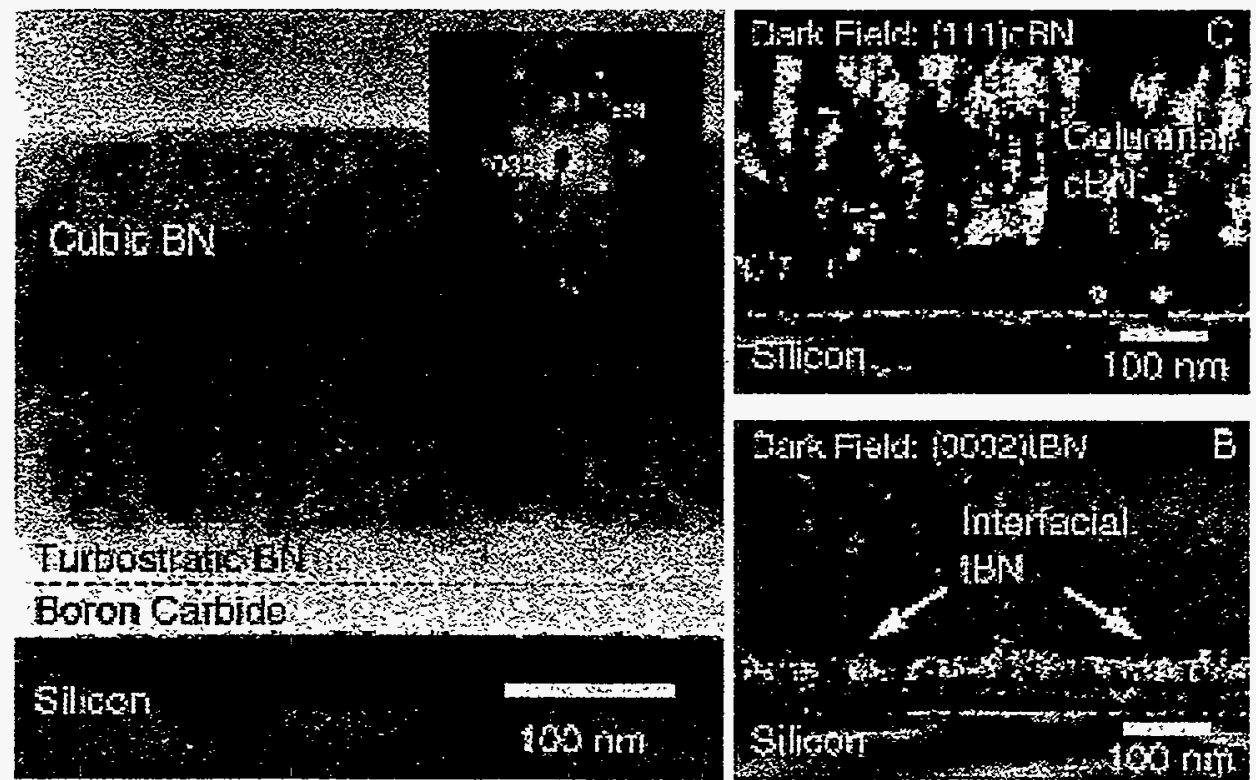

Fig. 3-5. (a) Bright-field TEM image of a cBN film with diffraction inset, (b) dark-field TEM image obtained from the tBN (0002) diffraction ring, and (c) dark-field TEM image obtained from the $\mathrm{CBN}(111)$ diffraction ring. 
films produced by the current method was investigated using several transmission electron microscopic techniques. Figures 3-5(a),3-5(b), and 3-5(c) show a series of bright- and dark-field TEM micro-graphs of a cBN film in cross section. Figure $3-5(b)$ is a dark-field micrograph formed by positioning a small objective aperture over a section of the $t B N(0002)$ diffraction ring; Fig.3-5(c) was formed similarly, but in this case a section of the cBN $\{111\}$ ring was used. Thus, the bright $\sim 40 \mathrm{~nm}$ wide strip of contrast near the interface in Fig. 3-5(b) corresponds to a layer of graphitic boron nitride. (Note that this layer does not start immediately at the silicon interface due to the intentional deposition of an initial $40 \mathrm{~nm}$ thick layer of sputtered B4C.) The bright regions in Fig. 3-5(c) correspond to columnar cBN grains, which form only above the layer of graphitic $B N$.

A bright-field TEM image of a thicker $\mathrm{CBN}$ film in crosssection is shown in Fig. 3-6. There is a distinct change in contrast in the microstructure showing a layer of brighter material near the interface. Selected area electron diffraction (inset) confirms that the majority of the film is in the cubic form. The $\mathrm{cBN}\{111\},\{220\}$, and $\{311\}$ rings are labeled. However, the presence of the intensity from the tBN (0002) diffraction ring indicates that some graphitic boron nitride remains.

Figure 3-7 shows a HRTEM image taken near the top of the same film examined in Fig. 3-5. The lattice fringe contrast is consistent with the $2.09 \AA$ spacing of the $\mathrm{cBN}\{111\}$ planes. Analysis of the selected area diffraction patterns indicates that both phase have a preferential crystallographic texture (tBN: [0002] inplane orientation; cBN: [111] in-plane orientation) similar to previous observations for $\mathrm{tBN}$ (Refs. 30 and ${ }^{31}$ ) and cBN. ${ }^{32}$ In summary, the microstructural characterization confirms that the $\mathrm{cBN}$ films produced by the new hybrid process are relatively thick, and that $\mathrm{cBN}$ growth is still preceded by formation of a layer of $\mathrm{sp}^{2}$-bonded graphitic $\mathrm{BN}$.

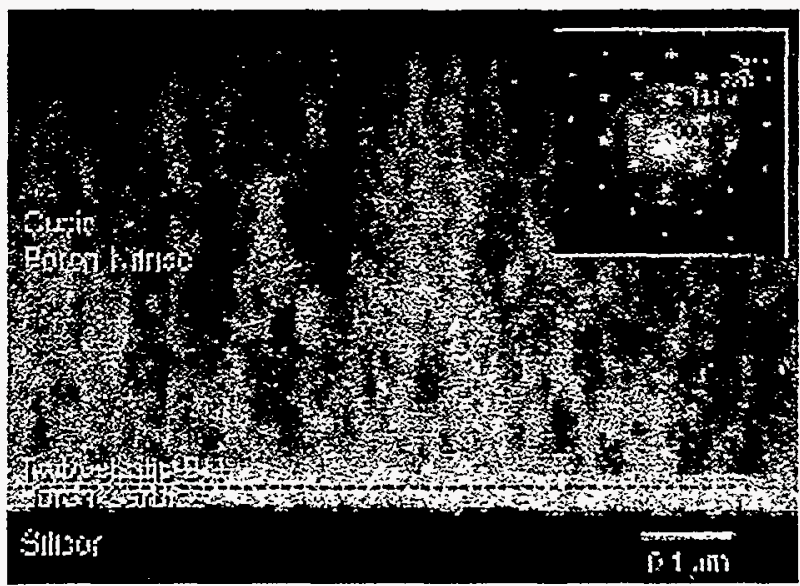

FIG. 3-6. Bright-field TEM image of $\mathrm{a} \approx 0.5 \mu \mathrm{m}$ thick $\mathrm{cBN}$ film in cross section, with a diffraction inset indicates that both phases have a preferential crystallographic texture (tBN: [0002] in-plane orientation; cBN: [111] in-plane orientation) similar to previous observations for tBN (Refs. 8 and 40 ) and cBN.41

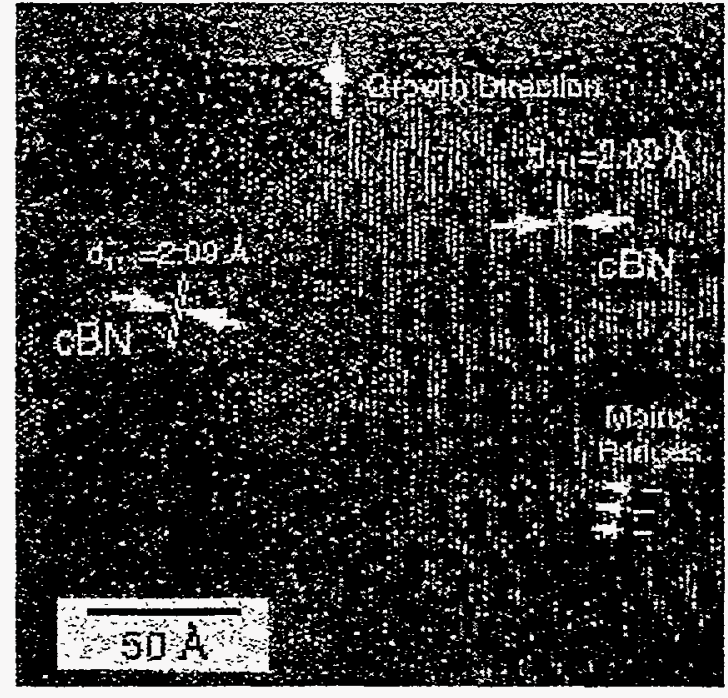

Fig. 3-7. High-resolution TEM image of the cBN film shown in Fig. 5. 


\section{MECHANICAL PROPERTIES OF CBN FILMS}

\subsubsection{Measuring the mechanical properties of hard ceramics by nanoindentation}

Determining the mechanical properties of bulk materials from indentation methods is, in general, nontrivial. For example, in the indentation of most metals, the deformation is almost entirely plastic, while for many ceramics the deformation has a much larger elastic component. Figure 3-8 shows nanoindentation loading/unloading curves, and illustrates the difference in unloading behavior between highly plastic and highly elastic responses. In the highly plastic case, typical of most metals, the displacement at maximum load is close to the displacement after the load is removed, i.e., a large amount of permanent deformation has occurred. For the other case, which is typical of many ceramics, the indenter displacement after the load is removed is considerably smaller than the maximum displacement, due primarily to an elastic recovery of the indented material. Large amounts of elastic recovery can lead to measurement errors in micro-hardness testing; for the technique of nanoindentation, where the elastic recovery can, in principle, be accounted for, a large elastic recovery makes the analysis process more problematic.

In measuring the mechanical properties of films on substrates, further complications arise. Generally, for a film on a substrate, as the indenter is displaced into the material, the film will first elastically deform, then plastically deform. With further indenter displacement, the substrate will elastically deform, and then plastically deform. Thus, for a thin film, part of the observed elastic recovery can be due to the substrate pushing back out elastically after the load is removed. In performing nanoindentation on thin films, there are two competing factors one must consider: (i) the smaller the indenter displacement, the less one has to be concerned about substrate effects, and (ii) the larger the indenter displacement, the less one has to be concerned about the indentation size effect 42 (ISE), for which the hardness can increase with the decreasing load for very small displacements. There are different explanations for this effect, ${ }^{42}$ including strain-rate effects. ${ }^{43}$ The indentation size effect has generally been observed in microhardness measurements on hard ceramic materials, including bulk $\mathrm{cBN}$ and diamond. ${ }^{44}$ The ISE effect is expected to be more pronounced in microhardness measurements, than in nanoindentation, since in nanoindentation elastic recovery is at least in part accounted for. For very hard materials, larger displacements are also desirable so that the deformation is taken beyond the elastic regime. We have chosen an indenter displacement of $100 \mathrm{~nm}$ as a compromise.

Bull et al. ${ }^{45}$ have proposed a model for the indentation size effect in ceramics that provides insight into why the observed elastic recovery might be much higher for a ceramic than a metal. In the indentation of a ceramic, the deformation under the indenter is said to occur in discrete slip bands. This is in contrast to a metal, where a more continuous, homogeneous plastic zone forms ahead of the tip. During the unloading segment, recovery of 
the elastic component of each of the slip bands reduces the size of the impression, resulting in an apparent increase in the hardness. For hard ceramic films on softer substrates, it has been suggested 46 the film/substrate can flex/bend upon loading, and it can bend back during unloading, which would increase the observed elastic recovery. Note that for this to occur, the films must be so hard/strong that it takes less load to bend the film/substrate over a macroscopic area than it does to induce plastic/elastic deformation in a microscopic area.

\subsubsection{Results}

The mechanical property results are summarized in Table I. The cubic BN films appear to be very hard, with hardnesses as high as $60-70$ $\mathrm{GPa}$, exceeding our measured bulk value of 50-55 GPa. A typical loading/unloading (L/UL) curve from the thickest cBN film tested is shown in Fig.39(a). The measured elastic modulus is somewhat lower than the expected bulk values, which will be discussed in the next section. In Fig. 3-9(b), the film L/UL curve for a bulk cBN sample is shown. The elastic recovery for bulk $\mathrm{cBN}$ was measured to be $\sim 65 \%$, which is not unusual for a hard ceramic at these indentation depths. For example, we measured an elastic recovery of $60 \%$ for $\mathrm{Si}(100)$ at the same indent depth. The cBN films generally had elastic recoveries $>80 \%$. Some of the difference in elastic recovery (between the film and the bulk) may be due to substrate effects, as discussed below.

TABLE I. Mechanical properties of cBN films and selected references.

\begin{tabular}{ccccccc}
\hline \hline Sample & $\begin{array}{c}\text { Sputter target } \\
\text { used }\end{array}$ & $\begin{array}{c}\approx \text { Film } \\
\text { thickness } \\
\text { (nm) }\end{array}$ & $\begin{array}{c}\text { Indent } \\
\text { depth } \\
\text { (nm) }\end{array}$ & $\begin{array}{c}\text { Mean } \\
\text { (GPa) }\end{array}$ & $\begin{array}{c}\text { Mean } \\
\mathrm{E} \\
(\mathrm{GPa})\end{array}$ & $\begin{array}{c}\approx \% \\
\text { Recovery }\end{array}$ \\
\hline cBN film & $\mathrm{B}_{4} \mathrm{C}$ & 700 & 100 & $68 \pm 11$. & $530 \pm 60$ & $80 \%-$ \\
cBN film & $\mathrm{B}_{4} \mathrm{C}$ & 700 & 135 & $47 \pm 13$ & $4200 \pm 50$ & $80 \%$ \\
cBN film & $\mathrm{B}_{4} \mathrm{C}$ & $550-$ & 100 & $69 \pm 6$ & $360 \pm 20$ & $85 \%$ \\
cBN film & $\mathrm{B}_{4} \mathrm{C}$ & $550-$ & 135 & $59 \pm 5$ & $305 \pm 15$ & $80 \%$ \\
cBN film & $\mathrm{B}_{4} \mathrm{C}$ & 400 & 100 & $55 \pm 8$ & $320 \pm 20$ & $80 \%$ \\
cBN film & $\mathrm{BN}$ & 450 & 100 & $58 \pm 14$ & $330 \pm 25$ & $80 \%$ \\
cBN film & $\mathrm{BN}$ & $450-$ & 100 & $55 \pm 29$ & $290 \pm 55$ & $80 \%$ \\
tBN film & $\mathrm{B}_{4} \mathrm{C}$ & $700+$ & 100 & $2 \pm 0.5$ & $50 \pm 10$ & $\mathrm{c}$ \\
Bulk cBN $^{\mathrm{a}}$ & N/A & Bulk & 100 & $53 \pm 14$ & $850 \pm 150$ & $65 \%$ \\
$\beta$ SiC film & N/A & 1000 & 100 & $37 \pm 5$ & $370 \pm 35$ & $55 \%$ \\
Silicon & N/A & Bulk & 100 & $13 \pm 1$ & $190 \pm 5$ & $60 \%$ \\
\hline \hline
\end{tabular}

a \%recovery as defined by Page and Hainsworth, ${ }^{50}$ i.e., [ =(depth at maximum load- depth after load removed)/depth at maximum load].

'Deposited under the same conditions as the cBN films except at a lower ion flux.

c Not calculated since significant creep was observed in the L/UL curve.

${ }^{d}$ Bulk, polycrystalline, and translucent $\mathrm{cBN}$, manufactured by Sumitomo, Inc.

${ }^{\circ}$ Cubic SiC films on Si substrates available commercially from CREE, Inc. 


\subsection{Analysis and discussion}

\subsubsection{Why the depressed elastic modulus for the films?}

The measured nanoindentation elastic modulus ${ }^{47}$ of bulk $\mathrm{cBN}$ is consistent with literature values (of 800-900 GPa for the Young's modulus). $11,48,49$ Since nanoindentation has not been previously reported, to the best of our knowledge, on bulk cBN prior to this study, we use literature values of the Young's modulus for comparison. This is reasonable, since the Voight/Reuss-averaged Young's modulus has been equated to the nanoindentation modulus, particularly for elastically isotropic materials. 32

The measured elastic modulus of the $\mathrm{cBN}$ films is lower than the measured value for bulk cBN. Differences in grain size and small differences in density are not expected to affect the elastic modulus greatly, and most of the low modulus $\mathrm{sp}^{2}$-bonded $\mathrm{BN}$ is believed to be at the substrate/film interface. From the data shown in Table I, as well as additional data not shown, a higher elastic modulus is obtained from the thicker films, and for a given film, the elastic modulus decreases with increasing indenter penetration. This suggests that the depressed elastic modulus is due primarily to substrate effects. We use the term "substrate effects" in this work to include the effect of any non-cBN interlayers as well as the $\mathrm{Si}$ substrate.

Hainsworth and Page ${ }^{50}$ recently proposed a method for estimating substrate effects in nanoindentation by examining the loading portion of the loading/unloading curve. The displacement value where the load versus the displacement-squared curve deviates from linearity is said to denote the onset of the substrate effects, beginning with an elastic deformation of the substrate. To test this new method, we indented to a $100 \mathrm{~nm}$ depth on bulk $\mathrm{Si}$, and a $1000 \mathrm{~nm}$ thick $\beta-\mathrm{SiC}$ film on Si. The load versus the displacement-squared curve did not deviate from linearity, as expected. In Fig. 3-10, we plot the load versus the displacementsquared curve for $\mathrm{cBN}$ films that are approximately 700 and $450 \mathrm{~nm}$ thick. As expected, the curve for the $450 \mathrm{~nm}$ thick film shows a significant deviation from linearity at a smaller indenter displacement value than the 700 $\mathrm{nm}$ film. Note that in both instances, substrate effects begin to play a role at approximately the same ratio of indent depth to film thickness of ' 0.12 (i.e., at an indent depth of $12 \%$ of the film thickness). Additionally, we have performed finite-element modeling of the loading/unloading curve from the $700 \mathrm{~nm}$ thick film (see Fig. 3-11). The modeling code takes into account the Si substrate and the $t$ BN interlayer, i.e., it accounts for "substrate effects," and yields an elastic modulus for the film of $730 \mathrm{GPa}$, which is much closer to the bulk value of $800 \mathrm{GPa}$. Modeling of the L/UL curve from the bulk sample yielded $800 \mathrm{GPa}$, which is a reasonable value.

There are instances in the literature where lower-than-expected elastic moduli were observed for other types of hard films, such as diamond and diamondlike carbon. For example, Savvides and Bell 50 used

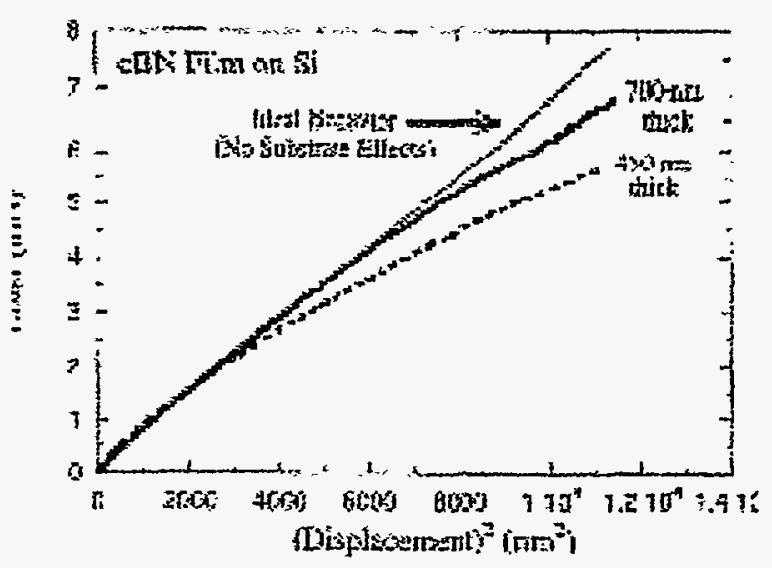

Fig. 3-10. The load vs. (displacement), obtained from the loading portion of the nanoindentation curves, for $\mathrm{cBN}$ films of different thicknesses. According to the Hainsworth-Page model, the displacement value where the curves deviate from linearity indicates the onset of substrate effects. 
nanoindentation to measure the mechanical properties of $3 \mathrm{~mm}$ thick diamond films, and the measured hardness was at least as large as that for bulk diamond; however, the measured nanoindentation elastic modulus of the films was lower than expected. They speculated that the depressed elastic modulus arose from a small amount of hydrogen ( 1 at. \%) in the films, which would form lower spring constant C-H bonds. We offer another possibility for their findings, namely substrate effects. From the loading/unloading curves shown in their paper, the sample indented to the shallower thickness shows a higher elastic modulus, consistent with this explanation.

\subsubsection{Plastic deformation of $\mathrm{cBN}$, and ultrahard films in general \\ Nanoindentation indicates that several} of the cBN films are harder than bulk polycrystalline $c B N$. It is not uncommon for the hardness of ceramic films to be slightly higher than their bulk counterparts. ${ }^{51}$ This could be due to factors such as a larger grain size for the bulk material or the fact that the bulk material may not have been sintered to its full theoretical density. 52 The indentation size effect is not expected to contribute to the difference in measured hardnesses since the films and bulk material were indented to the same depth. The loading/unloading curves for the $\mathrm{cBN}$ films [see Fig. 3-9(a)] indicate a significant amount of elastic recovery with a small amount of residual, permanent deformation. There is generally no evidence of cracking in the loading/unloading curves; however, a small amount of microcracking might not be observable using this technique.

As noted above, finite-element modeling of the L/UL curves for bulk $\mathrm{cBN}$ and $\mathrm{cBN}$ films yielded reasonable elastic modulus values. Modeling of the plastic behavior for these materials is not as straightforward, however. The yield stress values generated by finiteelement modeling of a cBN film and bulk $\mathrm{cBN}$ were 51 and $37 \mathrm{GPa}$, respectively. For metals, Tabor ${ }^{53}$ observed that the hardness is equal to approximately three times the yield stress. For hard ceramic materials, a different Tabor-like relation has been suggested, where the hardness is approximately 1.5-1.8 times the yield stress. ${ }^{54-56}$ Thus, the hardness values estimated from the finite-element modeling results are 55-66 and 76-92 GPa, for bulk cBN and cBN films, respectively. These hardness values are a little high, but reasonable. We note that the same finite-element code applied to the L/UL curves of diamond-like amorphous carbon films with a high $\mathrm{sp}^{3}$ content gave similar values for the yield stress. ${ }^{33}$

The loading/unloading curves of the cBN films [see Fig.3-9(a)] show a small amount of residual, permanent deformation along with a significant amount of elastic recovery. How this plastic deformation occurs in $\mathrm{cBN}$ films and other hard materials is an interesting issue. In general, plastic deformation in crystalline materials occurs through the motion of dislocations. Dislocation movement, although possible, 57,58 should be very limited in materials such as $\mathrm{cBN}$ and diamond 44,58 at ambient temperature (i.e., the temperature at which the indentation measurements were performed). Gilman 59,60 has proposed that dislocation mobility in hard ceramic materials, such as silicon and diamond, is limited because of large energy gradients at dislocation kinks. Note that this mechanism would not be able to account for the large hardness observed in amorphous $\mathrm{sp}^{3}$-bonded carbon 55 since this material does not have longrange order, and traditional dislocation theory is not applicable. 


\subsubsection{Future work}

As noted in the introduction, hard-coating applications are presently more viable than microelectronic applications for $\mathrm{cBN}$ films. Mechanical-property measurements of the films are, therefore, important, particularly since it cannot be taken for granted that films with a high $c B N$ content are necessarily hard. 61 The deposition process used here enables relatively thick $\mathrm{cBN}$ films to be synthesized. However, the relatively high temperatures $\left(1000^{\circ} \mathrm{C}\right)$ employed in the current process are not well suited for the general commercialization of $\mathrm{cBN}$ film technology. Reduction of the deposition temperature will require an optimization of other factors to reduce residual film stress and promote adhesion. Moving to lower ion energies, as well as maximizing the reduction in ion energy that can be tolerated after $\mathrm{cBN}$ nucleation, are possible ways of reducing the residual stress even further. Very high-ion-flux sources will be needed to use lower ion energies at practical deposition rates.

It has been reported in the literature than non-cBN buffer layers can enhance $\mathrm{cBN}$ film adhesion. Optimizing this effect may also be useful, particularly if the buffer layers inhibit water diffusion to the film/substrate interface, which has been suggested to contribute to the delamination of cBN films under ambient conditions. ${ }^{62}$ An additional possibility is to alternate $\mathrm{CBN}$ with thin layers of a hard, lower-stress material (such as B $4 \mathrm{C}$ or TiN) in a multilayer structure. We have observed that the adhesion can be improved in such a structure without a significant change in the net $\mathrm{cBN}$ content. ${ }^{63}$ If the material is reasonably hard, and has a lower shear elastic constant than $\mathrm{CBN}$, the multilayer could have a hardness even higher than that of $\mathrm{CBN}$ based on our current understanding of multilayer hardening. 64,65

\subsection{SUMMARY}

By combining several deposition techniques previously used in the literature, we have synthesized cubic BN films up to $700 \mathrm{~nm}(0.7 \mathrm{~mm})$ thick. A compositional and structural analysis of the films using several standard characterization techniques confirms that thick BN films with a high $\mathrm{cBN}$ content were synthesized. The thicker cubic BN films enabled hardness measurements to be undertaken without major substrate effects. Encouragingly, nanoindentation measurements yield hardness values for the cubic BN films up to 60-70 GPa, which are greater than values measured for bulk $\mathrm{cBN}$. The nanoindentation elastic modulus (determined by the Oliver-Pharr method) was found to be lower than the expected bulk value, due to the elastic deformation of the silicon substrate. Finite-element modeling of the $\mathrm{cBN}$ films yields a reasonable value for the elastic modulus, and confirms that the films are very hard. Measurement of the mechanical properties of cubic BN films, and ultrahard films in general, was also discussed.

\subsection{ACKNOWLEDGMENTS}

The authors wish to thank Greg Cardinale and Neville Moody for useful discussions.

\subsection{References}

${ }_{1}^{1}$ H. Holleck, J. Vac. Sci. Technol. A 4, 2661 (1986).

2L. Vel, G. Demazeau, and J. Etourneau, Mater. Sci. Eng. B 10, 149 (1991).

3R. C. DeVries, GE CRD Report No. 72 CRD178 (1972).

${ }_{5}^{4}$. H. Wentorf, Jr., J. Chem. Phys. 36, 1990 (1962).

50 . Mishima, in Synthesis and Properties of Boron Nitride, edited by J. J.Pouch and S. A. Alterovitz (Brookfield: Trans. Tech., 1990), Vol. 54-55, pp. 313.

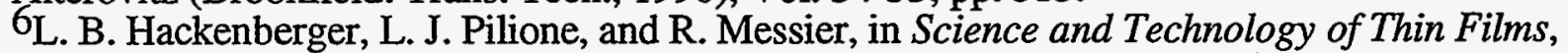
edited by F. Matacotta and G. Ottaviani (World Scientific, Singapore, 1996). 
7P. B. Mirkarimi, K. F. McCarty, and D. L. Medlin, Mater. Sci. Rep.(to be published) .

8D. J. Kester, K. S. Ailey, R. F. Davis et al., J. Mater. Res. 8, 1213 (1993).

9D. L. Medlin, T. A. Friedmann, P. B. Mirkarimi et al., J. Appl. Phys. 76, 295 (1994).

10D. R. McKenzie, W. D. McFall, W. G. Sainty et al., Diam. Relat. Mater. 2, 970 (1993).

11 G. F. Cardinale, D. G. Howitt, K. F. McCarty et al., Diam. Relat. Mater. 5, 1295 (1996).

${ }^{12}$ Y. Andoh, S. Nishiyama, H. Kirimura et al., Nucl. Instrum. Methods Phys. Res. B 59/60, 276 (1991).

13 Nishiyama, N. Kuratani, A. Ebe et al., Nucl. Instrum. MethodsPhys. Res. B 80/81, 1485 (1993).

14W. L. Lin, Z. Xia, Y.L. Liu et al., Mater. Sci. Eng. B 7, 107 (1990).

15T. Wada and N. Yamashita, J. Vac. Sci. Technol. A 10, 515 (1992).

16H. Luthje, K. Bewilogua, S. Daaud et al., Thin Solid Films 257, 40 (1995).

17 M. P. Johansson, H. Sjostrom, and L. Hultman, J. Mater. Res. (submitted) .

$18 \mathrm{M}$. Chhowalla, Y. Yin, G. A. J. Amaratunga et al. " "Highly tetrahedral amorphous carbon

films with low stress," Appl. Phys. Lett 69 (16), 2344 (1996).

${ }^{19}$ C. A. Taylor II, R. Clarke, and S. Fahy, (unpublished) .

20S. Kidner, C. A. Taylor II, and R. Clarke, Appl. Phys. Lett. 64, 1859 (1994).

${ }^{21}$ D. R. McKenzie, W. D. McFall, H. Smith et al., Nucl. Instrum. Methods Phys.Res. B 106, 90 (1995).

22 J. Hahn, F. Richter, R. Pintaske et al., Surf. Coat. Technol. (to be published). .

23 M. P. Johansson, I. Ivanoz, L. Hultman et al., J.Vac. Sci. Technol. A 14, 3100 (1996).

24C. A. Taylor II and R. Clarke, Appl. Phys. Lett. (to be published) .

25P. B. Mirkarimi, K. F. McCarty, G. F. Cardinale et al., J. Vac. Sci. Technol. A 14, 251 (1996).

26P. B. Mirkarimi, D. L. Medlin, K. F. McCarty et al., Appl.Phys. Lett. 66 (1995).

27P. B. Mirkarimi, K. F. McCarty, D. L. Medlin et al., J. Mater. Res. 9, 2925 (1994).

28 T. A. Friedmann, P. B. Mirkarimi, D. L. Medlin et al., "Ion-assisted pulsed laser deposition of cubic boron nitride films," J. Appl. Phys. 76 (5), 3088 (1994).

29 M. Okamato, H. Yokoyama, and Y. Osaka, Jpn. J. Appl. Phys. 29, 930 (1990).

30T. Ikeda, Y. Kawate, and Y. Hirai, J. Vac. Sci. Technol. A 8, 3168 (1990).

${ }^{31}$ Nanoinstruments, Nanoindenter II Operating Instructions, Version 2.0.Nanoinstruments, 1994. .

32W. C. Oliver and G. M. Pharr, "An improved technique for determining hardness and elastic-modulus using load and displacement sensing indentation experiments," J. Mater. Reser. 7 (6), 1564 (1992).

33J. A. Knapp, D. M. Follstaedt, J. C. Barbour et al., in Materials Modification and Synthesis by Ion Beam Processing, edited by D. Alexander, N. Cheung, B. Park et al.

(MaterialsResearch Society, Pittsburgh, 1996), Vol. 438.

34 Handbook of Auger Electron Spectroscopy (Physical Electronics, EdenPrairie, 1976) .

35 A. Schutze, K. Bewilogua, H. Luthje et al., Surf.Coat. Technol. 74/75, 717 (1995).

36Note that it has been suggested that factors such as film stress, and crystallite size effects

during nucleation, can also shift the IR peak in cBN films. .

37 Bulk polycrystalline cBN manufactured by Sumitomo, Inc .

38Z. Feng, S. Anders, A. Anders et al., Diam. Relat. Mater. 5, 1080 (1996).

$39 \mathrm{~K}$. Inagawa, K. Watanabe, K. Saitoh et al., Surf. Coat.Technol. 39/40, 253 (1989).

40D. R. McKenzie, D. J. H. Cockayne, D. A. Muller et al., J. Appl. Phys. 70, 3007 (1991).

41D. L. Medlin, T. A. Friedmann, P. B. Mirkarimi et al., J. Appl. Phys. 79, 3567 (1996).

42P. M. Sargent, in Microindentation Techniques in Science and Engineering, edited by P.

Blau and B. Lawn (ASTM, Ann Arbor, 1985), pp. 160.

43 M. F. Doerner and W. D. Nix, J. Mater. Res. 1, 601 (1986).

${ }^{44}$ C. A. Brookes, in The Properties of Natural and Synthetic Diamond, edited by J. E. Field

(Academic, New York, NY, 1992), pp. 515-546.

${ }^{45}$ S. J. Bull, T. F. Page, and E. H. Yoffe, Philos. Mag. Lett. 59, 281 (1989). 
46M. R. McGurk, H. W. Chandler, P. C. Twigg et al., Surf. Coat. Technol. 68/69, 576 (1994). 47 The nanoindentation modulus is obtained by a common procedure outlined in Refs. 31 and 32 (where a Poisson's ratio for the film is assumed; we used 0.25). As noted by Hubbard et al. (J. Appl. Phys. 72, 4466(1992)), the nanoindenter measures an (unknown) average modulus, nominally weighted towards Young's modulus along the direction of the film normal. .

${ }^{48}$ S. V. Hainsworth and T. F. Page, in Thin Films: Stresses and Mechanical Properties VI, edited by W. W. Gerberich, H. Gao, J.E. Sundgren et al. (Materials Research Society, Pittsburgh, 1996), Vol. 436.

${ }^{49}$ N. Savvides and T. J. Bell, "Hardness and elastic modulus of diamond and diamond-like carbon films," Thin Solid Films 228, 289 (1993).

50T. F. Page and S. V. Hainsworth, Surf. Coat. Technol. 61, 201 (1993).

51 B. O. Johansson, J. E. Sundgren, J. E. Greene et al., J. Vac. Sci. Technol. 3, 303 (1985).

52R. W. Rice, C. C. Wu, and F. Borchelt, J. Am. Ceram. Soc. 77, 2539 (1994).

53 D. Tabor, J. Inst. Metals 79, 1 (1951).

54D. M. Marsh, Proc. R. Soc. London, Ser. A 279, 420 (1964).

55T. Mura, N. Yamashita, T. Mishima et al., Int. J. Eng. Sci. 27, 1 (1989).

56J. Robertson, "Mechanical Properties and Coordinations of Amorphous Carbons," Phys. Rev. Lett. 68 (2), 220 (1992).

57. Humble and R. H. J. Hannink, Nature (London) 273, 37 (1978).

58C. A. Brookes, Nature (London) 332, 139 (1988).

59 J. Gilman, Science 261, 1436 (1993).

$60 \mathrm{~J}$. Gilman, in Mechanical Behavior of Diamond and Other Forms of Carbon, edited by M. D. Drory, D. B. Bogy, M. S. Donley et al. (Materials Research Society, Pittsburgh, 1995), Vol. 383, pp. 281.

61 For example, we have observed that high $\mathrm{cBN}$ content films deposited by ionassisted pulsed laser deposition can be much softer than the expected bulk value. hBN particulates from the deposition process are a possible culprit . 62 G. F. Cardinale, P. B. Mirkarimi, K. F. McCarty et al., Thin Solid Films 253, 130 (1994). 63P. B. Mirkarimi, (unpublished).

64X. Chu and S. A. Barnett, J. Appl. Phys. 77, 4403 (1995).

65P. B. Mirkarimi, S. A. Barnett, K. M. Hubbard et al., J. Mater. Res. 9, 1456 (1994). 


\section{Growth and characterization of BN thin films by pulsed laser deposition - application of X-ray reflectivity to optimize film growth.}

\subsection{Introduction}

X-ray reflectivity has been used to study the density, thickness and roughness of the layered structure of BN thin films as a function of growth conditions during ion-assisted pulsed laser deposition. A two layer model fit to the data shows that a low density, rough layer forms at the substrate, followed by a high density, smooth layer, where the density of each layer is sensitive to the ion energy and the ion/atom ratio used during growth. Films with the bulk density of $3.5 \mathrm{~g} / \mathrm{cm}^{3}$ are observed for $200 \mathrm{eV}$ ion energy and an ion/atom ratio of 1 , while lower density films indicative of mixed turbostratic $B N(t B N)$ and cubic $B N(c B N)$ phases occur for non-optimal growth conditions. Estimates of the cBN fraction based on density and thickness measurements agree with those determined from Fourier transform infrared spectroscopy. Transmission electron microscopy confirms this layered structure, showing an initial oriented rough $\mathrm{tBN}$ layer followed by columnar, oriented $\mathrm{cBN}$ grains extending to the film surface.

Cubic boron nitride ( $\mathrm{cBN}$ ) thin films have the potential to be used in applications requiring high hardness ${ }^{1,2}$, wide-bandgap $2-4$, or high thermal conductivity. 2 Thin films grown on $\mathrm{Si}$ substrates by ion assisted deposition techniques typically form a layered structure consisting of a thin amorphous layer at the film/substrate interface, followed by an oriented turbostratic $\mathrm{BN}$ (tBN) layer upon which the cBN phase nucleates. ${ }^{5-8}$ The crystalline quality, hardness and adhesion of the film depend sensitively upon the growth conditions. Fourier transform infrared spectroscopy (FTIR) is often used to estimate the relative fractions of tBN and $\mathrm{cBN}$ within a given film $7,9,10$, although it gives no information about their distributions. Cross-sectional transmission electron microscopy (TEM) has determined the layered structure discussed above $5-8,11$, but is difficult to use for routine characterization. However, $x$-ray reflectivity is a non-destructive technique requiring minimal sample preparation that accurately measures the density, thickness and roughness of layered thin films. In this Letter we describe how the density of BN layers as measured by X-ray reflectivity evolves as a function of growth conditions. Estimates of the $\mathrm{cBN}$ fraction based on density measurements correlate with those determined by FTIR. TEM images confirm the layered structure discussed above and show columnar (111) in-plane oriented $\mathrm{cBN}$ grains extending to the film surface under optimized growth conditions.

\subsection{Experimental}

The BN films studied here were grown by ion-assisted pulsed laser deposition. ${ }^{12}$ The excimer laser $(248 \mathrm{~nm}, \mathrm{KrF}$ ) was operated at a $45 \mathrm{~Hz}$ repetition rate and high fluence (> $90 \mathrm{~J} / \mathrm{cm}^{2}$ ) using an oscillator pumped amplifier configured in the power mode. The beam was focused onto a rotating pyrolytic boron nitride target and the ablated species were collected onto a Si (100) substrate (p-type B doped to $0.1 \Omega-\mathrm{cm}$, HF dipped to remove the native oxide) which was resistively heated to $450^{\circ} \mathrm{C}$. The substrate was simultaneously irradiated with $100 \%$ nitrogen ions from a broad beam Kaufman ion source $\left(30^{\circ}\right.$ angle of incidence from the substrate normal) where the ion energy was varied from $200-1500 \mathrm{eV}$ and the ion current adjusted $\left(128-200 \mu \mathrm{A} / \mathrm{cm}^{2}\right)$ to give a ion/atom arrival ratio of 0.6 to 1.0 . The atom arrival rate is determined by measuring the thickness of a region shadowed from the ion beam but exposed to the target flux and was approximately $0.03 \AA /$ pulse or $1.2 \AA /$ sec. Film growth was investigated as a function of ion energy for a fixed ion/atom ratio of $0.82-0.89$ and as a function of ion/atom ratio for a fixed ion energy of $200 \mathrm{eV}$.

$\mathrm{X}$-ray reflectivity has been used extensively to analyze the structure of layered thin films as has been described in detail elsewhere. 13-15 Briefly, the intensity of specularly 
reflected x-rays depends upon the electron density at and near the film surface. 16 Above the critical angle for total external reflection, the x-rays penetrate and interfere constructively or destructively with reflections from buried interfaces. The observed interference fringes can be simulated using Fresnel recursive relations modified to include interface roughness. 13,17. A non-linear least squares fit of the calculated intensity to the measured intensity determines the electron density, thickness $t$, and roughness $\sigma$ of each layer. ${ }^{13,17}$ The mass density $\rho$ is then deduced using the stoichiometry of the individual layers. ${ }^{13}$ Our measurements were performed on a four-circle Philips X-Pert MRD diffractometer using $\mathrm{Cu} \mathrm{K} \alpha$ radiation from a fixed anode line source. A parallel plate collimator, $100 \mu \mathrm{m}$ slit and flat graphite monochrometer defined the reflected beam. The sample height was adjusted to bisect the beam using a stage capable of 0.1 micron steps. A $\theta-2 \theta$ scan was performed to measure the specularly reflected intensity.

Transmission FTIR spectroscopy was used to characterize the local vibrational B-N bonding modes. Crystalline hexagonal $\mathrm{hBN}$ or $\mathrm{tBN}$ have two characteristic absorption peaks at 1370 and $800 \mathrm{~cm}^{-1}$ corresponding to the B-N in-plane stretching mode and the out-of-plane B$\mathrm{N}-\mathrm{B}$ bending mode respectively. $18 \mathrm{cBN}$ has an IR-active TO mode at $1065 \mathrm{~cm}^{-1} .19$ Simulations have shown that estimates of the cBN fraction accurate to within $5 \%$ can be obtained by taking the ratio of the peak height at $1065 \mathrm{~cm}^{-1}$ relative to the sum of the peak heights at $1065 \mathrm{~cm}^{-1}$ and $1370 \mathrm{~cm}^{-1} .12$ This procedure is commonly used to estimate the cBN fraction in thin films. $12,20,21$

The microstructure of the films was investigated using both dark field and high resolution TEM. A thin Au layer was deposited on the surface before embedding in epoxy in order to protect and clearly delineate the original cBN surface. Cross-sections were prepared with a glue line between two film surfaces. Mechanical thinning to $5-10 \mu \mathrm{m}$ was accomplished using a tripod polisher. Grazing angle ion milling resulted in uniform thin sections over large areas. Images were obtained using both a conventional JEOL 1200EX (120 $\mathrm{kV}$ ) and JEOL $2010(200 \mathrm{kV})$ high resolution microscope. Selected area diffraction patterns were taken along the $\mathrm{Si}$ [110] zone using the pattern from the underlying $\mathrm{Si}$ substrate to define the tilt angle relative to the substrate normal.

A typical x-ray reflectivity scan is shown in Fig. 4-1 (a) where the symbols show the data and the solid line shows the simulation. In order to fit the data, a two-layer model consisting of a low density layer at the film/substrate interface with roughness comparable to the film thickness and a smooth ( $3 \AA$ rms roughness) higher density layer above the first was applied in all simulations. The simulation for this sample, grown at $200 \mathrm{eV}$ ion energy and an ion/atom ratio of 0.89 resulted in $\rho=1.9 \mathrm{~g} / \mathrm{cm}^{3}$ and $t=$ $90 \AA$ for the layer at the film/substrate interface and $\rho=3.2 \mathrm{~g} / \mathrm{cm}^{3}$ and $t=850 \AA$ for the layer at

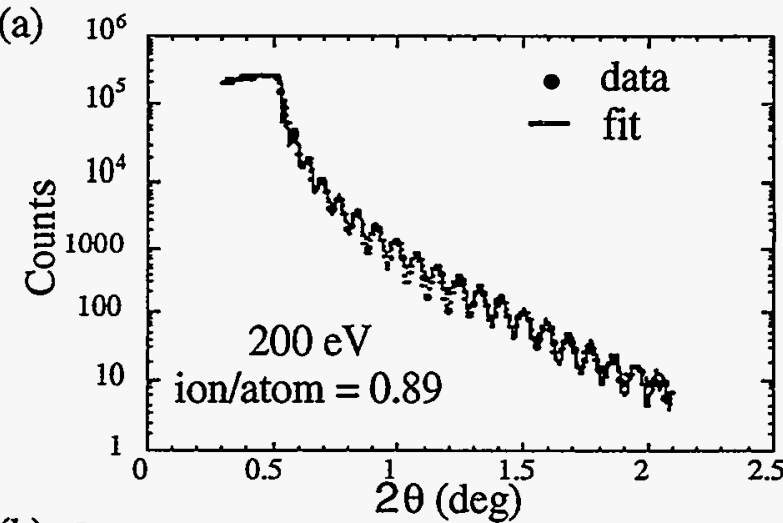

(b)

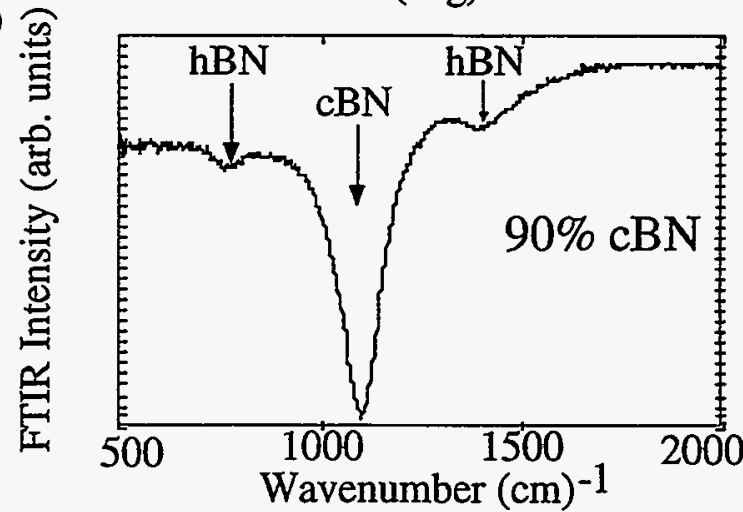

Fig. 4-1. (a) X-ray reflectivity data and fit for sample grown at $200 \mathrm{eV}, 0.89$ ion/atom ratio. (b) Transmitted FTIR spectra for same sample showing predominantly $\mathrm{cBN}$ with small $\mathrm{hBN}$ components.

the surface. By comparison, bulk $\mathrm{cBN}$ has a density of $3.47 \mathrm{~g} / \mathrm{cm}^{3}$ and bulk hBN has a density of $2.25 \mathrm{~g} / \mathrm{cm}^{3}$. The fact that the simulation results in densities lower than the bulk values indicates that the initial layer, consisting of amorphous plus highly disordered $\mathrm{tBN}$ phase, has a 
lower density than the hexagonal phase, while the upper $\mathrm{cBN}$ layer is likely mixed with a small fraction of $\mathrm{tBN}$ as expected for non-optimal growth conditions. Because the reflectivity is modeled as two uniform distinct layers, (assuming the initial $\sim 2 \mathrm{~nm}$ amorphous layer has a density comparable to the following $t B N$ layer) the slight discrepancy between the data and the simulation likely arises from the intermixing of the two phases.

Density measurements of each layer as a function of ion energy for fixed ion/atom ratio of $0.82-0.89$ are shown in Fig. 4-2(a) and those as a function of ion/atom ratio for fixed ion energy of $200 \mathrm{eV}$ are shown in Fig. 4-2(b). The density of the layer at the film/substrate interface shows no monotonic dependence on deposition conditions and is comparable to the bulk $\mathrm{hBN}$ value except for the lowest ion/atom ratio of 0.63 where $c B N$ growth is not favored. The density of the
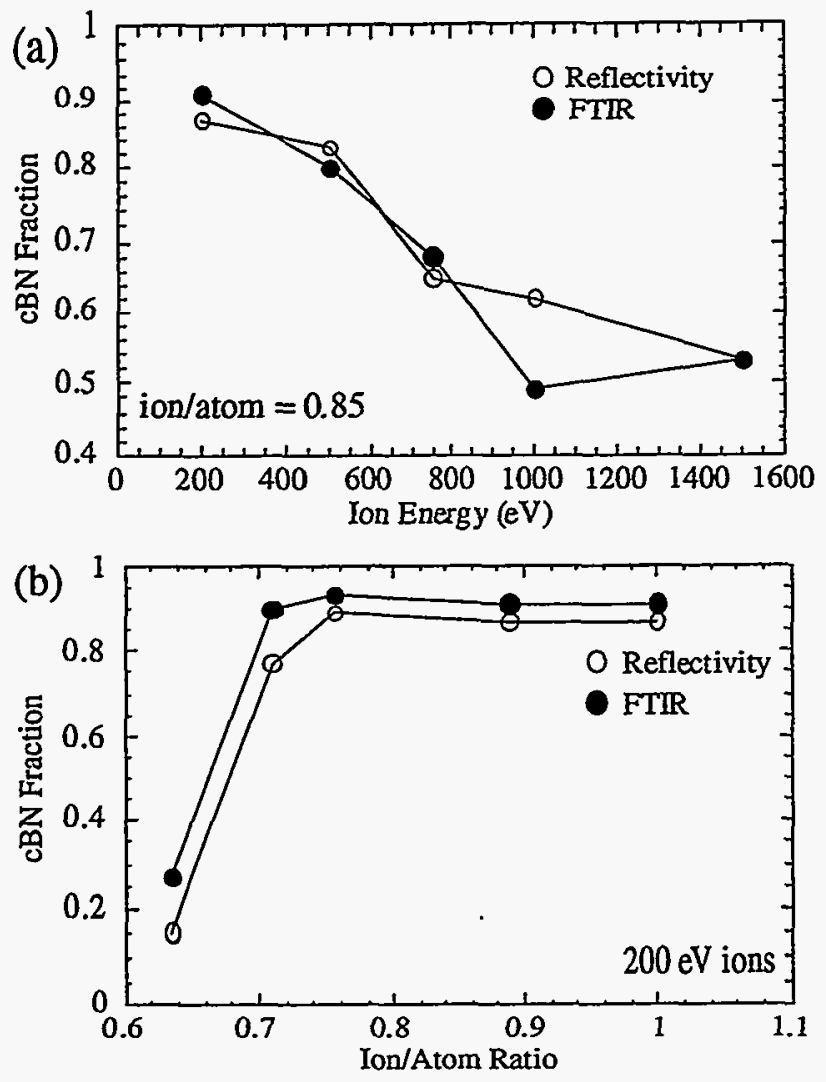

Fig. 4-3. Comparison of $\mathrm{cBN}$ fraction estimated from reflectivity measurements (O) to that estimated from FTIR spectra (0) as a function of ion energy (a) and ion/atom ratio (b).
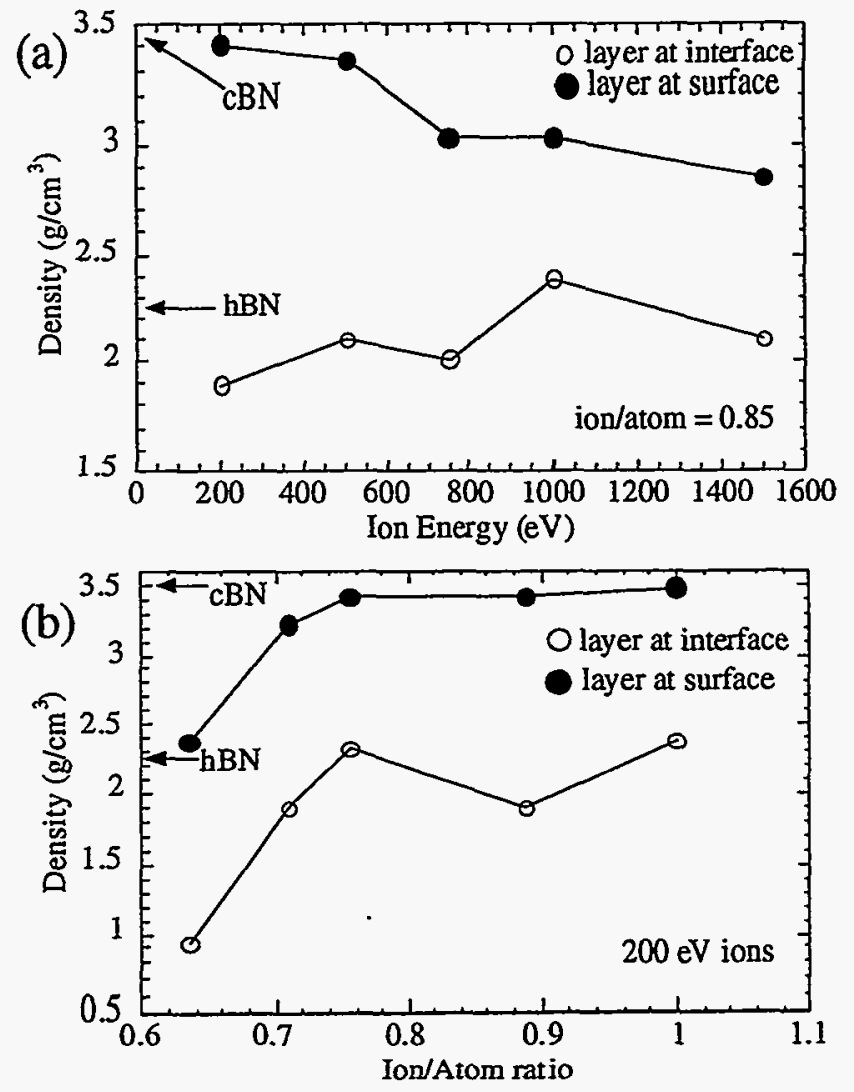

Fig. 4-2. Density of $\mathrm{cBN}$ and $\mathrm{tBN}$ layers as a function of ion energy (a) and ion/atom ratio (b). Bulk density values are indicated for comparison.

layer extending to the film surface increases monotonically towards the bulk $\mathrm{cBN}$ value as the ion energy decreases. The plateau in density for the $\mathrm{CBN}$ layer near the bulk value for films grown with $200 \mathrm{eV}$ ion energy indicates that fully dense cBN films can be grown over a wide range of ion/atom ratios.

An FTIR spectrum for the sample grown at $200 \mathrm{eV}, 0.89$ ion/atom ratio is shown in Fig. 4-1 (b). An estimate of $90 \%$ $\mathrm{cBN}$ is obtained using the procedure described above, where the background level is subtracted by extrapolating from the high wavenumber data above $1600 \mathrm{~cm}^{-1}$. In order to compare the estimate of $\mathrm{cBN}$ fraction as determined by FTIR with that determined by $x$-ray reflectivity we can apply the method of mixtures 22 to the $\mathrm{cBN}$ layer reflectivity data using the equation $1 / p$ $=(1-x) / \rho_{h B N}+x / \rho_{c B N}$, where $\rho$ is the measured density of the top layer, $\rho \mathrm{hBN}$ and $\rho_{C B N}$ are the bulk densities and $x$ is the $c B N$ 
fraction of the top layer. The $\mathrm{cBN}$ volume fraction of the total film thickness is then $\mathrm{y}=$ $\mathrm{xt}_{\mathrm{cBN}} /\left(\mathrm{t}_{\mathrm{CBN}}+\mathrm{thBN}_{\mathrm{B}}\right)$. Fig. 4-3 shows a comparison of the estimates based on reflectivity $(\mathrm{y}=$ open symbols) with those based on FTIR (closed symbols) for films grown as a function of ion energy at fixed ion/atom ratio of $0.82-0.89$ and as a function of ion/atom ratio for fixed ion energy of $200 \mathrm{eV}$, Fig. 4-3(b). We find that the estimates typically agree to within a few percent, and although the largest difference is $13 \%$, it is still within the range expected for such estimates.

In contrast to results obtained using low laser fluence and noble gas $/ \mathrm{N}_{2}$ mixtures 23, we find higher quality $\mathrm{cBN}$ films (i. e. as measured here by an increase in density and higher $c B N$ fraction), at the lowest ion energies for ion/atom ratios near 1 . Previous studies proposed that $\mathrm{cBN}$ nucleation is favored as the total ion momentum per deposited atom increases, until a resputtering regime is reached. 23 We speculate that the higher laser fluence used in this study can effectively increase the total momentum/deposited atom because the ablated species have higher energies, thus reducing the apparent ion/atom ratio required for $\mathrm{cBN}$ formation. In addition, higher laser fluence should result in more complete dissociation of the ablated species, reducing particulate incorporation and therefore allowing uninterrupted growth of $\mathrm{cBN}$ grains. Furthermore, $\mathrm{cBN}$ formation may be shifted to lower ion energies at ion/atom ratios near 1 because the higher laser fluence combined with $100 \%$ N2 ions result in higher quantities of activated growth species.

Dark-field TEM images were used to confirm the layered structure as determined by $x$-ray reflectivity. Fig. 4-4 shows a cross-section of a fully dense film grown at $200 \mathrm{eV}$ with an ion/atom ratio of 1.0. The inset in Fig. 4-4 (b) shows the selected area diffraction pattern for the entire film thickness plus some of the $\mathrm{Si}$ substrate. The pattern of sharp spots is the [110] zone-axis pattern from the $\mathrm{Si}$ substrate, while the weaker rings and arcs are from the film. In Fig. 4-4 (b) the tBN phase is illuminated using the diffuse (002) diffracted beam circled and labeled in the inset. The objective aperture size was small enough to exclude other diffracted beams from the film. This phase appears only at

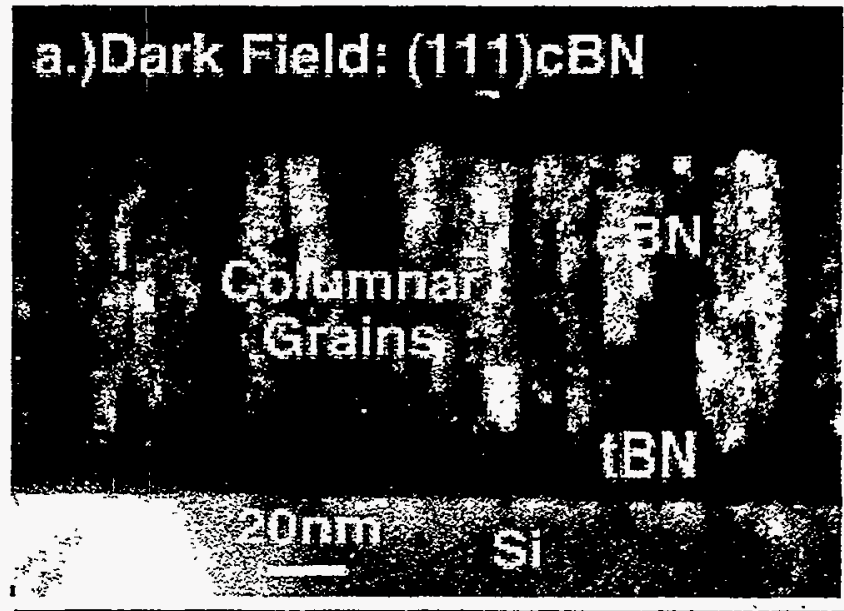

\section{b.) Dark Field: (220)cBN}
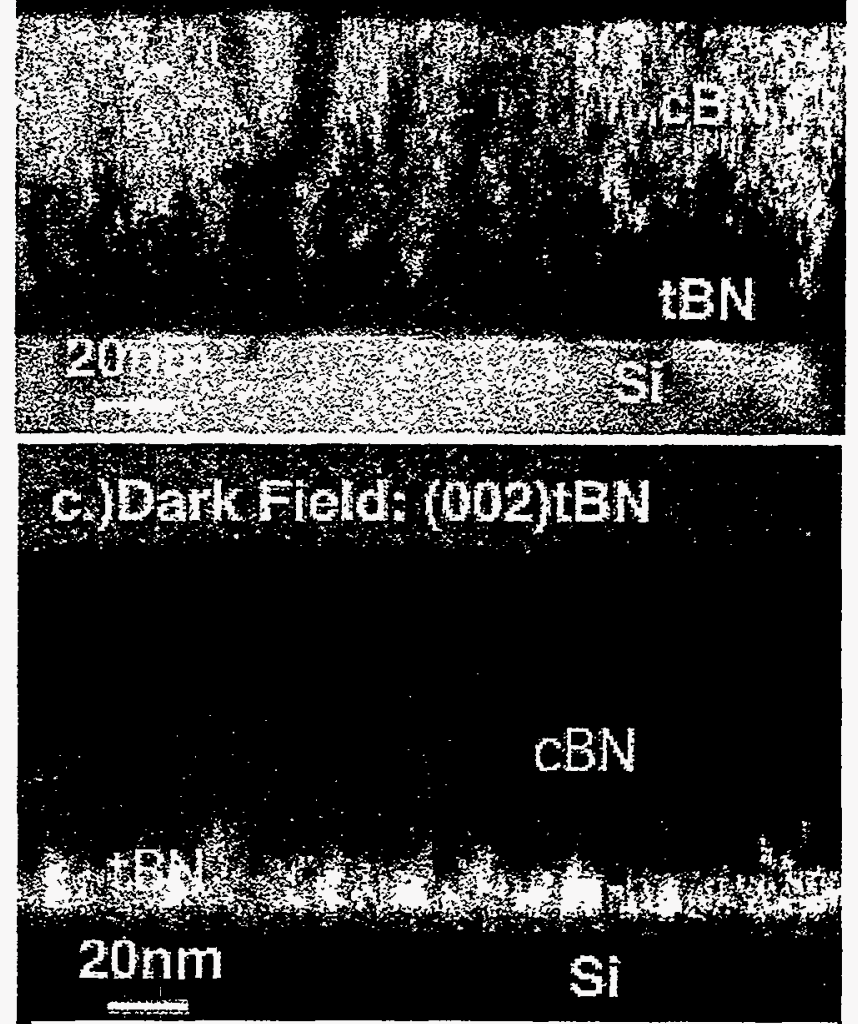

Fig. 4-4. Dark-field cross-sectional TEM images taken with (a) (111) cBN diffracted beam, (b) (200) tBN diffracted beam and (c) (220) cBN diffracted beam. Inset shows diffraction pattern and aperture positions for dark field images with labeled reflections. 
the film/substrate interface, and shows a roughness comparable to its thickness as also found with $\mathrm{x}$-ray reflectivity. The diffraction pattern shows relatively sharp arcs indexing to $\mathrm{cBN}$ that align with Si reflections. The image in Fig. 4-4a was obtained using the (111) cBN arc aligned with (220) $\mathrm{Si}$, and shows $\sim 20 \mathrm{~nm}$-wide columnar grains extending across most of the film. Also seen is a (220) cBN reflection positioned near the (004) Si reflection, i.e., the (220) cBN planes are parallel to the Si surface. This alignment is consistent with the same crystal orientation just mentioned for (111) cBN; assuming that one crystallographic alignment rule accounts for all the aligned reflections, the rule is: $(220) \mathrm{cBN}$ parallel to (004)Si, and (111)cBN parallel to (220) Si.

This alignment would have two variants, i.e., there are two independent and equivalent $<220>$ directions in the (004) surface plane of the Si. Thus dark-field imaging with the (111) $\mathrm{cBN}$ arc illuminates only $\sim 1 / 2$ of the columnar grains. However, the $(220) \mathrm{cBN}$ reflection is common to both variants, and dark-field imaging with it illuminates almost all the upper part of the film with columnar grains, as seen in Fig. $4-4$ (c). Careful examination of the diffraction pattern shows weak rings indexing to (111) and $(220) \mathrm{cBN}$, indicating that some $\mathrm{cBN}$ crystallites are not aligned as above. Dark-field imaging with these rings illuminates isolated grains in the upper part of the film; such grains probably account for the remaining dark regions in the upper layer seen in Fig. $4-4$ (c). These misaligned crystallites and the angular
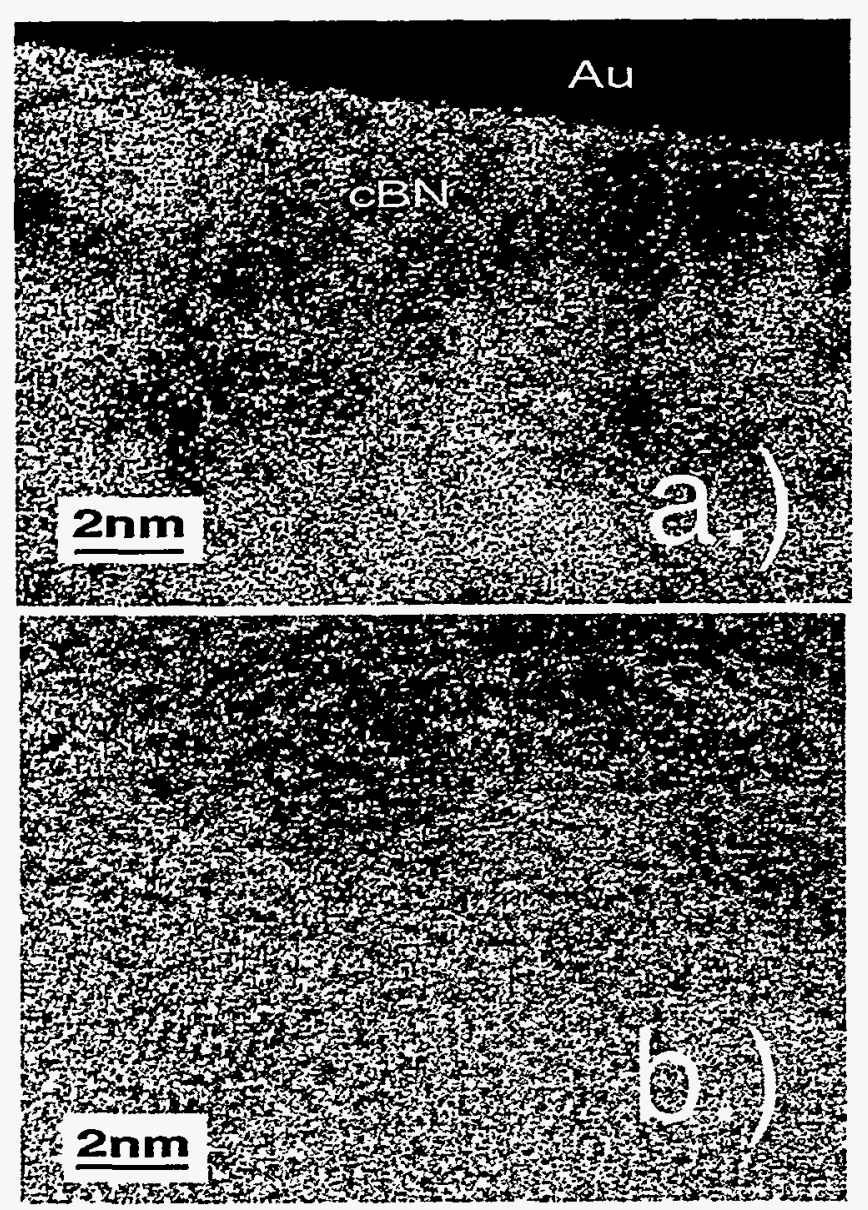

Fig. 4-5. High resolution TEM images of the film surface covered by Au layer for protection (a) showing $\mathrm{cBN}$ lattice fringes within a few atomic layers of the surface and (b) the cBN/tBN interface showing $\mathrm{cBN}$ nucleation from oriented (002) $\mathrm{tBN}$ planes. width of the aligned $\mathrm{cBN}$ arcs lead us to refer to the above alignment rule as "preferred" but not exact. These dark-field images confirm the X-ray reflectivity results, namely that this film, grown under optimal conditions, consists of a nearly full density $\mathrm{cBN}$ surface layer which grows from a thin, rough $\mathrm{tBN}$ layer.

Lattice fringes from both the $\mathrm{cBN}$ and $\mathrm{tBN}$ phases are observed in the high resolution images shown in Fig. 4-5. The $\mathrm{cBN}$ lattice fringes shown in Figure 4-5 (a) indicate that $\mathrm{cBN}$ grains grow to within a few atomic layers of the surface, whereas previous reports have indicated the presence of a hexagonal phase at the surface 24 . Our results suggest that the surface phase is sensitive to the growth conditions, since the lower ion energy (200 $\mathrm{eV}$ used here may minimize disorder at the surface of the growing film. Fig. 4-5 (b) shows the interface between the $\mathrm{tBN}$ layer and the $\mathrm{cBN}$ layer. As reported in previous work $5-8,11$, we find that $\mathrm{CBN}$ (111) planes nucleate preferentially from the oriented tBN (002) planes, which leads to their alignment with respect to the Si substrate.

In summary, we've shown that $x$-ray reflectivity can be used to analyze the layered structure of $\mathrm{BN}$ thin films, giving a quantitative measure of the density, thickness and roughness of each layer. We find the $c B N$ layer density to be sensitive to ion energy and ion/atom ratio used during film growth, where fully dense $c B N$ layers 
are realized for the lowest ion energy $(200 \mathrm{eV})$ and the highest ion/atom ratios explored in this study. Estimates of the $\mathrm{cBN}$ fraction based on density and thickness measurements agree with those derived from accepted methods based on FTIR spectroscopy. Dark field and highresolution TEM confirm the layered structure and show that when optimal growth conditions are employed, columnar (111) in-plane $\mathrm{CBN}$ grains grow to within a few atomic layers of the surface.

\subsection{References}

${ }_{1}^{1}$ H. Holleck, J. Vac. Sci. Technol. A 4, 2661 (1986).

2L. Vel, G. Demazeau, and J. Etourneau, Mater. Sci. Eng. B 10, 149 (1991).

3R. C. DeVries, GE CRD Report No. 72CRD178 (1972).

${ }_{5}^{4}$ S.P.S. Arya and A. D'Amico, Thin Solid Films 157, 267 (1988).

5D. J. Kester, K. S. Ailey, R. F. Davis, and K. L. More, J. Mater. Res. 8, 1213 (1993).

${ }^{6}$ A. K. Ballal, L. Salamanca-Riba, C. A. Taylor, II, and G. L. Doll, "Structural characterization of preferentially oriented cubic BN films grown on Si (100) substrates," Thin Solid Films 224, 46 (1993).

7D. R. McKenzie, W. D. McFall, W. G. Sainty, C. A. Davis, and R. E.Collins, Diam. Relat. Mater. 2, 970 (1993).

8D. R. McKenzie, D. A. Muller, E. Kravtchinskaia, D. Segal, D. J. H. Cockayne, G. Amaratunga, and R. Silva, "Synthesis, structure and applications of amorphous diamond," Thin Solid Films 206, 198 (1991).

9 Daniel J. Kester and Russell Messier, "Momentum/atom," J. Appl. Phys. 72, 504 (1992).

10M. Okamoto, Y. Utsumi, and Y. Osaka, Plasma Sources Sci. Technol. 2, 1 (1993).

${ }^{1}$ D. L. Medlin, T. A. Friedmann, P. B. Mirkarimi, G. F. Cardinale, and K.F. McCarty, J. Appl. Phys. 79, 3567 (1996).

12T. A. Friedmann, P. B. Mirkarimi, D. L. Medlin, K. F. McCarty, E. J. Klaus, D. R. Boehme, H. A. Johnsen, M. J. Mills, D. K. Ottesen, and J. C. Barbour, "Ion-assisted pulsed laser deposition of cubic boron nitride films," J. Appl. Phys. 76 (5), 3088 (1994).

13 M. F. Toney and Sean Brennan, J. Appl. Phys. 66, 1861 (1989).

14 C. A. Lucan, T. D. Nguyen, and J. B. Kortright, Appl. Phys. Lett. 2, 101 (1991).

15Y. Huai, M. Chaker, J. N. Broughton, E. Gat, and H. Pepin, Appl. Phys. Lett. 65, 830 (1994).

16J. Als-Nielsen, in Structure and Dynamics of Surfaces, edited by W. Schommers and P. von Blanckenhagen (Springer, Berlin, 1987), Vol. II, pp. 181.

${ }_{17} \mathrm{M}$. Born and E. Wolf, Principles of Optics (Pergamon, Oxford, 1970).

18R. Geick, C. H. Perry, and G. Rupprecht, Phys. Rev. 146, 543 (1966).

19P. J. Gielisse, S. S. Mitra, J. N. Plendl, R. D. Griffis, L. C. Mansur, R. Marshall, and E. A. Pascoe, "FTIR on bulk cBN," Phys. Rev. 155, 1039 (1967).

${ }^{20}$ T. Wada and N. Yamashita, J. Vac. Sci. Technol. A 10, 515 (1992).

${ }^{21}$ K. Inagawa, K. Watanabe, H. Ohsone, K. Saitoh, and A. Itoh, J. Vac. Sci. Technol. A 5, 2696 (1987).

22 J. J. Cuomo, D. L. Pappas, J. Bruley, J. P. Doyle, and K. L. Saenger, "Vapor Deposition processes for amorphous carbon films with sp3 fractions approaching diamond," J. Appl. Phys. 70 (3), 1706 (1991).

23P. B. Mirkarimi, K. F. McCarty, D. L. Medlin, W. G. Wolfer, T. A.Friedmann, E. J. Klaus, G. F. Cardinale, and D. G. Howitt, J. Mater. Res. 9, 2925 (1994).

${ }^{24}$ K.S. Park, D. Y. Lee, K. J. Kim, and D. W. Moon, Appl. Phys. Lett. 70, 315 (1997). 


\section{Scaling theory of the Hall-Petch relation for multilayers}

\subsection{Introduction}

This work is one piece of the puzzle towards developing a dislocation based theory of flow in multilayers . Our approach was to develop models appropriate to an ideal, epitaxial multilayer, and then modify the models to be more appropriate for "real" multilayers. To date, we have developed an algorithm, within isotropic elasticity theory, for calculating the exact stress field (i.e. including all images) arising from a dislocation within a multilayer. Figure 5-1 displays the stress field due to a dislocation present within a multilayer coating. The stress has been normalized to that stress expected from a dislocation situated at the identical distance, but within an infinite, uniform bulk sample. The units in figure 5-1 are chosen such that the "soft" material of the multilayer has a shear modulus of 1 , whereas the "hard" material of the multilayer has a shear modulus of 1.5. The bulk material (negative $x$-axis) has a modulus of 0.375 within the same units. The unit of length is chosen so that each bilayer has a width of 1 . Note that the multilayer does not screen significantly the stress field from the dislocation.

Koehler's theory of the strength enhancement assumes that no more than one dislocation may occupy an individual layer. However, for larger layer thicknesses, this may not be a reasonable assumption. It is therefore reasonable to investigate the properties of pileups within the individual multilayers. Armed with the solution above, we have begun the necessary investigation. We considered first the operation of a source within an individual layer. As the source operates, the stress exerted on the interface increases. In addition, the stress ahead of the pileup, but in different layers of the multilayer also becomes significant. These stresses

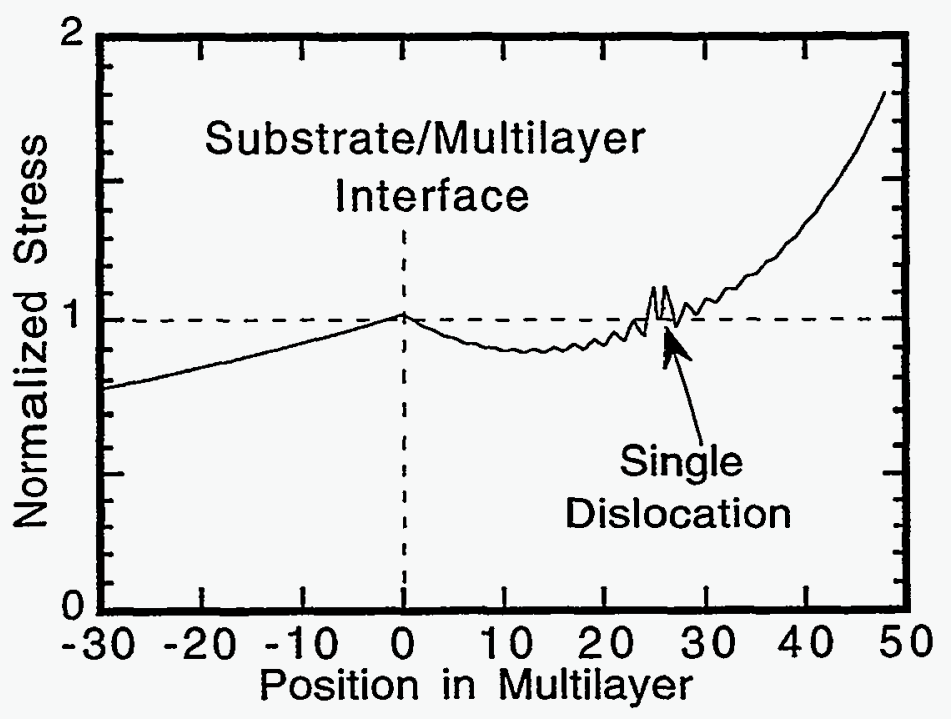

Fig. 5-1 Stress in a multilayer film with a single may be large enough to initiate in a uniform medium. operation of sources in additional layers. We have investigated this possibility, and find the following two preliminary results: 1) Operation of sources in subsequent layers can lead to a pileup which extends through many bilayers of the multilayer. 2) These extended pileups can lead to either a finite amount of plastic deformation (at low applied stresses) or to steady state flow (at higher stresses). The two regimes appear to be demarcated by a "critical stress," but the precise value of the "critical" stress depends sensitively on structural factors (e.g. variations in layer thicknesses). Both observations are relevant to modeling flow in multilayers.

A scaling theory describing the strength of lamellar materials is developed. The theory is based on the premise that dislocation pileups are responsible for the yielding behavior of the lamellar material. The theory is developed in terms of four parameters: the pileup length, the exponent characterizing the divergence of the dislocation density at the interface, a critical distance between the lead dislocation and the interface, and a pinning stress at the interface which acts as an additional hindrance to dislocation motion. 


\subsection{Background}

Lamellar materials, either man made (e.g. TiN/NbN multilayers ${ }^{1}$ ), or naturally occurring (e.g. the lamellar $\gamma$-TiA1/TiAl $1_{3}$ compounds) are being explored currently for their technological promise. In particular, it is known that under certain circumstances, one may observe yield strengths which exceed those observed in the bulk crystalline forms of either of the single components. The origins of this enhancement have been discussed widely in the literature $2-5$. A common explanation originates in the interaction of dislocations with the interfaces between elastically mismatched materials. Two major themes in these discussions are the occurrence of Koehler strengthening ${ }^{4}$ and the Hall-Petch Relation 1,6,7.

The Hall-Petch Relation 6,7 relates the yield stress, $\sigma_{y}$, of a polycrystalline solid to its grain diameter, $d$ :

$$
\sigma_{y}=K d^{-1 / 2}+\sigma_{0}
$$

where $\sigma_{0}$ is a constant offset and $K$ is a constant. The success of the Hall-Petch Relation in describing the strength of many polycrystalline materials suggests that its origin are very fundamental, and that similar behavior should be observed in multilayers.

A common explanation of the Hall-Petch Relation comes from dislocation pileup theory. In this theory, the grain boundary is assumed to act as an obstacle to dislocation motion. Dislocations emitted from a source, move along the same slip plane and propagate towards the obstacle. Once the lead dislocation is stopped by the obstacle, the trailing dislocations stop behind it due to mutual repulsion. As this dislocation pileup forms, the force exerted on the obstacle eventually exceeds its strength, and the polycrystalline materials plastically yields. In multilayers one sometimes observes pileups against interlayer interfaces ${ }^{8}$, and a pileup model may well be applicable.

The traditional development of the pileup model implicitly assumes that the elastic properties of a material are homogeneous (i.e. translationally invariant). Compositionally modulated multilayers clearly violate this assumption. However, a scaling argument, also based in pileup theory, leads to a more general scaling form applicable to multilayers:

$$
\sigma_{y}=K d^{-a}+\sigma_{0},
$$

where $a$ is possibly not equal to $1 / 2$.

The original derivation of the Hall-Petch relation, which stems from pileup theory Eq. (1), contains simplifying assumptions: linear elasticity, and that dislocations are infinitely long, perfectly straight and parallel. The derivation rests on two facts: First, the number of dislocations in a pileup, $N$, is proportional to both the applied stress, $\sigma_{A}$, and the pileup length, $L$,

$$
N \propto \sigma_{A} L .
$$

Second, the total stress on the lead or pinned dislocation, $\sigma_{\text {lead }}$, arising from $\sigma_{A}$ and the interaction stress from the trailing dislocation is given by 9

$$
\sigma_{\text {lead }}=N \sigma_{A} \text {. }
$$

Eqs. (3) and (4) give

$$
\sigma_{A} \propto \sqrt{\frac{\sigma_{\text {lead }}}{L}} .
$$


If one assumes that the obstacle blocking the lead dislocation is overcome when $\sigma_{\text {lead }}$ reaches a critical value and that the pileup length, $L$, is proportional to the grain diameter, $d$, Eq. (1) is obtained. The constant offset, $\sigma_{A}$, can be attributed to a friction stress.

The preceding argument cannot be used to describe pileups against an elastic inhomogeneity since Eq. (3) assumes implicitly that elastic properties are homogeneous. Eq. (3) is an application of the virtual work principle 9,10 . It applies to pileups in homogeneous materials because the dislocation interaction energy, $U_{\text {int }}$, is translationally invariant. Under the translation, $x \rightarrow x_{-} y$

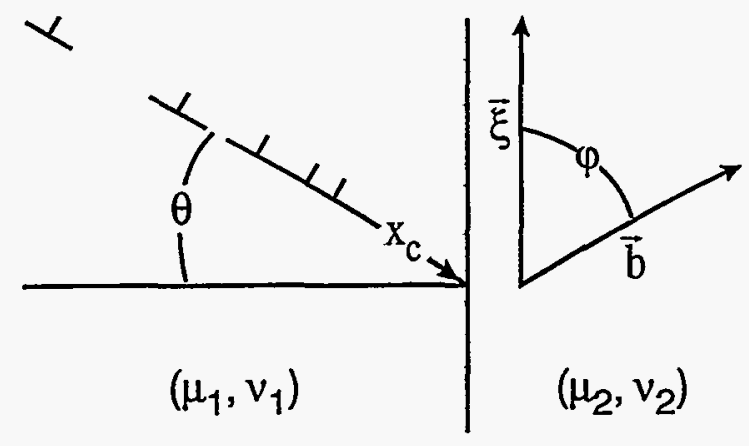

TRI-6342-6036-0

Fig. 5-2. Dislocation pileup against an elastic interface at angle $\theta$ from normal incidence, $\mu$ and $v$ are respectively the shear moduli and Poisson's ratio of the two materials. The angle, $\varphi$, between the Burgers vector, $\bar{b}$, and the line direction, 5 also parameterizes the pileup. $x_{c}$ is the critical distance for the lead dislocation.

$$
U_{\text {int }}\left(\left\{x_{i}\right\}\right)=U_{\text {int }}\left(\left\{x_{i}-y\right\}\right)
$$

where $\left\{x_{i}\right\}$ are the positions of individual dislocations. If there are elastic interfaces at coordinates $\left\{z_{i}\right\}$ (Fig. 5-2), the elastic mismatch stresses (or "image stresses") break the translational symmetry of the system:

$$
U_{\text {int }}\left(\left\{x_{i}\right\} ;\left\{z_{i}\right\}\right) \neq U_{\text {int }}\left(\left\{x_{i}-y\right\} ;\left\{z_{i}\right\}\right) .
$$

The interface positions, $\left\{z_{i}\right\}$, are not translated according to the virtual work principle because they are not dynamic variables.

\subsection{Alternative derivation of the Hall-Petch relation}

The alternate derivation of the Hall-Petch relation uses the scaling properties of dislocation pileups and in particular the scaling of the continuum pileup densities to obtain Eq. (2). In this manner, the origins of Eq. (1) are made explicit: The scaling phenomenon expressed in Eq. (1) stems solely from the fact that stresses arising from the presence of a dislocation are homogeneous (in the mathematical sense).

This homogeneity gives rise to the dilatational symmetry. The stress at position $x$ from a dislocation at position $x^{\prime}$ in a medium with elastic boundaries, $\left\{z_{i}\right\}$, and elastic constants, $\{(\mu$, $\left.v)_{i}\right\}$, (see Fig. 5-2) satisfies

$$
\sigma\left(\lambda x, \lambda x^{\prime} ;\left\{\lambda z_{i}\right\},\left\{(\mu, v)_{i}\right\}\right)=\lambda^{-1} \sigma\left(x, x^{\prime} ;\left\{z_{i}\right\},\left\{(\mu, v)_{i}\right\}\right),
$$


where $x$ and $x^{\prime}$ represent distances along a linear array of dislocations. Eq. (8) is verified easily for the simplified case of a pure screw dislocation near an elastic interface in an isotropic medium 10 .

The equilibrium density of a dislocation pileup in a multilayer is found by solving

$$
\int_{0}^{L} \sigma\left(x, x^{\prime} ;\left\{z_{i}\right\}\right) n\left(x^{\prime}\right) d x^{\prime}+\sigma_{A}=0
$$

Dependence of $\sigma\left(x, x^{\prime} ;\left\{z_{i}\right\}\right)$ on the elastic constants and Burgers vector is implicit. It is assumed that $L$, the pileup length, also characterizes the period of a multilayer. $n(x)$ can be related to the equilibrium density of a pileup in a multilayer of unit length and unit applied stress through the coordinate transformation, $\eta=x / L$.

$$
n(x)=\sigma_{A} f(x / L) ; \text { where } \int_{0}^{L} \sigma\left(\eta, \eta^{\prime} ;\left\{\zeta_{i}\right\}\right) f\left(\eta^{\prime}\right) d \eta^{\prime}+1=0
$$

and $\zeta_{i}=z_{i} / L$

The scaling formula, Eq. (2), is obtained by hypothesizing that the criteria for whether a given pileup leads to yielding are local to the head of the pileup. For the homogeneous case, the local criterion was chosen to be that the total stress on the lead dislocation must be greater than or equal to the pinning stress 6,7 . Other possibilities for yielding criteria include Koehler's criterion, that the lead dislocation approach to within its core width ${ }^{4}$, or that the stress just ahead of the pileup exceed a critical value 11 . So long as the criteria are local to the pileup head, a scaling form can be found.

The head of a dislocation pileup (located at $x=0$ ) can be characterized in the continuum dislocation model by expanding the density, $n(x)$, in a series about $x=0$, the position of the impeding elastic interface, and retaining only the most singular term:

$$
n(x)_{x \rightarrow 0}=\sigma_{A} c_{0}(x / L)^{-a}
$$

with $c_{o}$ an expansion coefficient. From Eq. (11), the density near the head of the pileup is proportional to $\sigma_{A} L^{a}$. Because the yielding criteria depend only on the local configuration, whatever criteria used can be cast, at least formally, into a critical dislocation density criterion:

$$
n(x)_{x \rightarrow 0}=n_{0} x^{-a} \text {. }
$$

Combining Eqs. (11) and (12),

$$
\sigma_{y}=\sigma_{A}=\frac{n_{0}}{c_{0}} L^{-a}
$$

The scaling exponent, $a$, is the strength of the density singularity at the head of the pileup. For the elastically homogeneous case, $n(x)$ is known $10,12,13$, and $a=1 / 2$ which agrees with the HallPetch relation, Eq. (1).

As a check of the above scaling analysis, one may compare known strengths of density singularities to exponents derived from numerical simulations of discrete dislocation pileups (similar to those of references 14,15 ). In general, it is very difficult to solve Eq. (10). However, the dislocation density for a screw dislocation pileup normal to a single elastic interface between two isotropic media was solved by Barnett 16 for which

$$
a=\frac{2}{\pi} \arcsin \left(\sqrt{\frac{1-\kappa}{2}}\right) ;
$$


where $\kappa$ is the strength of the image dislocations created by the elastic interface. For a pileup in a material of shear modulus $\mu_{1}$ against material of shear modulus $\mu_{2}, \kappa=\left(\mu_{2}-\mu_{1}\right) /\left(\mu_{2}-\mu_{1}\right)$. Fig. 5-3 displays the exponents expected from Eq. (14) and those from the numerical calculations of yield strength (not the dislocation density). In the numerical study, yielding is assumed to occur when the lead dislocation feels a critical stress while located within a critical distance of the interface. The agreement between the two values is excellent.

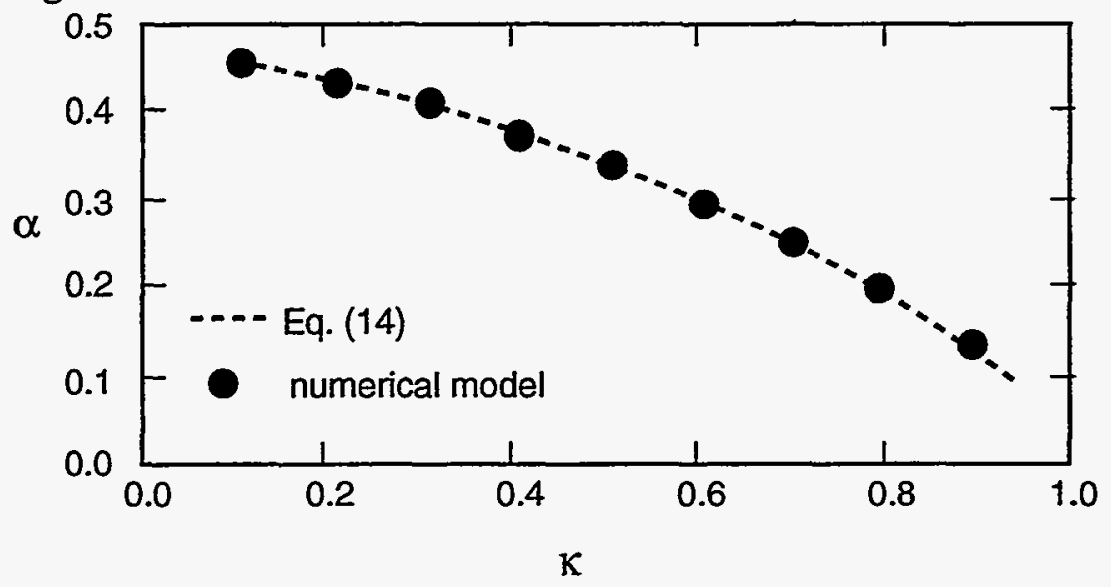

TRI-6342-6037-0

Fig. 5-3. Numerical calculation of scaling exponent, $a$, for screw dislocation pileups impinging with normal incidence upon an elastic interface (circles). Data is compared with the strength of the density singularity in the corresponding continuum model, Eq. (14).

The numerical solutions can be extended to mixed and edge oriented segments, as well as nonnormal incidence (see Fig. 5-2). The exponents $a$ obtained from numerical simulation of three cases are displayed in Fig. 5-4. The exponents display a slight dependence on orientation, but seem to lie near $a \sim 0.45$ for the chosen set of elastic constants. Experimentally determined exponents for a number of materials fall in this range 2 , though typically, the uncertainty in the measurements exceeds the differences between the value $a=1 / 2$ and the values for $a$ reported here.

Armed with the scaling form, one can draw further conclusions concerning the dependence of yield strength on specific parameters. To demonstrate how additional scaling relations may be found, the yielding criteria of Anderson and $\mathrm{Li}^{14}$ will be used. These criteria assume yielding occurs when the lead dislocation is able to penetrate the elastic interface. The relevant parameters are $x_{c}$, a critical distance from the interface which the lead dislocation must approach, and $\sigma_{p}$, a pinning stress in addition to elastic mismatch stresses which the dislocation must overcome after it reaches position $x_{c}$ (Fig. 5-2). 


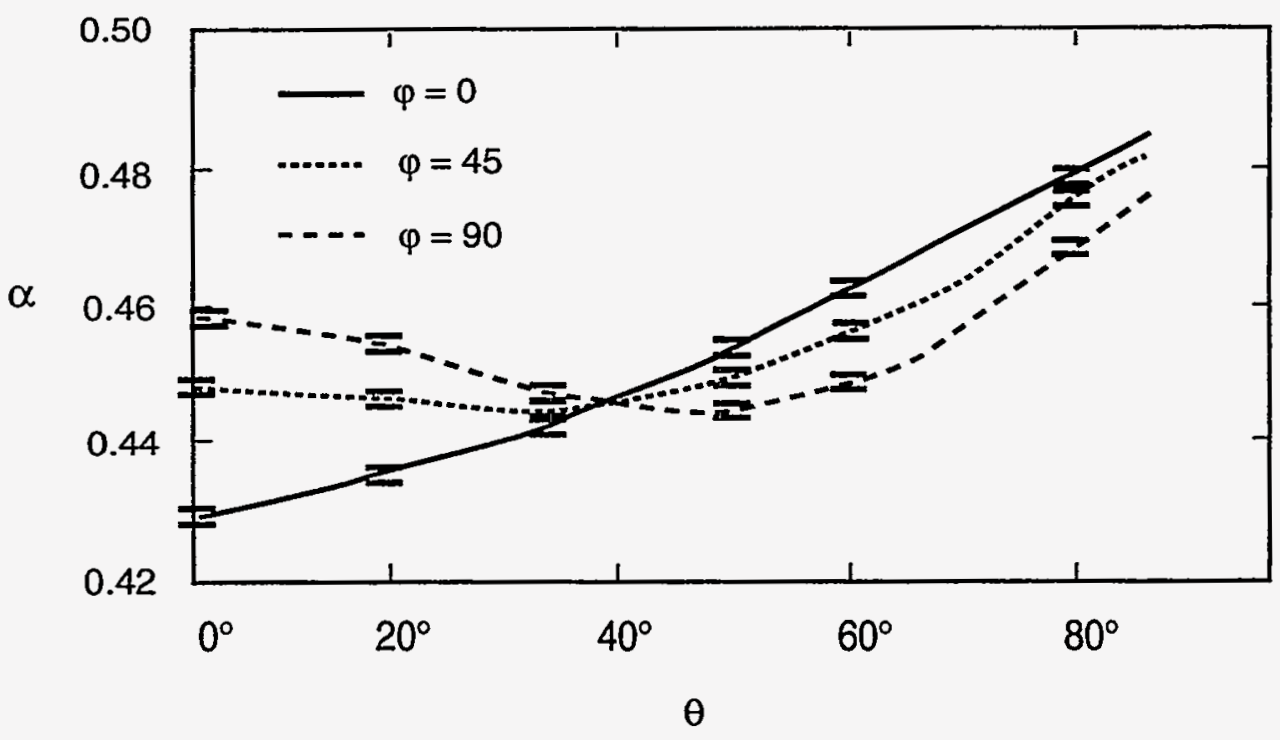

TRI-6342-6035-0

Fig. 5-4. Scaling exponents calculated using a numerical model of discrete dislocation pileups. Exponents are plotted against the pileup orientation $\theta$ for $\kappa=0.2 v_{1}=0.2$, and $v_{2}=0.4$. The three curves are for pileups of pure screw $\left(\varphi=0^{\circ}\right)$, pure edge $\left(\varphi=90^{\circ}\right)$, and mixed dislocations $\left(\varphi=45^{\circ}\right)$. Error bars indicate the typical variation in exponents one finds in fitting Eq. (13) to numerical model results.

Scaling arguments elucidate $\sigma_{y}^{\prime}$ 's dependence on $x_{c}$ and $\sigma_{p}$. Eq. (8) implies that if an array of dislocations $\left\{x_{i}\right\}$ are in equilibrium under an applied stress $\sigma_{A}$, the scale transformed positions, $\left\{x_{i}\right\} \rightarrow\left\{\lambda x_{i}\right\}$, interfaces, $\left\{z_{i}\right\} \rightarrow\left\{\lambda z_{i}\right\}$, and applied stress, $\sigma_{A} \rightarrow \lambda-{ }^{-1} \sigma_{A}$, also give an equilibrium configuration. Furthermore, any stress fields arising from such a configuration will also scale the same way. Generally, positions have scaling dimension $d_{\text {positions }}=1$, and stresses have scaling dimensions $d_{\text {stresses }}=\ldots 1$. Consequently, the general dependence of $\sigma_{y}$ on $L, x_{c}$ and $\sigma_{p}$ scales according to:

$$
\sigma_{y}\left(L, x_{c}, \sigma_{p}\right)=\lambda \sigma_{y}\left(\lambda L, \lambda x_{c}, \lambda^{-1} \sigma_{p}\right) .
$$

Ignoring the constant offset, $\sigma_{0}$, Eq. (2) gives

$$
\sigma_{y}\left(L, x_{c}, \sigma_{p}\right)=\lambda \sigma_{y}\left(\lambda L, \lambda x_{c}, \lambda^{-1} \sigma_{p}\right)
$$

Combining Eqs. (15) and (16) while setting $\lambda=x_{c}^{-1}$ and $L=x_{c}$, one finds:

$$
K\left(x_{c}, \sigma_{p}\right)=x_{c}^{-(1-a)} \sigma_{y}\left(1,1, x_{c} \sigma_{p}\right) .
$$

The combination $x_{c} \sigma_{p}$ has scaling dimension 0 . This argument can be made physically more meaningful by making it completely dimensionless. As a dislocation approaches an interface to within $x_{c}$, its self elastic mismatch stress has the form ${ }^{17}$

$$
\sigma_{l}=\frac{B}{x} \text {. }
$$


where $B$ depends on $\left\{(\mu, v)_{i}\right\}, \theta$, and $\varphi$ and the Burgers vector (see Fig. 5-2). Hence, a function with dimensionless argument can be defined, $g(\Theta)=\sigma_{y}(1,1, B \Theta)$, and 18

$$
\sigma_{y}\left(L, x_{c}, \sigma_{p}\right)=L^{-a} x_{c}^{-(1-a)} g\left(\sigma_{p} / \sigma_{I}\right) .
$$

The undetermined function, $g(\Theta)$, can be found using:

$$
g(\Theta)=\left(\frac{L}{x_{c}}\right)^{a} \sigma_{y}\left(L / x_{c}, 1, B \Theta\right) .
$$

where numerical simulations of discrete dislocations give $\sigma_{y}\left(L / x_{c}, 1, B \Theta\right)$. Eq. (20) is derived from Eq. (19) using Eq. (15) and setting $\lambda=x_{c}^{-1}$.

Eq. (20) indicates that $y(\Theta)$ should be independent of the ratio $L / x_{c}$ used in the numerical calculation. This independence or collapse is tested in Fig. 5-5 for a single interface between two isotropic media. The proposed scaling form works best for large pileups, $L / x_{c} \geq 100$ : The continuum approximation is only valid for large pileups.

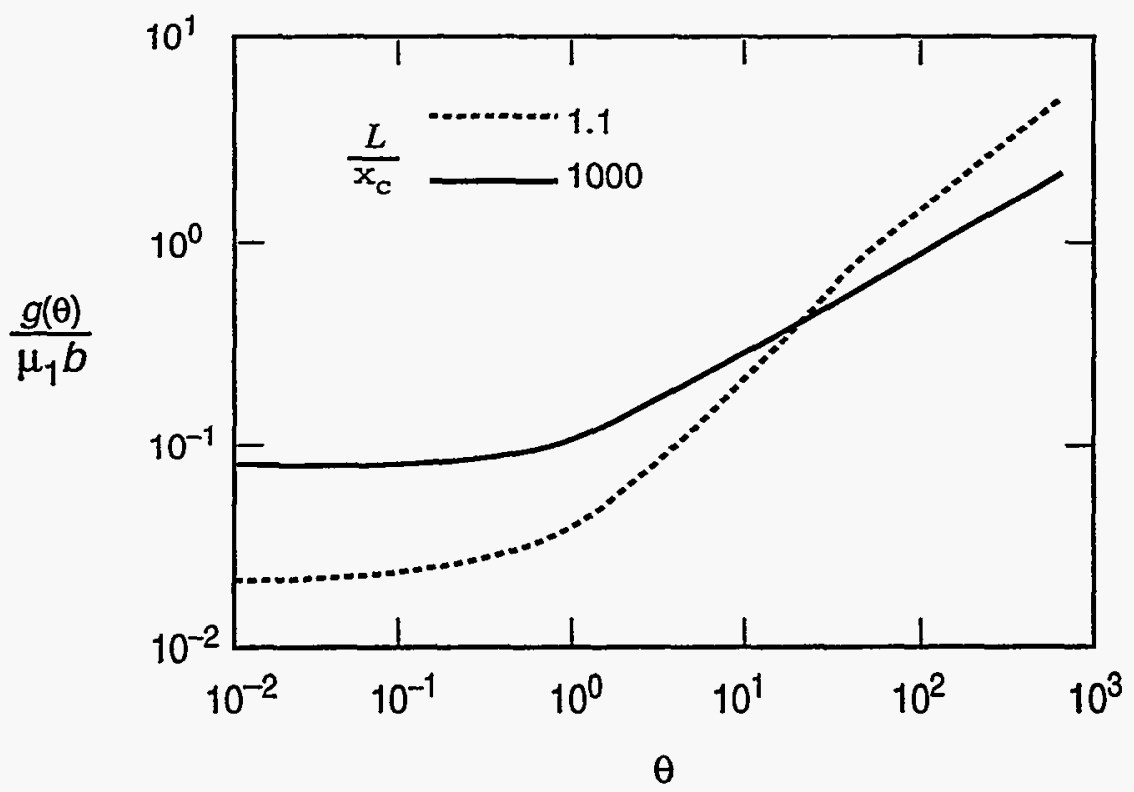

TRI- $\$ 42-6038-0$

Fig. 5-5. $g(\Theta) /\left(\mu_{1} b\right)$ plotted against $\Theta(b=$ magnitude of Burgers vector. Plots were obtained using Eq. (19), for various values of the ratio $L / x_{c}$. Parameters are $K=0.2, v_{1}=0.2, v_{2}==0.4$, and $\theta=45^{\circ}$, and $\varphi=90^{\circ}$.

In principle, Eq. (19) can be used to analyze hardness vs. layer thickness data. Presently, it is unclear how microstructural features such as grain size combine with compositional wavelength to determine the yield strength of lamellar materials ${ }^{2}$. To avoid this complication, Vicker's hardness data from single crystal TiN/NbN multilayer films 1,19 are used to illustrate the analysis.

A single interface approximation is used for the numerical analysis. In the NbN/TiN multilayers, it is assumed that pileups in NbN layer form in the $\{110\}<110>$ slip system against the NbN/TiN interface and are responsible for yielding. The Voigt averaged isotropic elastic moduli are used for numerical calculation of the scaling exponent, $a$, and the scaling function, 
$g(\Theta):(\mu, v)_{\mathrm{NoN}}=(156 \mathrm{GPa}, .270),(\mu, v)_{\mathrm{TiN}}=(190 \mathrm{GPa}, .251)$, giving $\kappa=0.098$, and $a=0.48$. Pileup lengths, $L$, are assumed to be half the distance between interfaces along the slip plane in a $\mathrm{NbN}$ layer. Assuming that the pinning distance, $x_{c} \approx 2 b^{4}, \sigma_{I} \approx .7 \mathrm{GPa}$. The value of the scaling function, $g(\Theta)$ is found by fitting Eq. (19) to the hardness $(H)$ vs. multilayer wavelength $(\Lambda)$ data 1 while using appropriate geometric factors and assuming the empirical relationship: $H \approx 3 \sigma_{y}$. One finds that the ratio of the pinning stress to the image stress (Koehler strengthening ${ }^{4}$ ) is approximately 19 , and $\sigma_{p} \approx 14 \mathrm{GPa}$.

\subsection{Conclusion}

In conclusion, a scaling form for the yield strength of multilayers has been derived, thereby extending formally the application of the Hall-Petch relation to lamellar materials. The form has been verified numerically for the limited case of a pileup against a single interface between two isotropic media. the dislocation pileup based theory predicts that the strength of a multilayer should scale as $L^{a}$, where $L$ is the nominal length of the pileup, and $a$ is the exponent characterizing the divergence of the dislocation density at the interface. It has also been demonstrated that scaling arguments can be used to determine dependences of hardness on additional parameters which one might use for yielding criteria. Here, this dependence has been given for a set of yielding criteria which incorporates Koehler strengthening and allows for additional stresses opposing dislocation motion across an interlayer interface.

The authors gratefully acknowledge P.B. Mirkarimi for insightful conversations and the financial support of Sandia National Laboratories.

\subsection{REFERENCES}

1M. Shinn, L. Hultman, and S.A. Barnett, Journal of Materials Research 7, 901 (1992).

2G.S. Was and T. Foecke, Thin Solid Films 286, 1 (1996).

3 S.A. Barnett and M. Shinn, Annu. Rev. Mater. Sci. 24, 481 (1994).

4 J. S. Koehler, Phys. Rev. B 2, 547 (1970).

5 S.I. Rao, P.M. Hazzledine, and D.M. Dimiduk, in Mat. Res. Soc. Symp. Proc. (Mat. Res. Soc., Pittsburgh, 1995), Vol. 362, pp. 67.

6E.O. Hall, Proc. Phys. Soc. B 64, 747 (1951).

7 N.J. Petch, Journal of the Iron and Steel Institute 173, 25 (1953).

${ }^{8}$ S. Suri, T. Neeraj, G.S. Daehn, D.H. Hou, J.M. Scott, R.W. Hayes, and M.J. Mills, Materials Science \& Engineering A 234-236, 996 (1997).

9 A.H. Cottrell, Prog. Met. Phys. 1, 105 (1949).

10 J.P. Hirth and J. Lothe, Theory of Dislocations, 2nd ed. (John Wiley \& Sons, New York, 1982).

11 A.H. Cottrell, Dislocations and Plastic Flow in Crystals (Clarendon Press, London, 1953).

12J.D. Eshelby, Philosophical Magazine 40, 903 (1949).

13 G. Leibfried, Zeitschrift für Physik 130, 214 (1951).

14P.M. Anderson and C. Li, NanoStructured Materials 5, 349 (1995).

15L.H. Friedman and D.C. Chrzan, Philosophical Magazine A 77, 1185 (1998).

16D.M. Barnett, Acta Metallurgica 15, 589 (1967).

17 The $I$, for "image", appears in the subscript because the self stresses are often referred to as image stresses. In some situations, they are found using the method of images $[10,18]$. .

18 Note that the argument of $g$ depends implicitly on $x c$ through $\sigma I$.

19J.O. Kim, J.D. Achenbach, P. B. Mirkarimi, M. Shinn, and S. A. Barnett, Journal of Applied Physics 72, 1805 (1992). 


\section{Continuum analysis of dislocation pileups - influence of sources}

\subsection{Overview}

Our studies this past year have focused on the role that sources play in the formation of pileups. Early in the year, it was determined that the problem of sources had not been treated adequately within the context of a grain size effect in a pure material. Since this is the logical starting point for further theory development, we began by including source information into the continuum theory of pileup formation. This problem, which we solved exactly, gives an expression for the strength of a material with a given grain size. To investigate the accuracy of the continuum approximation, we compare the results of the continuum theory with exact numerical results for a discrete dislocation model. The agreement between the continuum theory and the discrete dislocation model is excellent, implying that the continuum theory is a good description of pileup formation in systems with more than a few dislocations.

One explanation of the Hall-Petch relationship is that dislocation pileups serve to enhance the stress felt at grain boundaries. This dislocation pileup model results in a $d^{-1 / 2}$ dependence of the yield strength, with $d$ the grain size. In fact, this dependence is observed often. However, the traditional pileup picture neglects two important aspects of pileup formation: 1) the existence of a threshold stress for dislocation production, and 2) the necessity of a finite-sized, dislocation free region in which a source may operate. In this paper, both of these aspects are addressed within a continuum model of the dislocation pileups. A closed form expression is obtained for the dependence of yield stress on grain size and source characteristics. The continuum model agrees closely with the corresponding discrete dislocation model even when the pileup contains as few as ten dislocations.

\subsection{INTRODUCTION}

The Hall-Petch relation relates the yield stress, $\sigma^{*}$, of a polygranular material to its grain size, $d$

$$
\sigma^{*}=K d^{-1 / 2}+\sigma_{0}
$$

where $K$ and $\sigma_{0}$ are constants (Hall 1951, Petch 1953). There are multiple explanations for this relation (Hirth 1972, Armstrong 1970), with stress multiplication due to dislocation pileups as one of the most prominent and the earliest of the theories put forth (Hall 1951, Petch 1953, Hirth 1972).

The $d^{\mathrm{J} / 2}$ dependence of yield stress is predicted by the continuum theories of both the singleended and double-ended pileups. Furthermore, pileup formation against grain boundaries in the pre-macro strain region is observed commonly (Strong 1970). These observations suggest that continuum models of pileups can be considered successful.

Despite the apparent success of the continuum models, there are non-empirical intuitive reasons to modify them. Chief among these reasons is that the continuum models fail to take into account dislocation source characteristics, specifically, the critical source activation stress, the source position, and the lateral extent of the source in the slip plane. Fortunately, the continuum models can be modified to incorporate source characteristics. In so doing, the $d^{1 / 2}$ dependence in the Hall-Petch relation is not necessarily preserved. However, one important feature of the continuum model is preserved: One can derive an analytic function relating the yield stress to grain size and other physical parameters.

In this paper, the standard continuum theory of dislocation pileups is modified to include the aforementioned source characteristics. The effects of a friction stress are also discussed. (The new model will be referred to as the modified continuum theory, and earlier continuum theories will be referred to as standard continuum theories or source independent theories.) It is shown that the modified theory represents a more intuitive picture of pileups in equilibrium than the standard continuum theories. The modification to dislocation densities and the consequent 
modification to the Hall-Petch relation are analyzed and compared with discrete numerical calculations.

It is demonstrated that the modified theory predicts three regimes of behavior under the assumption that the source properties are independent of grain size. In the ultra-small grain size limit, where the grain size is less than about six times the lateral extent of the source, the yield strength rises rapidly, much faster than the $d^{1 / 2}$ power predicted by the Hall-Petch relation. For intermediate grain sizes, one recovers the standard $d^{1 / 2}$ dependence. In the limit of large grain sizes, the yield strength asymptotically approaches the source activations stress with a $d^{j}$ dependence, not $d^{1 / 2}$. These predictions closely approximate the predictions of a numerical model of discrete dislocations, particularly when the number of dislocation pairs is large ( $N \geq$ 10).

The remainder of this paper is organized as follows. In section 7.3, the physics of pileup formation is discussed. Section 7.4 presents the derivation of the source modified Hall-Petch relation and the approximations used. Section 7.5 presents a discussion. Section 7.6 presents a comparison of the continuum results with a discrete model. The conclusions are, presented in section 7.7 .

\subsection{PHYSICS OF PILEUP FORMATION}

Consideration of pileup formation in a simple, quasi-static picture suggests some new properties of a representative continuum dislocation density. The following analysis discusses the formation of double-ended pileups within a single grain, and the formation of single-ended pileups will be discussed briefly below.

It is imperative to define how source characteristics are introduced into the model. Mott's theory of source operation is used (Mott 1952), and it is assumed that source properties can be represented by two numbers, an activation stress, $\sigma_{c}$, and a position, $x_{0}$. One might object that a dislocation source might not have a well defined position if it has spatial extent in the direction of slip (e.g. a bowed Frank-Read source). $x_{0}$ is the "effective position" of the source, and it takes into account both the position and the spatial extent of the source. Rigorously, this means the source will operate if and only if the total stress at $x_{0}$ is greater than $\sigma_{c}$.

$$
\sigma\left(x_{o}\right)>\sigma_{c} \text {. }
$$

Figure 6-1 illustrates how a double-ended pileup forms from such a source. If, initially, the applied stress is less than the source activation stress, no dislocations are produced (not shown in figure). When the applied stress, $\sigma_{A}$, rises to just above $\sigma_{c}$, one dislocation dipole is produced (fig. 6-1(a)). (Dislocation dipoles are used in this model to represent loops formed by a FrankRead source.) The dipole expands under the action of the applied stress until it is blocked by grain boundaries or some other obstacle. The back stress from the dipole insures that the stress at the source now falls below $\sigma_{c}$, and no more dislocations are produced.

Increasing the applied stress compensates the back stress from the first dipole, and more dislocations are produced. The dislocations come to rest at a point determined by their mutual interaction and the applied stress. This process continues as the applied stress increases (see fig.

$6-1(b))$. Once the multiplied stress at the grain boundary reaches a critical value, $\tau_{b}^{*}$, yielding occurs. If the source parameters and the 


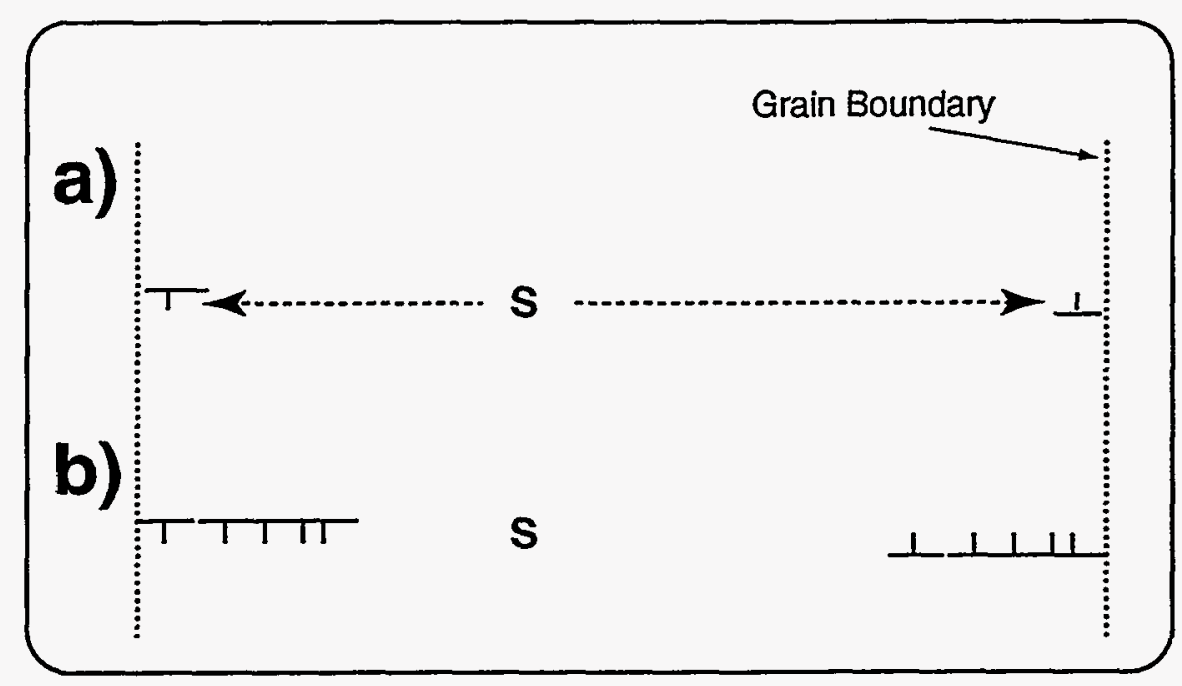

TRI-96342-6026-0

Fig. 6-1. SCHEMATIC DISLOCATION CONFIGURATIONS. The S represents a dislocation source. In panel (a), the applied stress, $\sigma_{A}$ is just above the critical source activation stress, $\sigma_{c}$. Only a single dislocation pair is produced because the resulting back stress inhibits further source operation. In panel (b), the applied stress, $\sigma_{A}$ is much greater than the source activation stress, $\sigma_{c}$. Initially, the additional applied stress overcomes the back stress from existing dislocations, and the source operates continuously. As the pileup builds, the back stress will eventually become great enough to inhibit source operation once again. For every value of $\sigma_{A}$ there will be an equilibrium number of dislocation dipoles.

grain boundary strength $\sigma_{b}^{*}$ are known, the yield stress can be predicted using the appropriate dislocation density.

A model similar to the one described above was implemented in a non-rigorous fashion by Lehoczky (1978) to explain deviation from the Hall-Petch relation in Al-Cu multilayers. The above model was also studied numerically by Anderson and $\mathrm{Li}$ for pileups in both homogeneous and inhomogeneous elastic media (Anderson and Li 1995). Anderson and Li did not observe a $d^{1 / 2}$ dependence of the yield stress in their model. (The limited range of their study did not include the $d^{1 / 2}$ regime.) Finally, a large portion of the continuum calculation was performed by Leibfried (1951). He obtained expressions for the modified dislocation density and resulting stress field for the case $x_{0}=0$ (i.e. the center of the double-ended pileup). However, Leibfried did not consider how the modified density affects the stress multiplication at the grain boundaries and consequently how it affects the yield stress prediction. An alternate derivation of the density profile is given in this paper.

There are three important properties of the pileup model which need to be noted and reflected in the continuum theory. The first is that yielding occurs when criteria local to the grain boundary are met through stress multiplication. This property is shared by both the standard continuum theory and the modified continuum theory introduced below. 

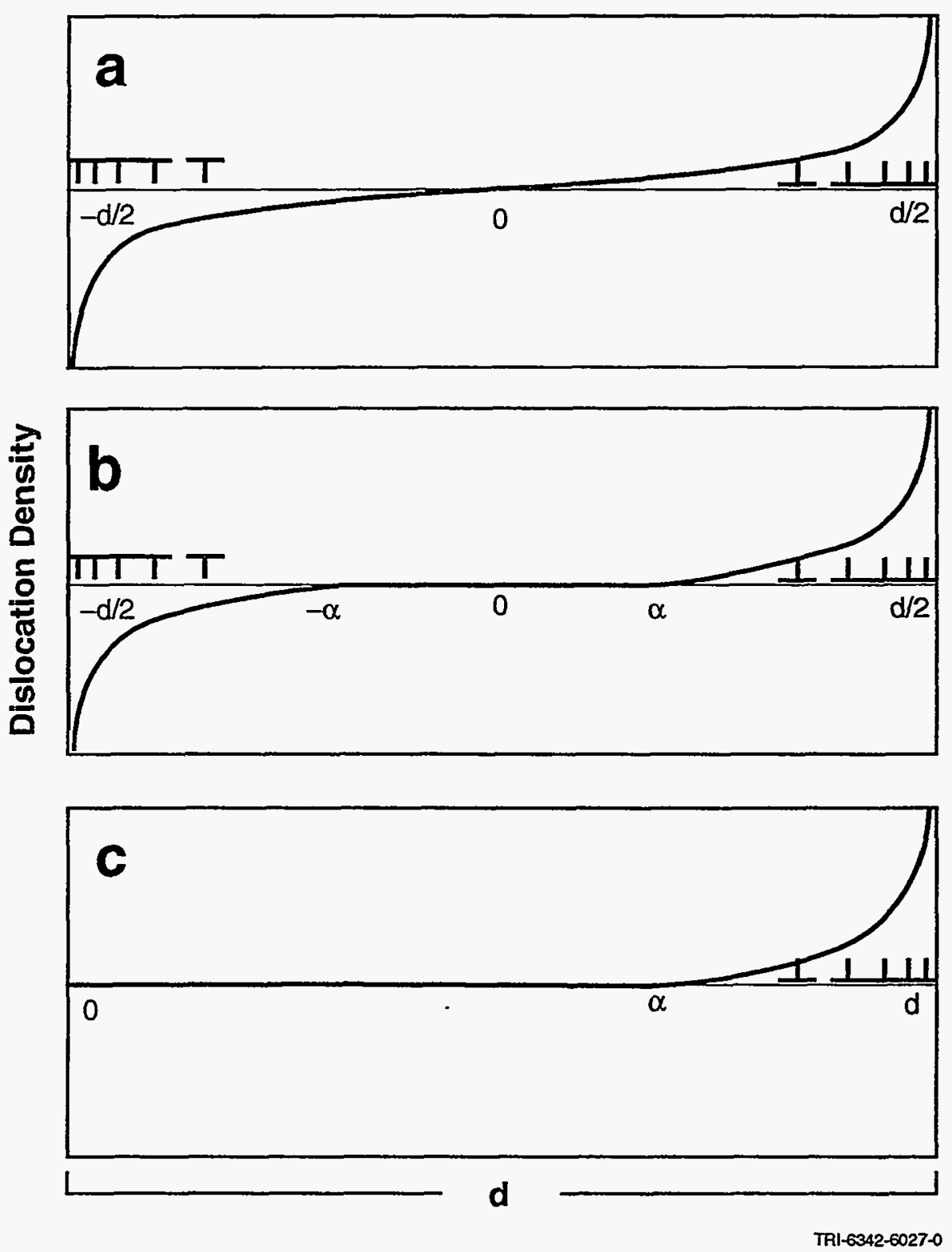

Fig. 6-2. (a) The dislocation density profile predicted by the source independent continuum theory for a double-ended pileup. (b) The density profile predicted by the modified continuum theory for a double-ended pileup. In the derivation of the density, the region containing the negative side of the pileup is referred to as $L_{1}=(d / 2, \alpha)$, and the positive region as $L_{2}=(\alpha$, $d / 2$ ). (c) The density profile predicted by the modified continuum theory for a single-ended pileup. The tail of the pileup terminates at $\alpha \in(0, d)$.

The next two properties are unique to the modified continuum theory. The second property is that the number of dislocations in the pileup is limited by back stress felt by the source. Consequently, the dislocation population $N$ is a function of the applied stress $\sigma_{A}$ and the source characteristics $\sigma_{c}$ and $x_{0}$. In the standard continuum theory $N$ is a function of $\sigma_{A}$ only. The third property is that the length of either half of the pileup increases with $\sigma_{A}$. As more dislocations are introduced to the pileup, the tails of the two ends move inwards, approaching the source. Since the tails are separated by a dislocation free region, the double-ended pileup may be thought of as two interacting super-dislocations. These distinctions between the standard continuum theory and the modified theory are illustrated in fig. 6-2. 
Besides distinguishing the source modified theory from the standard theory, the last two properties give rise to an immediate, quantitative result. In the limit $d \rightarrow \infty$, the superdislocations at either end are far removed from the source, and the back stress at the source is negligible. Consequently, any number of dislocations can be produced at stresses which are marginally above $\sigma_{c}$, i.e. $\sigma_{A}=\sigma_{c}+\varepsilon$. Another consequence of the negligible back stress is that the virtual work principle of Cottrell (1949) can be invoked as if the two ends of the pileup were exactly like single-ended pileups. In this care, the multiplied stress is

$$
\sigma_{b}=N \sigma_{A}=N\left(\sigma_{c}+\varepsilon\right) \text {. }
$$

Approximating that $\sigma_{A}=\sigma_{c}$, the number of dislocations in the equilibrium configuration just prior to yield is

$$
N=\left[\sigma_{b}^{*} / \sigma_{c}\right] \text {. }
$$

This number is significant in that it places an upper limit on the dislocation population for all $d<$ $\infty$, and it provides an order of magnitude estimate for the number of dislocations one can expect to see in medium- and large-sized grains prior to yielding.

Before continuing, there is one final comment on the standard continuum theory which must be made. The dislocation density allows no possible location for the source (see fig. 6-2(a)). Dislocations fill the entire slip plane. This characteristic is especially disturbing if the source is not located in the center of the grain or has a finite width. Intuitively, the dislocations must sit to the sides of the source, positively signed dislocations to one side, negatively signed dislocations to the other.

The above discussion applies to single-ended pileups as well. If one simply covers up the left hand side of fig. 6-1, the appropriate quasi-static picture for single-ended pileup is produced. The resulting density profile is illustrated in fig. 6-2(c).

\subsection{SOURCE MODIFIED HALL-PETCH RELATION}

The modified Hall-Petch relation is derived using several simplifying assumptions also used by Eshelby (1949), Eshelby, Frank and Nabarro (1951), Hall (1951), Petch (1953), Anderson and $\mathrm{Li}$ (1995), and others: 1) The elastic medium is assumed to be linear and isotropic. 2) Dislocations are assumed to be infinitely long, straight, parallel to each other and in the same slip plane. 3) Discrete dislocations are represented by a continuum dislocation density. 4) Initially, no internal stresses or friction stresses are taken into account. 5) The stress on the pileup's lead dislocations is used as the sole criterion for yielding. ${ }^{3}$ 6) As explained in the previous section, the pileup is assumed to grow as long as Mott's criterion, eqn. (2), is satisfied. 7) It is weakly assumed that the model parameters do not vary from grain to grain. If this assumption is invalid, one will have to interpret the model as applying to a single pile up in a single grain only. The yield stress predictions for individual grains would have to be incorporated into a more sophisticated statistical model to get experimentally verifiable yield stress predictions.

The derivation of the single-ended pileup model is trivial relative to the derivation of the double-ended model because the formulae used in the single-ended model are the same as for the source independent model; however, there is a difference in how the various parameters enter. Because of its simplicity the single-ended derivation will be presented first.

${ }^{1}$ This has been common practice in previous literature (Hall 1951); (Petch 1953); (Anderson and $\mathrm{Li} \mathrm{1995),} \mathrm{and} \mathrm{it} \mathrm{is} \mathrm{appropriate} \mathrm{to} \mathrm{a} \mathrm{model} \mathrm{in} \mathrm{which} \mathrm{the} \mathrm{lead} \mathrm{dislocation} \mathrm{punches} \mathrm{through} \mathrm{the} \mathrm{grain}$ boundary. It should also be noted that depinning of a dislocation source in an adjacent grain is often cited as the yielding mechanism. Despite this more accepted alternative, the stress on the lead dislocation has been used uniformly in models as the yielding criterion. This trend is most likely due to the simple formula of Cottrell (1949), $\sigma_{b}=N \sigma_{A}$. This trend is continued in this paper for ease of comparison. However, if one wishes to use the stress in an adjacent grain as the yielding criterion, eqns. (9) and (21) facilitate this. 


\subsection{1 single-ended pileup}

Both the discrete (Eshelby et al. 1951) and continuum (Eshelby 1949) theories were worked out originally by Eshelby, and the derivation of Leibfried (1951) can be found in Hirth and Lothe (1982). The following derivation will mimic the source-independent derivation closely.

The equilibrium dislocation density is assumed to be of the type depicted in fig. 6-2(c). The source is located at the left boundary, $x=0$. The head of the pileup is at the right grain boundary, $x=d$, and $\alpha \in(0, d)$ is the point where the pileup terminates. The exact density is found by enforcing the equilibrium condition, that the Peach-Köhler force vanish throughout the pileup. This condition is equivalent to requiring that the appropriately resolved stress vanish, and it is represented by an integral equation. For screw dislocations, this integral equation is

$$
0=\frac{\mu b}{2 \pi} P . V \cdot \int_{a}^{d} \frac{n\left(x^{\prime}\right) d x^{\prime}}{x-x^{\prime}}+\sigma_{A} ; x \in(\alpha, d)
$$

where P.V. indicates the principal value of the integral, $\mu$ is the shear modulus, $n(x)$ is the dislocation density to be determined, and $\sigma_{A}$ is a constant appropriately resolved applied stress. The corresponding equation for edge dislocations is obtained by the substitution $\mu \rightarrow \mu /\left(1_{-} v\right)$.

Equation 5 can be solved (Leibfried 1951, Eshelby 1949, Hirth and Lothe 1982), and the resulting density is (Hirth and Lothe 1982)

$$
n(x)=\frac{2 \sigma_{A}}{\mu b} \frac{\sqrt{x-\alpha}}{d-x}
$$

where $x \in(\alpha, d)$.

Next, it is necessary to solve for the unknown $\alpha$. This is accomplished as suggested by Mott (1952) by requiring that the source is at the threshold stress for dislocation production, i.e.

$$
\sigma\left(x_{0}=0\right)=\sigma
$$

where $\sigma(x)$ is the total stress at position $x$. To find $\sigma(x)$, it is first necessary to find $\sigma_{p}(x)$, the stress at $x$ resulting from the dislocations. This is given by (Hirth and Lothe 1982).

$$
\sigma_{p}(x)=-\sigma_{A}\left\lfloor 1-\frac{\sqrt{\alpha-x}}{\sqrt{d-x}}\right\rfloor .
$$

Thus,

$$
\sigma^{(x)}=\sigma_{A}+\sigma_{0}(x)=\sigma_{A} \frac{\sqrt{\alpha-x}}{\sqrt{d-x}} .
$$

Plugging eqn. (9) into eqn. (7) and solving for $\alpha$,

$$
\alpha=\left(\sigma_{r}^{\prime} \sigma_{A}\right)^{2} d \text {. }
$$

With $\alpha$ known, the density is easily integrated to give the number of dislocations (Hirth and Lothe 1982).

$$
N=\int_{a}^{d} n(x) d x=\frac{\pi \sigma_{A}(d-\alpha)}{\mu b}
$$

The multiplied stress, $\sigma_{b}$ is solved in identical fashion to the source independent case. The virtual work argument of Cottrell is invoked (Cottrell 1949) to give (Hirth and Lothe 1982) 


$$
\sigma_{b}=N_{\sigma_{A}}=\frac{\pi(d-\alpha) \sigma_{A}^{2}}{\mu^{b}}
$$

Plugging in $\alpha$, eqn. (12) is solved for $\sigma_{A}$. The yield stress is defined by the constraint that $\sigma_{A}$ $=\sigma^{*}$ when $\sigma_{b}=\sigma_{b}^{*}$. The predicted yield stress from this model is therefore

$$
\sigma^{*}=\sqrt{\frac{\mu b \sigma_{b}^{*}}{\pi d}+\sigma_{c}^{2}} .
$$

This prediction differs from that of the standard single-ended model only by the incorporation of the source activation stress, $\sigma_{c}$. When $\sigma_{c} \rightarrow 0$, the standard $d^{-1 / 2}$ prediction results.

\subsection{2 double-ended pileup}

The derivation of the double-ended solution parallels that of the single-ended result. Again, the equilibrium density comes from the solution of an integral equation.

$$
0=\frac{\mu b}{2 \pi} P . V \cdot \int_{L_{1}, L_{2}} \frac{n\left(x^{\prime}\right) d x^{\prime}}{x-x^{\prime}}+\sigma_{A} ; x \in L_{1}, L_{2}
$$

$L_{1}=(-d / 2,-\alpha) L_{2}=(\alpha, d / 2)$ (see fig. 6-2(b)), and $\sigma_{A}$ is the applied stress. This type of integral equation has a general solution given by Mikhlin (1957). In this case,

$$
n(x)=\frac{1}{i \pi} \frac{\sqrt{x+d / 2} \sqrt{x-\alpha}}{\sqrt{x+\alpha} \sqrt{x-d / 2}} \int_{L_{1}, L_{2}} \frac{f\left(x^{\prime}\right) d x^{\prime}}{x^{\prime}-x}+\frac{A x+B}{\sqrt{x+d / 2} \sqrt{x+\alpha} \sqrt{x-d / 2} \sqrt{x-\alpha}}
$$

with

$$
f\left(x^{\prime}\right)=\frac{2 \sigma_{A}}{i \mu b} \frac{\sqrt{x+\alpha} \sqrt{t^{\prime}-d / 2}}{\sqrt{x+d / 2} \sqrt{t-\alpha}}
$$

The second term in eqn. (15) is the homogeneous solution to the integral equation. After performing the integral (see mathematical appendix), setting $A$ and $B$ to meet the boundary condition $n(\alpha)=n(-\alpha)=0, A=-2 \sigma_{A}(\alpha-d / 2) / i \mu b$ and $B=2 \sigma_{A} \alpha(\alpha-d / 2) / i \mu b$, one finds

$$
n(x)=\left\{\begin{array}{cl}
\frac{-2 \sigma}{i \mu b} \frac{\sqrt{x+\alpha} \sqrt{x-\alpha}}{\sqrt{x+d / 2} \sqrt{x-d / 2}} \alpha \leq|x| \leq d / 2 \\
0 & \text { otherwise }
\end{array} .\right.
$$

Careful substitution for $x$ reveals that $n(x)$ has the same sign as $\sigma_{A}$ when $x \in L_{2}$ and the opposite sign as $\sigma_{A}$ when $x \in L_{1}$, as expected. One should also note that in the limit $\alpha \rightarrow 0$,

$$
\lim _{\alpha \rightarrow 0} n(x)=\frac{2 \sigma_{A}}{\mu b} \frac{x}{\sqrt{(d / 2)^{2}-x^{2}}}
$$

which is the solution for the source-independent double-pileup density (Hirth and Lothe 1982). Finally, in the limit of large separation between oppositely signed pileups,

$$
\alpha \rightarrow d / 2 \text { and } n(x) \rightarrow \begin{cases}\frac{2 \sigma}{\mu b} \sqrt{\frac{x-\alpha}{d / 2-x}} & x \in L_{1} \\ \frac{-2 \sigma}{\mu b} \sqrt{\frac{-x-\alpha}{x+d / 2}} & x \in L_{2}\end{cases}
$$


which are both single pileup density functions (Hirth and Lothe 1982). As the pileup separation becomes large with respect to the pileup width, $\alpha \square(d / 2-\alpha)$, the two pileups behave as if they were not interacting.

With the equilibrium distribution of dislocations known, it is possible to calculate the stress due to their presence. enoted (p, this stress is (see mathematical appendix)

\section{EMBED Equation.3}

Of course, it is alread known that $\left(p(x)=\sigma_{A} ; x \in L_{1}, L_{2}\right.$ (as expressed in eqn. (14)). After adding the applied stress, it is found that the total stress is

$$
\sigma(x)=\sigma_{p}(x)+\sigma_{A}=\sigma_{A} \frac{\sqrt{x+\alpha} \sqrt{t-\alpha}}{\sqrt{x+d / 2} \sqrt{x-d / 2}}
$$

The unknown $\alpha$ is found using the same source considerations as before,

$$
\sigma_{c}=\sigma\left(x_{0}\right)=\sigma_{A} \sqrt{\frac{\alpha^{2}-x_{0}^{2}}{(d / 2)^{2}-x_{0}^{2}}}
$$

from which one obtains

$$
\alpha=\sqrt{x_{0}^{2}+\left(\sigma_{c} / \sigma_{A}\right)^{2}\left((d / 2)^{2}-x_{0}^{2}\right)}
$$

With $\alpha$ known, one can solve for $N$, the number of dislocation in half the pileup. This is given in terms of elliptic integrals (Leibfried 1951).

Figure 6-3 is a plot of $N$ vs $\alpha / d$ rescaled by the standard double-pileup result, $N=\sigma_{A} d / \mu b$.

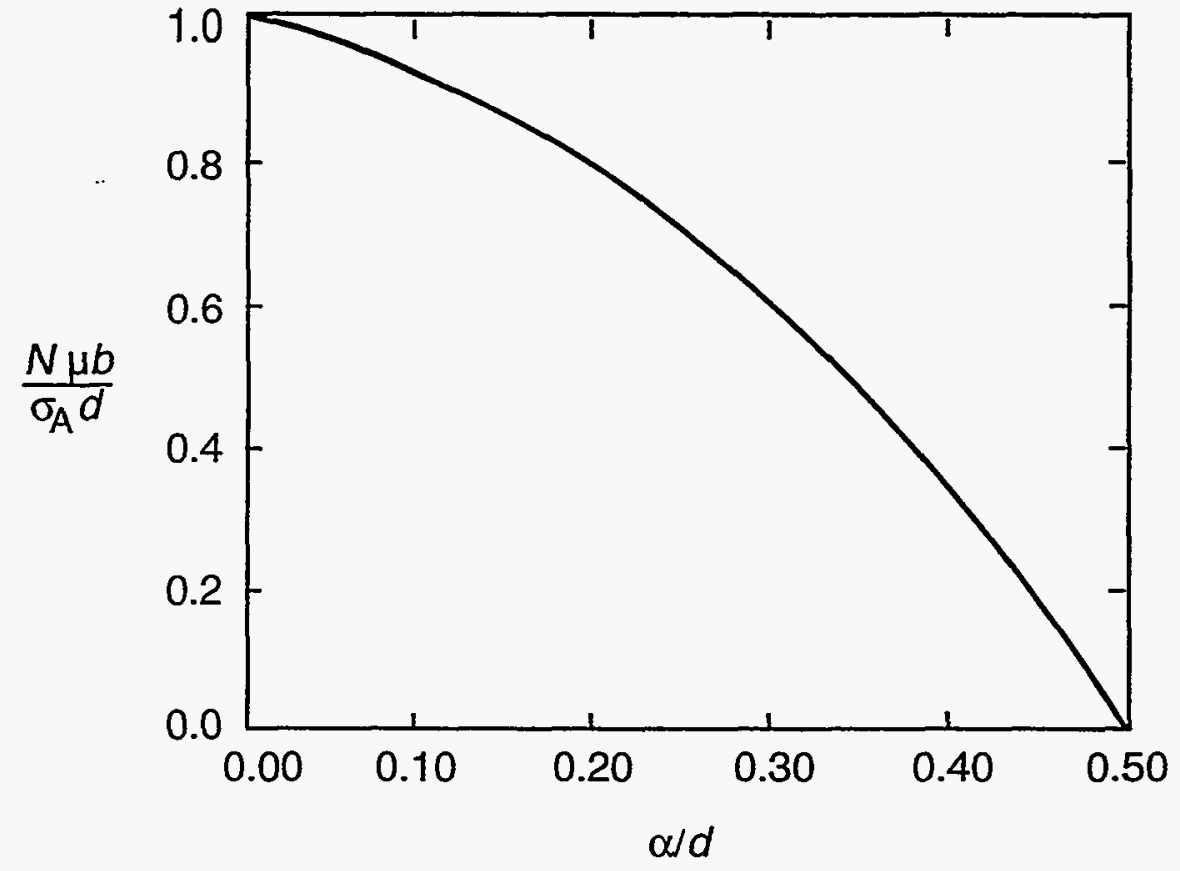

TRDI-6342-6029-0

Fig. 6-3. Number of dislocation dipoles predicted by the source modified continuum theory for a double-ended pileup (see eqn. (24)). Plot is normalized to the source independent continuum theory for which $N=\sigma_{A} d / \mu b$. 
The multiplied stress $\sigma_{b}$ at either end of the pileup is solved by considering the total external force on the positive half of the pileup. $\sigma_{b}$ for the negative half of the pileup is the same by symmetry.

$$
\alpha_{b}=\int_{L_{2}}\left(\sigma_{-}(x)+\sigma_{A}\right) n(x) d x
$$

where

$$
\sigma_{-}(x)=\frac{\mu b}{2 \pi} \int_{L_{2}} \frac{n\left(x^{\prime}\right) d x^{\prime}}{x-x^{\prime}}
$$

which is the stress resulting from the negative half of the pileup. In general, $\sigma_{-}(x)$ cannot be evaluated easily. However, the integral in eqn. (25) can be performed (see mathematical appendix), and $\sigma_{A}$ determined as a function of $\alpha$ and $\sigma_{b}$ :

$$
\sigma_{A}=\sqrt{\frac{2 \mu b \sigma_{b}}{\pi} \frac{d / 2}{(d / 2)^{2}-x_{0}^{2}}+\sigma_{c}^{2}}
$$

In the limit $x_{0} \rightarrow 0$ with finite $\sigma_{c}$,

$$
\sigma_{A}=\sqrt{\frac{4 \mu b \sigma_{b}}{\pi d}+\sigma_{c}^{2}}
$$

which is very similar to the single-ended pileup result (eqn. (13)).

Both eqns. (13) and (28) can be cast in the form

$$
\sigma^{*}=\sqrt{K_{H P}^{2} d^{-1}+\sigma_{c}^{2}}
$$

with $K_{H P}=\left(\mu b \sigma_{b}^{*} / \pi\right)^{1 / 2}$ for the single-ended case, and $K_{H P}=\left(4 \mu b \sigma_{b}^{*} / \pi\right)^{1 / 2}$ for the doubleended case.

\subsection{DISCUSSION}

In order to make experimental use of eqns. (27) - (29), one must know how $\sigma_{c}$, varies with grain size $d$. This variation may not be trivial. One experimental study of grain boundary sources was performed by Tangri and Tandon (1975). In this experiment, it was found that $\sigma_{c}$ also obeys the Hall-Petch relation (i.e. $\sigma_{c}=K d^{-1 / 2}+\sigma_{0}$ ). In the case of eqn. (27) where $x_{0}$ may not be zero, there is even more complication since $x_{0}$ may depend on grain size and vary from grain to grain. The $x_{0} \neq 0$ case introduces new small scale behavior; this discussion is deferred to section V, discrete model. In this section, it is assumed that $x_{0}=0$. Furthermore, rather than speculate on the dependence $\sigma_{c}$ might have on grain size or how it might vary from grain to grain, the simplest case $\sigma_{c}=$ constant will be considered here. These assumptions, chosen for convenience, allow one to see how including a source in the standard pileup model can alter the Hall-Petch relation.

\subsection{1 source-dependent length scale}

Equation (29), in which $x_{0} \rightarrow 0$, shows that the Hall-Petch dependence is influenced by a competition between the first grain size dependent term, and the second constant term. This competition gives rise to a new length scale. The terms are equal when

$$
d=d^{*} \equiv \frac{K_{H P}^{2}}{\sigma_{c}^{2}} .
$$

In the small $d$ limit, meaning $d \rightarrow d^{*}$, 


$$
\sigma^{*} \approx \frac{K_{H P}}{\sqrt{d}}+\frac{\sigma_{c}^{2}}{2 K_{H P}} \sqrt{d} .
$$

To lowest order, the source-independent Hall-Petch relation is obtained except for the constant offset. In the large $d$ limit,

$$
\sigma^{*} \approx \sigma_{c}+\frac{K_{H P}^{2}}{2 \sigma_{c}} \frac{1}{d} .
$$

The yield stress approaches a constant not as $d^{-1 / 2}$ but as $d^{-1}$. In the traditional regime, $d \sim d^{*}$, the exponent of $d$ varies smoothly from $11 / 2$ to $\_1$. Written in a more traditional way,

$$
\sigma^{*}=K_{H P} d^{-n}+\sigma_{0}
$$

where $n$ runs with $d$ from $1 / 2$ to 1 , and $K_{H P}$ changes accordingly (see fig. 6-4).

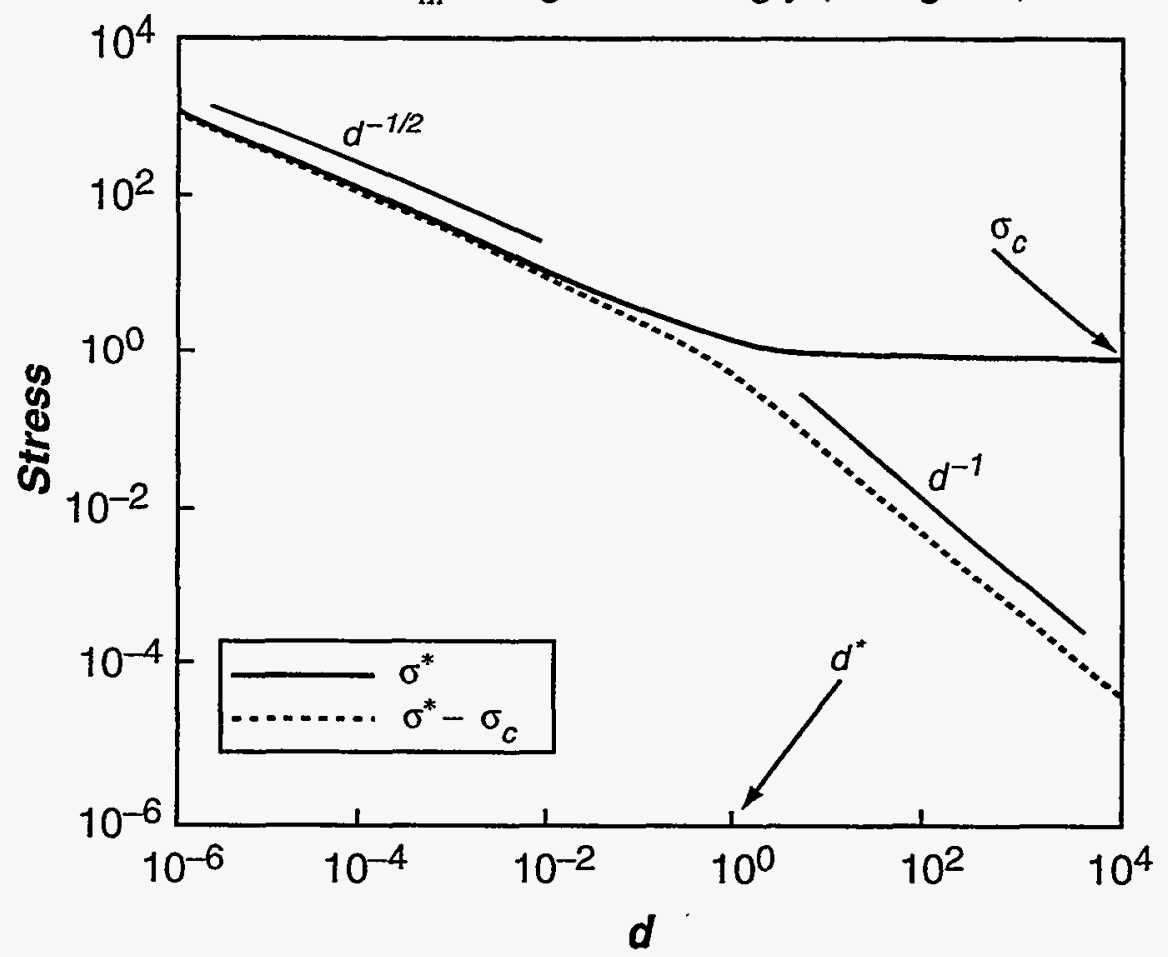

TRI- $6342-6030-0$

Fig. 6-4. plot of eqn, (29) with $K_{H P}=\sigma_{c}=1$.

Since $\sigma_{c}$ is generally not known, $d^{*}$ itself is not known. Furthermore, $d^{*}$ is only well-defined if $\sigma_{c}$ is independent of grain size. Bearing these cautionary notes in mind, $d^{*}$ can be estimated. A suggested value of $\sigma_{c}$ for dislocation sources in grain boundaries is $\mu / 1000$ (Vaxin et al. 1987). Then, using HallPetch slopes, $K_{H P}$, given by Armstrong (1970), one finds, using formula 30 , that for Mo, $d \approx 206 \mu \mathrm{m}$, and f $0 \mathrm{r}$

\subsection{2 friction}

The calculations up until now have not included friction. Inclusion of friction, however, requires only a slight modification to the formalism. Such a modification to Eshelby's theory of single-ended pileups (Eshelby 1949) was made by Petch (1953). The friction stress $\sigma_{0}$ is defined 
as the stress below which a dislocation's velocity is zero. The effect of friction can be accounted for easily by the substitution

$$
\sigma_{A} \rightarrow \sigma_{A}-\sigma_{0}
$$

wherever $\sigma_{A}$ appears in the friction-free formulae and by reinterpreting the parameters, $\sigma_{c}$ and $\sigma_{b}^{*}$.

The substitution can be seen to be both necessary and sufficient by reconsidering the equilibrium condition expressed in eqns. (5) and (22). Instead of requiring that the stress felt by all dislocations vanish, it is required that the stress felt be $\sigma_{0}$. This condition is not suitable if one wishes to consider cyclic loading, but it is appropriate if $\sigma_{A}$ is increased monotonically. The corresponding equilibrium condition is expressed by

$$
0=\frac{\mu b}{2 \pi} P . V \cdot \int \frac{n\left(x^{\prime}\right) d x^{\prime}}{x-x^{\prime}}+\left(\sigma_{A}-\sigma_{0}\right)
$$

Reinterpreting $\sigma_{c}$ and $\sigma_{b}^{*}$ is equally simple. Instead of the source activation stress, $\sigma_{c}$ represents the amount of stress in addition to $\sigma_{0}$, which is required to activate a source. In the cast of a Frank-Read source where one would have to overcome both the friction $\sigma_{0}$ and line tension $\sigma_{T}$ of the source, the activation stress is

$$
\Sigma_{c}=\sigma_{0}+\sigma_{T}
$$

In this case, $\sigma_{c} \equiv \sigma_{T}$ should be used. Similarly,

$$
\sigma_{b}^{*}=\Sigma_{b}^{*}-\sigma_{0}
$$

where $\Sigma_{b}^{*}$ is the critical boundary stress. Unlike $\sigma_{c}$, there is no physical interpretation of $\tau_{b}^{*}$.

Interpreting $\sigma_{c}$ and $\sigma_{b}^{*}$ as above, the source modified Hall-Petch relation becomes for singleended pileups,

$$
\sigma^{*}=\sqrt{\frac{\mu b \sigma_{b}^{*}}{\pi d}+\sigma_{c}^{2}}+\sigma_{0}
$$

and for double-ended pileups,

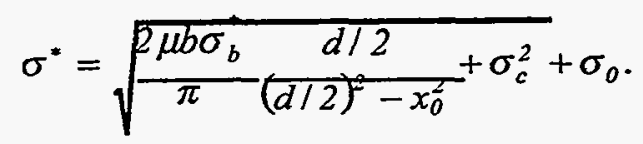

In the limit, $d \rightarrow d^{*}$, eqn. (38) becomes identical to eqn. (1), and with the additional constraint, $x_{0}$ $\rightarrow 0$, so does eqn. (39).

\subsection{DISCRETE MODEL}

\subsection{1 description}

The predictions of the modified continuum theory of double-ended pileups are compared with those of a numerical model of discrete dislocation dipoles. The discrete model is similar to the continuum model with a few important differences. First, because dislocations are produced discretely, it is not always possible to satisfy eqn. (22), which requires that the stress, at the - source be just below the stress required for dislocation production. Also, it is sometimes possible that yielding will occur when $\sigma_{b}$, the stress on the lead dislocation, is greater $\operatorname{than} \sigma_{b}^{*}$, the critical lead stress. This might happen when the source injects new dislocations into the 
pileup. In the discrete case, the yield stress is found from the following criteria: The smallest applied stress, $\sigma_{A}$, is found such that

$$
\sigma\left(x_{0}\right) \leq \sigma_{c}
$$

and

$$
\sigma_{b} \geq \sigma_{b}^{*}
$$

The configurations are found by the same physical requirement used in the continuum model, that the total stress felt by each dislocation vanish.

Another important difference between the continuum and discrete models is that it is impossible to set $x_{0}=0$ in the discrete case. This restriction comes from the attraction of the newly produced dislocation to its oppositely signed partner. This restriction was noted by Anderson and $\mathrm{Li}(1995)$, who set $\sigma_{c}$, to be the stress required to pull apart an incipient dipole. This assignment of $\sigma_{c}$ is not absolutely necessary, but it does place a lower bound on $\sigma_{c}$. For screw dislocations,

$$
\sigma_{c} \geq \frac{\mu b}{2 \pi} \frac{1}{2 x_{0}} .
$$

This incipient dipole problem also arose in the numerical calculations of double-ended pileup configurations by Armstrong, Chou, Fisher and Louat (1966). They did not consider the effects of sources, but instead noted the existence of two equilibrium configurations, one stable and one unstable.

At first, this restriction may seem an artifact of approximating dislocation loops by two infinite straight dislocations. For one thing, as $x_{0} \rightarrow 0, \sigma_{c} \rightarrow \infty$. However, this inverse correlation between $x_{0}$ and $\sigma_{c}$ is reminiscent of Frank-Read sources. If one uses the activation stress formula given by Hirth and Lothe (1982),

$$
\sigma_{c} \approx \frac{\mu b}{L}
$$

where $L$ is the length of the pinned segment which comprises the Frank-Read source, one finds that eqn. (42) is not very restrictive. This can be seen by considering that the Frank-Read source must bow out to a distance of order $L / 2$ before it can operate. Setting $x_{0}=L / 2$, eqn. (42) places a lower limit on $\sigma_{c}$

\subsection{2 analysis and comparison with continuum results}

In general, the discrete and continuum models give predictions, which agree rather closely, but not perfectly. Specifically, the modified continuum model tends to overestimate the yield strength, and it does not always produce the correct configurational predictions. However, the differences are small, particularly for large numbers of dislocations. Furthermore, both models predict that as the grain size approaches $2 x_{0}$, the yield stress rises more rapidly than the _1/2 power law predicted by the Hall-Petch relation.

The strength predictions, $\sigma^{*}$ agree well over most of the grain size range, but depart somewhat for the smaller grain sizes. These predictions are shown in figs. 6-5 and 6-6 for the following parameters: $\mu=1, \sigma_{c}=0.003 \mu, b=3 \AA, x_{0}=0.01 \mu \mathrm{m}$ (slightly above the minimum value set by eqn. (42), $\left.7.96 \_10^{-3} \mu \mathrm{m}\right), \sigma_{b}^{*}=25 \sigma_{c}, 50 \sigma_{c}$, and $100 \sigma_{c}$.

A most notable feature of the curves in figs. 6-5 and 6-6 is that $\sigma^{*}$ starts to diverge as $d \rightarrow 2 x_{0}$ for both the continuum and discrete model. In the continuum case, $\sigma^{*}$ diverges for $d=2 x_{0}$ as $1 / \sqrt{d-2 x_{0}}$. This divergence is derived easily from eqn. (27). In figs. 6-5(a) and 6$6(\mathrm{a}), N$, the number of dipoles in the pileup becomes very small. Obviously, the continuum model is less trustworthy in this limit. One might be 

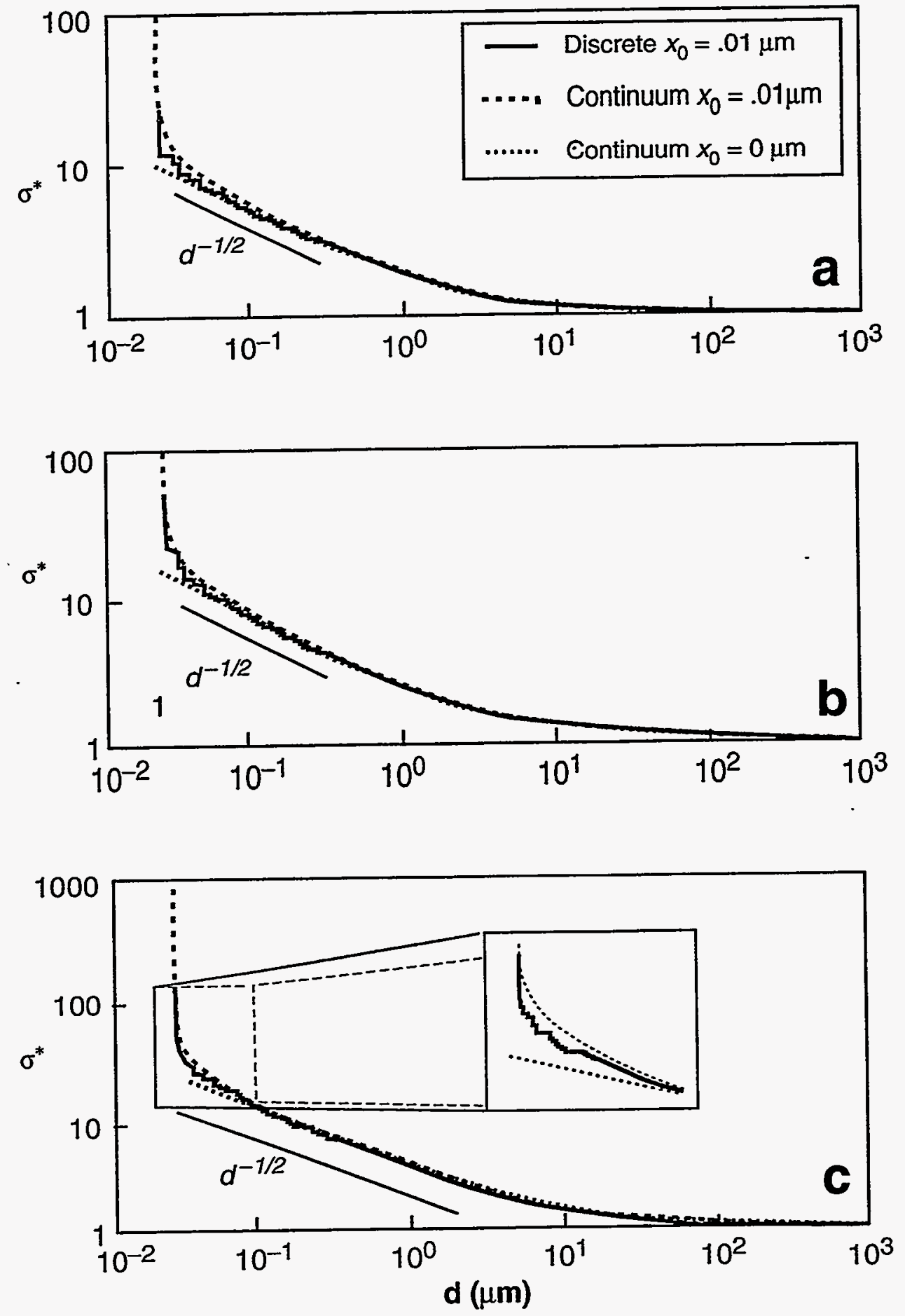

TRI-\$342-6031-0

Fig. 6-5 Log-log plots of predicted yield stress vs. grain size from the discrete and continuum models of double ended pileups. In all plots, $\mu=1, \sigma_{c}=0.003 \mu, b=3 \AA$. The value of $\tau_{b}^{*}$ varies from panel to panel. In (a), $\sigma_{b}^{*}=25 \sigma_{c}$, and $d^{*}=79.6 \mu \mathrm{m}$. In (b) $\sigma_{b}^{*}=50 \sigma_{c}$, and $d^{*}=318.3 \mu \mathrm{m}$. In (c) $\sigma_{b}^{*}=100 \sigma_{c}$, and $d^{*}=1273.2 \mu \mathrm{m}$. 

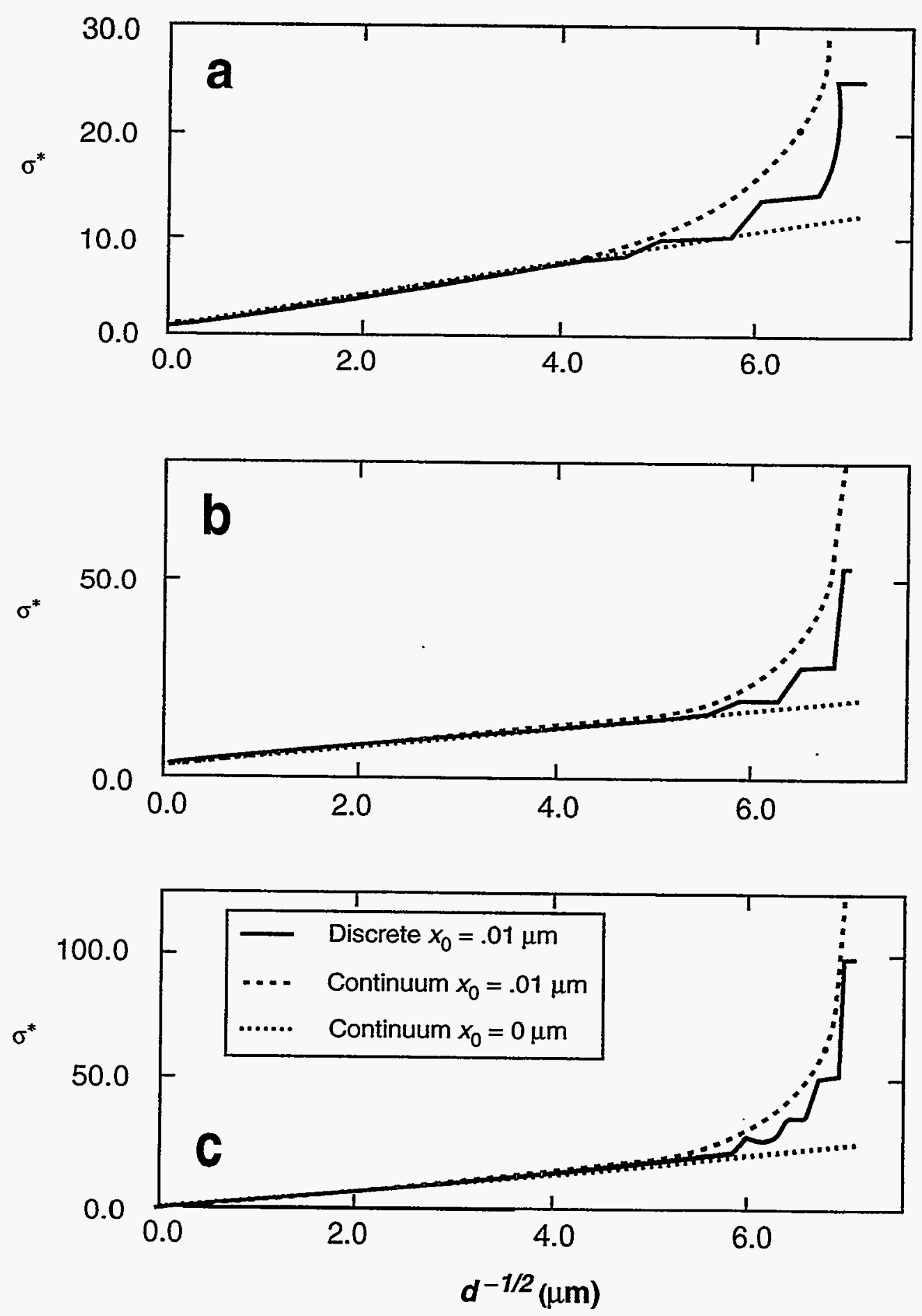

TRI-6342-6033-0

Fig. 6-6 Predicted yield stress vs. $d^{1 / 2}$ from the discrete and continuum models of double-ended pileups. In all plots, $\mu=1, \sigma_{c}=0.003 \mu, b=3 \AA$. The value of $\sigma_{b}^{*}$ varies from panel to panel. In (a), $\sigma_{b}^{*}=25 \sigma_{c}$, and $d^{*-1 / 2}=.112 \mu \mathrm{m}^{-1 / 2}$. In (b), $\sigma_{b}^{*}=50 \sigma_{c}$, and $d^{*-1 / 2}=5.61 \times 10^{2} \mu \mathrm{m}^{-1 / 2}$. In (c) $\sigma_{b}^{*}=100 \sigma_{c}$, and $d^{*-1 / 2}=2.80 \times 10^{-2} \mu \mathrm{m}^{-1 / 2}$. 
tempted to remove the continuum divergence by setting $x_{0} \equiv 0$ and using eqn. (28). If one does this, one finds that eqn. (28) tends to underestimate $\sigma^{*}$ more than eqn. (27) tends to overestimate (see figs. 6-5 and 6-6). One could, alternatively, use both equations to produce approximate lower and upper bounds. In figs.6-5(c) and 6-6(c), agreement in the small regime is better. Finally, the predictions of both the continuum and discrete model must be called into question for the ultra-small regime because the models assume that dislocations are infinite and straight. This assumption is less valid as the size of the pileup decreases. However, a pileup model of circular dislocations was shown to be similar to the more traditional straight dislocation models for the source independent case ( $\mathrm{Li}$ and $\mathrm{Liu}$ 1967). Perhaps a similar result applies here.

In addition to the yield stress predictions, the continuum and discrete models also give configurational predictions. The configurational predictions of the continuum model are not too different from the discrete predictions in so much as it is possible to compare them. Two methods are used to test the accuracy of the continuum computational predictions. First, $N$, the number of dipoles for the configurations at the yield stress are compared. Second, the density profiles are compared. These comparisons must be made using a single set of parameter values. Unfortunately, these sets are non-unique. One can choose the set $\left(\sigma_{A}, \sigma_{c}, d\right)$ or $\left(\sigma_{c}, \sigma_{b}, d\right)$, etc. This choice is complicated by the fact that the transformations from one set to another are different depending on whether one is discussing the -continuum or the discrete model. In the following discussion, the configurations are compared for a given parameter set, $\left(\sigma_{c}, \sigma_{b}, d\right)$

Because $\sigma_{A}$ is not one of the chosen parameters, in some cases the configurations will be compared at different applied stresses $\sigma_{A}$.

The predictions for $N$ are given using the same parameter values as were used for the yield stress predictions (see fig. 6-7). The $N$ predictions are in better agreement than the yield stress predictions (figs. 6-5 and 6-6). Also, the continuum model, as a rule, has slightly fewer dislocations than the discrete model. So far, in all cases, this difference has been fractional, so it is only important for small $N$. The difference also helps to explain why the continuum model predicts higher yield as than the discrete model.

The predictions for the density profiles are also in good agreement, at least for large $N$ (as one would expect). In the following discussion, the density profiles will not be compared directly. Instead, their integrated weights will be compared. The necessity for this indirect analysis is brought about by the roughness of the discrete density. To compare the discrete with the continuum model, it is noted that the discrete model's dislocation density function can be represented by

$$
n(x)=\sum_{i=1}^{2 N}( \pm)_{i} \delta\left(x-x_{i}\right)
$$




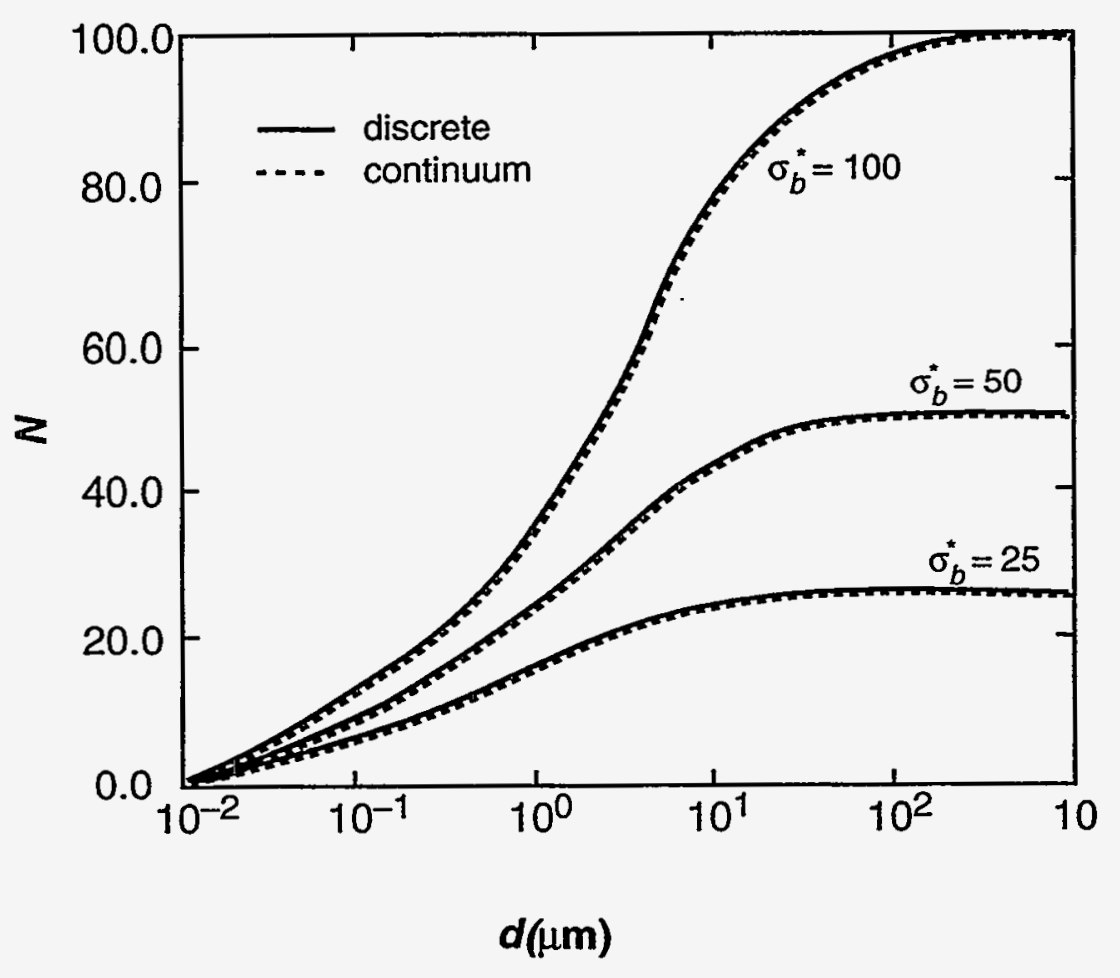

TRI-6342-6032-0

Fig. 6-7. Predicted number of dislocation dipoles, $N$ vs. grain size from the discrete and continuum models of double-ended pileups. In all three plots, $\mu=1, \sigma_{c}=0.003 \mu, b=3 \AA$ and $x_{0}$ $=10^{-2} \mu \mathrm{m}$. $\sigma_{b}^{*}=25 \sigma_{c}, 50 \sigma_{c}$, and $100 \sigma_{c}$. Agreement is very good, so it is difficult to distinguish the curves.

where $\left\{x_{i}\right\}$ are the equilibrium dislocation positions, $( \pm)_{i}$ indicates the sign of the $i^{\text {th }}$ dislocation, and $\delta$ is the Dirac delta distribution. The densities, eqn. (44) and 17 are integrated to give $N(x)$. Taking the center of the grain to be the origin,

$$
N(x)=\int_{0}^{x} n\left(x^{\prime}\right) d x^{\prime}
$$

The $N(x)$ 's are shown for three different sets of parameters in fig. 6-8. As expected, agreement gets better for pileups with large numbers of dislocations. However, agreement is not too bad even when $N$ is as small as 10 (see fig. 6-8(b)).

\subsection{CONCLUSIONS}

In this paper, the standard dislocation-pileup based theory of the Hall-Petch relation has been extended to include dislocation source characteristics, a critical stress for source activation, and a source position. The influence of a friction stress has also been incorporated. The modified theory differs from the source independent theory chiefly by the existence of a finite dislocationfree region in which the source operates. A closed-form expression for the dependence of yield strength on grain size and source parameters has been presented and analyzed.

Specifically, it is demonstrated that the introduction of a rudimentary source characterized by $x_{0}$ and $\sigma_{c}$, can alter the yield stress predictions of the Hall-Petch relation. Traditionally, pileup models have taken the grain boundary strength, $\sigma_{b}^{*}$ to be independent of grain size. In this study, the newly introduced parameters, $x_{0}$ and $\sigma_{c}$ are also assumed to be independent of grain size. Using these assumptions, three regimes are identified. The smallest regime occurs when $d \rightarrow$ $2 x_{0}$. In this regime, the yield stress starts to diverge. In the intermediate regime in which $x_{0} \rightarrow d$ 
$\rightarrow d^{*}$, with $d^{*}=K_{H P}^{2} / \sigma_{c}^{2}$ the predicted behavior is identical to that predicted by the source independent Hall-Petch relation. For $d \square d^{*}$, the yield strength approaches a constant with a $1 / d$ dependence. It is also noted that within the current assumption, the introduction of the source activation stress does not explain the constant $\sigma_{0}$ term within the Hall-Petch relation. As in previous pileup models, explanation of the constant term requires the inclusion of a friction stress.

Comparison of the continuum model with a discrete model indicates that the continuum model is accurate for large $d$, but becomes less accurate for small $d$. Empirically, it is observed that the modified Hall-Petch relation, with $x_{0} \neq 0$, overestimates the yield strength for small $d$. Setting $x_{0}=0$ in the modified Hall-Petch relation gives an approximate lower bound on the yield strength predicted by the discrete model. For all length scales, including small $d$, qualitative and nearly quantitative agreement is obtained.

A final note which is pertinent to all of the previous discussion is that no account was taken of how the model parameters including grain size, $d$, might fluctuate across a sample. A more complete understanding of these fluctuations as well as an understanding of the properties of dislocation sources operating within a strained sample, may lead to a more complete understanding of yielding in polygranular materials. 

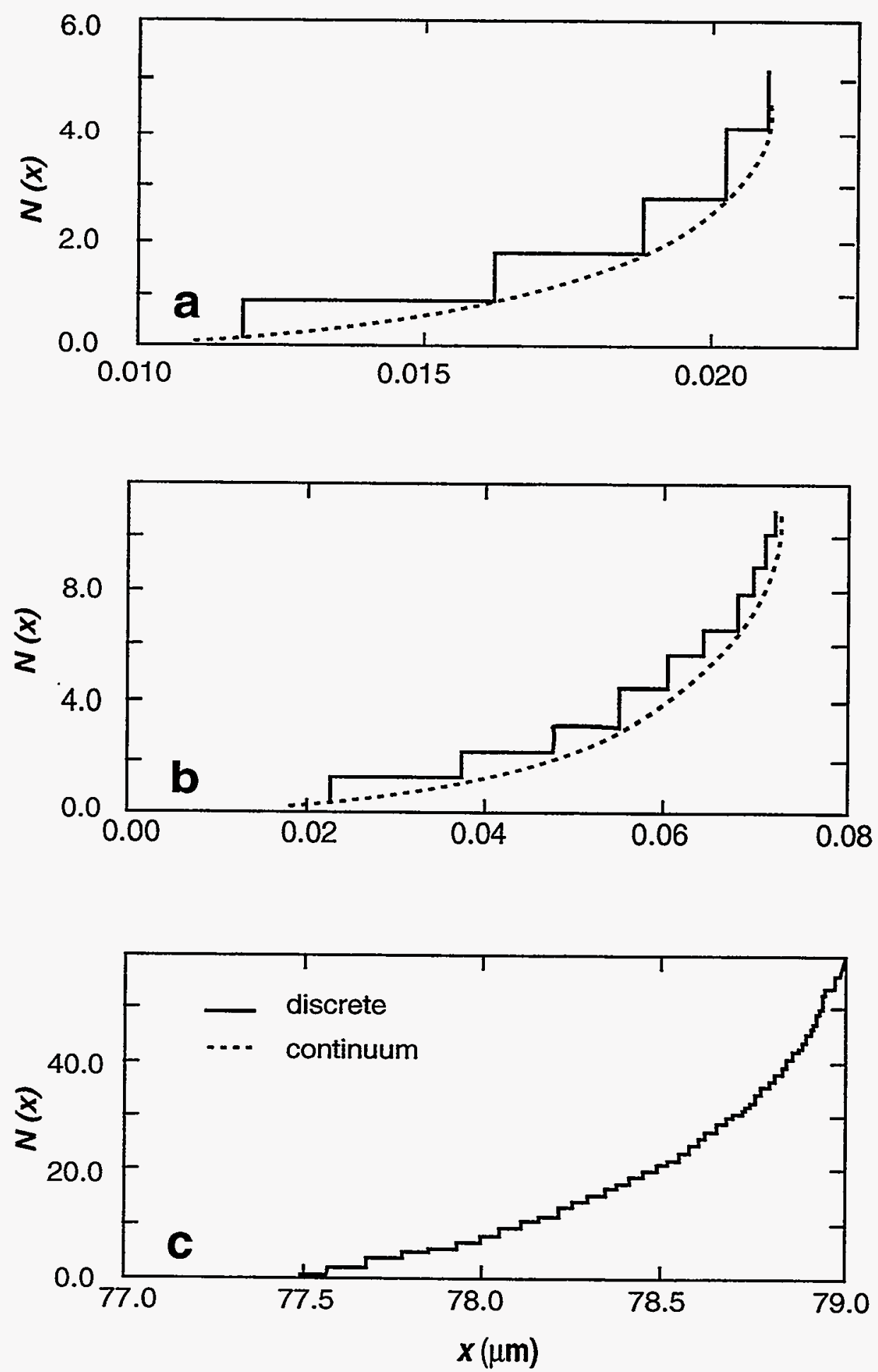

Fig. 6-8. Integrated density, $N(x)$ vs. $x$ (see eqn. (45)). $N(x)$ is shown in each case only in the domain $(\alpha, d / 2) . \mu=1, \sigma_{c}=0.003 \mu, b=3 \AA$ and $\sigma_{b}^{*}=50 \sigma_{c^{\circ}}$ In (a), $d=0.0417 \mu \mathrm{m}$, and $N=5$. In (b) $d=.1432 \mu \mathrm{m}$, and $N=10$. In (c) $d=157.9 \mu \mathrm{m}$, and $N=50$. 


\subsection{ACKNOWLEDGMENTS}

The authors thank K. Lin and A. K. Faradjian for useful discussions. The authors gratefully acknowledge Sandia National Laboratories for supporting this research.

\subsection{REFERENCES}

ANDERson, P. M. AND LI, C., 1995, NanoStructured Materials, 5, 349.

ARMSTRONG, R. W., 1970, Advances in Materials Research, 4, 101.

ARMSTRONG, R. W., ChOU, Y. T., FISHER, R. M., AND LouAT, N., 1966, Philosophical Magazine, 14, 943.

Cottrell, A. H., 1949, Prog. Met. Phys., 1, 105.

ESHELBY, J. D., 1949, Philosophical Magazine, 40, 903.

ESHELBy, J. D., FRANK, F. C., AND NABARRO, F. R. N., 1951, Philosophical Magazine, $42,351$.

Hall, E. O., 1951, Proc. Phys. Soc. B. 64, 747.

HIRTH, J. P., 1972, Metallurgical Transactions, 3, 3047.

HIRTH, J. P. AND LOTHE, J., 1982, Theory of Dislocations. John Wiley \& Sons, New York, second edition.

LEHOCZKY, S. L., 1978, Phys. Rev. Let, 41, 1814.

LEIBFRIED, G., 1951, Zeitschrift für Physick, 130, 214.

LI, J. C. M. AND LIU, G. T., 1967, Philosophical Magazine, 15, 1059.

MIKHLIN, S. G., 1957, Integral Equations and Their Applications to Certain Problems in Mechanics, Mathematical Physics and Technology, volume 4 of International Series of Monographs on Pure and Applied Mathematics. Pergamon Press, New York.

MorT, N. F., 1952, Philosophical Magazine, 43, 1151.

PETCH, N. J., 1953, Journal of the Iron and Steel Institute, 173, 25.

TANGRI, K. AND TANDON, K. N., 1975, In D. W. J. Walter, J. H. Westbrook, editor, Grain Boundaries in Engineering Materials, Proc. $4^{\text {th }}$ Bolton Landing Conf. 1974, page 327, Baton Rouge, LA. Claitor's Publishing Division.

VARIN, R., KURZYDLOWSKI, K. J., AND TANGRI, K., 1987, Materials Science and Engineering, 85, 115. 


\subsection{APPENDIX A: MATHEMATICAL APPENDIX}

\subsection{1 equation (15)}

The integral in eqn. (15) is performed by converting it to a contour integral in the complex plane. The analytical continuation of $f\left(x^{\prime}\right)$ (eqn. (16)), $f\left(z^{\prime}\right)$ has four branch points and two branch cuts in the complex plane (see fig. 6-9).
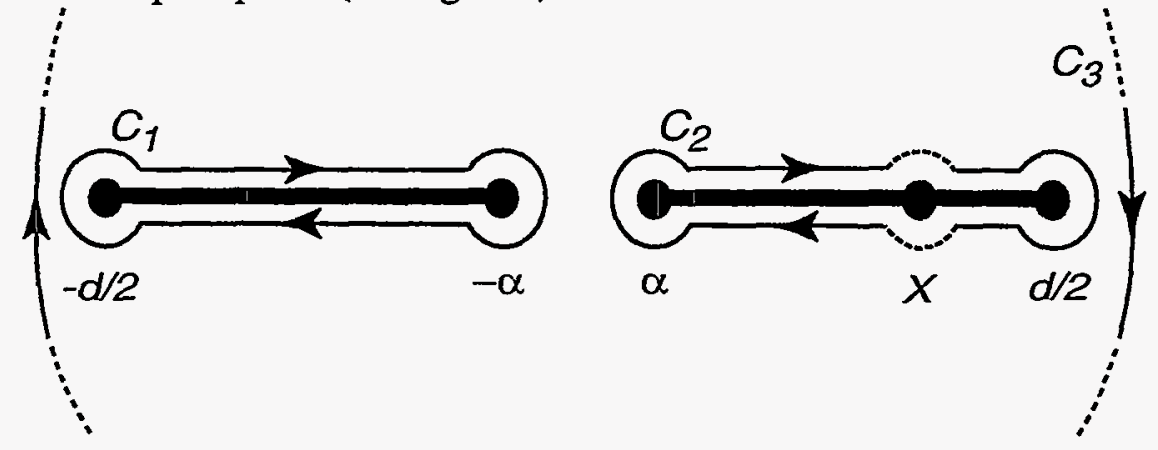

FIG. 6-9. $F\left(Z^{\prime}\right) /(Z-X)$ (SEE EQN. 16)) HAS TWO BRANCH CUTS, ONE FROM - D/2 TO - $\alpha$ AND ONE FROM $\alpha$ TO D/2. THERE IS ALSO A POLE AT $X$. INTEGRATING OVER THE CLOSED CONTOURS $C_{1}, C_{2}$ IS EQUIVALENT TO $2 \times$ THE INTEGRAL OVER THE OPEN CONTOURS $L_{1}=(-D / 2,-\alpha), L_{2}=(\alpha$, $D / 2)$. FINALLY, THE CONTOURS $C_{1}, C_{2}$ ARE DEFORMED INTO THE CONTOUR $C_{3}$, AN INFINITE CIRCLE.

Integrating over $L_{1}$ and $L_{2}$ is equivalent to integrating just above the branch cuts. If one continues integrating along a path around a branch point to the lower side of a cut, $f\left(z^{\prime}\right)$ and the integrand change sign. Since $d z^{\prime}$ also changes sign after going around a branch point, the integral over $L_{1}, L_{2}$ is equal to half the integral over $C_{1}, C_{2}$. There is, however, one additional complication. Since $x$ must lie on $L_{1}$ or $L_{2}$ one of the contours $C_{1}, C_{2}$ is not closed due to the singularity at $x$. This contour may be closed by adding in the appropriate half-loops above and below the branch cut at the singular point (fig. 6-9). The upper half-loop contributes exactly $i \pi f(x)$, and the lower half-loop contributes exactly $i \pi \lim _{\varepsilon \rightarrow 0} f(x-i \varepsilon)$. Since $x-i \varepsilon$ is just opposite $\mathrm{x}$ across the branch cut; $\lim _{\varepsilon \rightarrow 0} f(x-i \varepsilon)=-f(x)$, and the total contribution from the two half-loops is 0 . Once the contours $C_{1}, C_{2}$ are closed, they can be deformed into the contour $C_{3}$. As $R \rightarrow \infty$, the integral becomes

$$
\frac{1}{2} \int_{C s} \frac{2 \sigma_{A}}{i \mu b} \frac{d z^{\prime}}{z^{\prime}}=\frac{1}{2}(-2 \pi i) \frac{2 \sigma_{A}}{i \mu b}=-\frac{2 \pi \sigma_{A}}{\mu b} .
$$

\subsection{2 equation (20)}

The integral in eqn. (20) is solved in much the same way as the integral in eqn. (15). However, in this instance, the pole does not lie on $L_{1}, L_{2}$. The final contours $C_{4}$ and $\mathrm{C}_{4}^{\prime}$ are shown in fig. 6-10. 

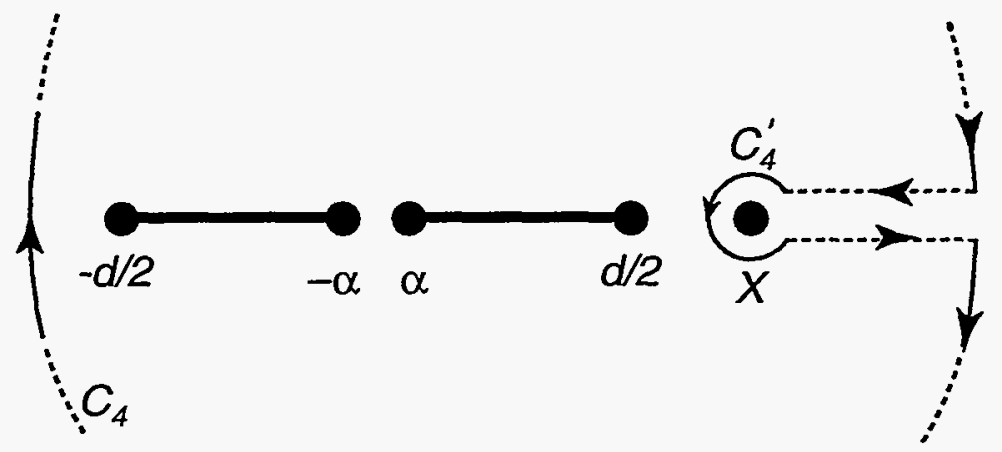

TRL-6342-6035(B)-O

Fig. 6-10. The integral in eqn. (20) is equal to $1 / 2 \times$ the contour integral over $C_{4}$ an infinite circle and $\mathrm{C}_{4}^{\prime}$, an infinitesimal circle at $z^{\prime}=x$.

The contribution from $C_{4}$ is just $\underline{2} 2 \pi$, and the contribution from $C_{4}^{\prime}$ is $i 2 \pi \times$ the residue at $x$. Both parts must be multiplied by $1 / 2$ since the contour integral over $C_{4}, C_{4}^{\prime}$ is twice the integral over $L_{1}, L_{2}$.

\subsection{3 equation (25)}

The integral in eqn. (25) can be performed by taking advantage of the fact that $\sigma_{p}(x)$ is known exactly and by taking advantage of the model's anti-symmetry about the y-axis in the complex plane. The integral over $L_{2}$ will be transformed into a contour integral over an infinite semi-circle (see fig. 6-11).

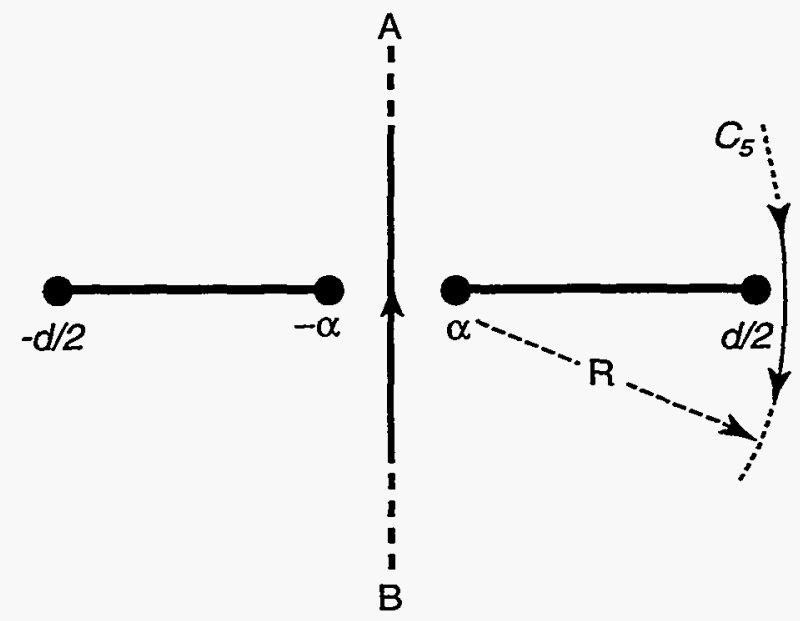

TRI-6342-6035(C)-0

Fig. 6-11. The first integral in eqn. (A7) over $L_{2}$ is $1 / 2 \times$ the integral over $C_{50}$ an infinite semicircle. The point $\mathrm{A}$ is located at $z=i R$, and the point $R$ is at $z=-i R$. Integrating from $\mathrm{B} \rightarrow \mathrm{A}$ over the imaginary axis gives eqn. (A11). Integrating over the arc (radius $R$ in the limit $R \rightarrow \infty$ ) gives eqn. (A12).

To perform this contour integral, $\sigma_{-}$must be known along the y-axis and along the arc with $R$ $\rightarrow \infty$.

Starting from eqn. (26), consider the analytic continuation of $\sigma_{-}$. Along the $y$-axis,

$$
\sigma_{-}(i y)=\frac{\mu b}{2 \pi} \int_{L_{1}} \frac{n\left(x^{\prime}\right) d x^{\prime}}{i y-x^{\prime}} .
$$


The real part of $\sigma_{-}(i y)$ is

$$
\mathrm{R}\left[\sigma_{-}(i y)\right]=\frac{\mu b}{2 \pi} \int_{L_{1}} \frac{-x^{\prime} n\left(x^{\prime}\right)}{x^{\prime 2}+y^{2}} d x^{\prime} .
$$

Notice that since $n\left(x^{\prime}\right)$ is odd for $x^{\prime} \in L_{1}, L_{2}$, the integrand of $\mathrm{R}\left[\sigma_{-}(i y)\right]$ is even in $x^{\prime} . \mathrm{R}\left[\sigma_{-}(i y)\right]$ can, therefore, be expressed as

$$
\mathrm{R}\left[\sigma_{-}(i y)\right]=\frac{\mu b}{2 \pi} \frac{1}{2}\left\{\int_{L_{1}}+\int_{L_{2}}\right\} \frac{-x^{\prime} n\left(x^{\prime}\right)}{x^{\prime 2}+y^{2}} d x^{\prime}=\frac{1}{2} \mathrm{R}\left[\sigma_{p}(i y)\right]=-\frac{\sigma_{A}}{2}+\frac{\sigma_{A}}{2} \sqrt{\frac{y^{2}+\alpha^{2}}{y^{2}+(d / 2)^{2}}} .
$$

The imaginary part,

$$
F\left[\sigma_{-}(i y)\right]=\frac{\mu b}{2 \pi} \frac{1}{2} \int_{L_{1}} \frac{-i y n\left(x^{\prime}\right)}{x^{\prime 2}+y^{2}} .
$$

cannot be solved so easily; however, it is sufficient to know that it is odd in $y$.

Finding $\sigma_{-}(z)$, when $z$ lies on the arc of radius $R \rightarrow \infty$ (fig. 6-11), is trivial. For large $|z|$,

$$
\sigma_{-}(z) \rightarrow \frac{\mu b}{2 \pi} \int_{L_{1}} \frac{n\left(x^{\prime}\right)}{z} d x^{\prime}=\frac{\mu b}{2 \pi} \frac{N}{z} .
$$

Although the formula for $N$ could be inserted here, it is quite messy, and in the end, all appearances of $N$ will cancel.

There is now sufficient information to find $\sigma_{\mathrm{b}}$. With foresight, the integral eqn. (25) is modified by adding 0 to the integrand in the form of $\left(\sigma_{\mathrm{A}} / 2-\sigma_{\mathrm{A}} / 2\right) n(x)$.

$$
\sigma_{b}=\int_{L_{1}} n(x)\left(\sigma_{-}(x)+\frac{\sigma_{A}}{2}\right) d x-\int_{L_{2}} n(x)\left(\frac{\sigma_{A}}{2}\right) d x
$$

The second integral is evaluated trivially as

$$
\frac{N \sigma_{A}}{2}
$$

The first integral in eqn. (A7) is transformed into the contour integral over $C_{5}$ (fig. 6-11). The integral over the $y$-axis is carried out

$$
\begin{gathered}
\frac{1}{2} \int_{-R}^{R} i d y n(i y)\left(\sigma_{-}(i y)+\frac{\sigma_{A}}{2}\right) \\
=\frac{1}{2} \int_{-4}^{R} i d y n(i y)\left(\mathrm{R}\left[\sigma_{-}(i y)\right]+\frac{\sigma_{A}}{2}\right)+\frac{1}{2} \int_{-R}^{R} i d y n(i y) \mathrm{F}\left[\sigma_{-}(i y)\right] .
\end{gathered}
$$

Since $n(i y)$ is even in $y$, and $\mathrm{F}\left[\sigma_{-}(i y)\right.$ is odd, the second integral in eqn. (Al0) vanishes leaving

$$
=-\frac{\sigma_{A}^{2}}{2 \mu b} \int_{-R}^{R} \frac{y^{2}+\alpha^{2}}{y^{2}+(d / 2)^{2}} d y=-\frac{\sigma_{A}^{2}}{2 \mu b}\left[2 R+\frac{2\left(\alpha^{2}-(d / 2)^{2}\right) \tan ^{-1}\left(\frac{R}{d / 2}\right)}{d / 2}\right]
$$

The integral over the arc in the limit $R \rightarrow \infty$ is

$$
\left.\frac{1}{2} \int_{A}^{B-2 \sigma A} \frac{-\mu b N}{i \mu b} \frac{1}{2 \pi}+\frac{\sigma_{A}}{2}\right) d z=-\frac{\sigma_{A} N}{2}+\frac{\sigma_{A}^{2} R}{\mu b} .
$$


Equation (25) is the sum of eqns. (A8), (A11) and (A12). 


\section{Unlimited Distribution:}

$1 \quad$ MS- 0188

10 MS- 1415

1 MS-1415

2 MS-1427

1 MS-9018

2 MS-0899

$1 \quad$ MS-0619

1 MS-0161
C. E. Meyers, 4523

T. A. Friedmann, 1112

W. B. Gauster, 1112

S. T. Picraux, 1100

Central Technical Files, 8940-2

Technical Library, 4916

Review and Approval Desk, 00111

Patent and Licensing Office, 11500 